\title{
Measurement of Neutrino-Nucleon Neutral-Current Elastic Scattering Cross-section at SciBooNE
}

\author{
Hideyuki Takei
}

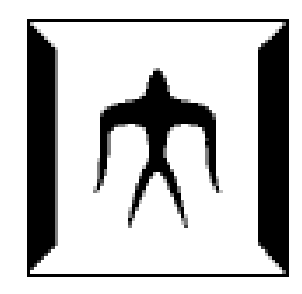

Thesis submitted to the Department of Physics in partial fulfillment of the requirements for the degree of

Doctor of Science at Tokyo Institute of Technology

February, 2009 


\begin{abstract}
In this thesis, results of neutrino-nucleon neutral current (NC) elastic scattering analysis are presented.

Neutrinos interact with other particles only with weak force. Measurement of cross-section for neutrino-nucleon reactions at various neutrino energy are important for the study of nucleon structure. It also provides data to be used for beam flux monitor in neutrino oscillation experiments.

The cross-section for neutrino-nucleon NC elastic scattering contains the axial vector form factor $G_{A}\left(Q^{2}\right)$ as well as electromagnetic form factors unlike electromagnetic interaction. $G_{A}$ is propotional to strange part of nucleon spin $(\Delta s)$ in $Q^{2} \rightarrow 0$ limit. Measurement of $\mathrm{NC}$ elastic cross-section with smaller $Q^{2}$ enables us to access $\Delta s$. NC elastic cross-sections of neutrinonucleon and antineutrino-nucleon were measured earlier by E734 experiment at Brookheaven National Laboratory (BNL) in 1987. In this experiment, cross-sections were measured in $Q^{2}>0.4 \mathrm{GeV}^{2}$ region. Result from this experiment was the only published data for $\mathrm{NC}$ elastic scattering cross-section published before our experiment.

SciBooNE is an experiment for the measurement of neutrino-nucleon scattering cross-secitons using Booster Neutrino Beam (BNB) at FNAL. BNB has energy peak at $0.7 \mathrm{GeV}$. In this energy region, $\mathrm{NC}$ elastic scattering, charged current elastic scattering, charged current pion production, and neutral current pion production are the major reaction branches.

SciBar, electromagnetic calorimeter, and Muon Range Detector are the detectors for SciBooNE. The SciBar consists of finely segmented scintillators and 14336 channels of PMTs. It has a capability to reconstruct particle track longer than $8 \mathrm{~cm}$ and separate proton from muons and pions using energy deposit information.

Signal of NC elastic scattering is a single proton track. In $\nu p \rightarrow \nu p$ process, the recoil proton is detected. On the other hand, most of $\nu n \rightarrow \nu n$ is invisible because there are only neutral particles in final state, but sometimes recoil neutron is scattered by proton and recoil proton is detected. Signal of this event is also single proton track. Event selection for the single proton track events using geometrical and $d E / d x$ information of reconstructed track is performed. After the event selection, $\mathrm{NC}$ elastic scattering data sample is obtained. They includes $\nu p \rightarrow \nu p$ and $\nu n \rightarrow \nu n$ is obtained. Absolute cross-section as a function of $Q^{2}$ is evaluated from this NC elastic scattering data sample.
\end{abstract}





\section{Contents}

\begin{tabular}{lll}
\hline 1 & Introduction & 13
\end{tabular}

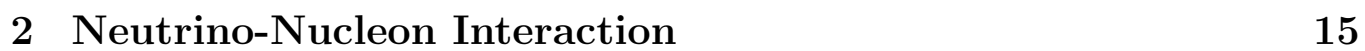

2.1 Neutrinos . . . . . . . . . . . . . . . . . . . . . . 15

2.1 .1 Charged Current Interactions . . . . . . . . . . . 16

2.1 .2 Neutral Current Interactions . . . . . . . . . . . . . . 16

2.2 Neutrino-Nucleon Neutral Current Elastic Scattering . . . . . 18

$2.2 .1 \quad$ Cross-Section . . . . . . . . . . . . . . . . 18

2.2 .2 Strangeness Component of Nucleon Spin . . . . . . . . 19

\begin{tabular}{|lll}
3 & Experimental Setup of SciBooNE & 27
\end{tabular}

3.1 Experimental Setup Overview . . . . . . . . . . . . . . . . 27

3.2 Booster Neutrino Beam . . . . . . . . . . . . . . . . . . 27

3.2 .1 Proton Beam . . . . . . . . . . . . . . . . 27

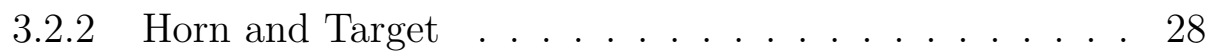

3.3 Detectors . . . . . . . . . . . . . . . . . . . . 30

3.3 .1 SciBar Detector . . . . . . . . . . . . . . . . . 30

3.3 .2 Electromagnetic Calorimeter . . . . . . . . . . . . . 33

3.3 .3 Muon Range Detector . . . . . . . . . . . . . . . . . . 34

3.3 .4 Triggers $\ldots \ldots \ldots \ldots \ldots \ldots$

3.4 Neutrino Events in the SciBar Detector . . . . . . . . . . . . . 36

3.5 Data Taking . . . . . . . . . . . . . . . . . . . . . . . . . 38

3.6 Beam Simulation . . . . . . . . . . . . . . . . . . . . . . . . . . . . . . . . 42

3.7 Neutrino Interaction Simulation . . . . . . . . . . . . . 45

$3.7 .1 \quad$ Elastic Scattering . . . . . . . . . . . . . . . . . . 45

3.7 .2 Resonant Single Meson Production . . . . . . . . . . . . . 45

3.7 .3 Coherent Pion Production . . . . . . . . . . . . . . . 47

3.7 .4 Nuclear Effects in Final State . . . . . . . . . . . . . 47

3.8 Detector Simulation . . . . . . . . . . . . . . . . . . . . . 50

3.9 Dirt Simulation . . . . . . . . . . . . . . . . . . . . . . . 52 
4 Analysis of Neutral Current Elastic Scattering 55

4.1 Track Reconstruction . . . . . . . . . . . . . . . . . . . . 55

4.2 Monte Carlo Normalization . . . . . . . . . . . . . . . . . . . 55

4.3 Event Selection . . . . . . . . . . . . . . . . . . . 58

4.3 .1 Geometrical Selection . . . . . . . . . . . . . . . . . 58

4.3 .2 Particle Identification . . . . . . . . . . . . . . . . . 60

4.4 Background Estimation . . . . . . . . . . . . . . . . . . . . . . 69

4.5 Event Selection Summary . . . . . . . . . . . . . . . . . 71

4.6 Detector Unfolding . . . . . . . . . . . . . . . . . . . . . . . . 72

4.7 Efficiency Correction . . . . . . . . . . . . . . . . . . . . . . . 74

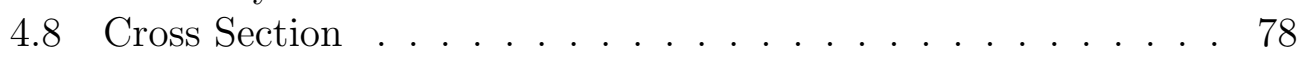

4.9 Systematic Uncertainty . . . . . . . . . . . . . . . . . . . . 80

4.9 .1 Neutrino Beam . . . . . . . . . . . . . . . . . 80

4.9 .2 Neutrino Interaction . . . . . . . . . . . . . . . . . . . . 81

$4.9 .3 \quad$ Detector Response . . . . . . . . . . . . . . . . . . . . 82

4.9 .4 Total Systematic Uncertainty . . . . . . . . . . . . . 83

4.10 Nuclear Effect Correction . . . . . . . . . . . . . . . . 86

$\begin{array}{lll}5 & \text { Result } & 91\end{array}$

\begin{tabular}{lll}
\hline 6 & Conclusion & 97
\end{tabular} 


\section{List of Figures}

2.1 Feynman diagrams of CC elastic and CC 1- $\pi$ production re-

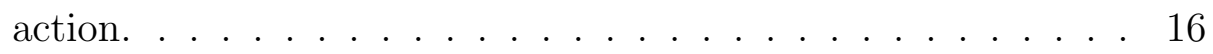

2.2 Cross-section for charged current interactions as a function of neutrino energy. Upper plot is for neutrino and lower plot is for antineutrino. . . . . . . . . . . . . . . . . . 17

2.3 Feynman diagrams of NC elastic and $\mathrm{NC} \pi^{0}$ production reaction. 18

2.4 Kinematics of $\nu p \rightarrow \nu p . \ldots \ldots \ldots$

2.5 Feynman diagram of electron-proton deep inelastic scattering. 22

2.6 The $\nu_{\mu}$ beam flux of E734 experiment. Dots show the measured $\nu_{\mu}$ flux. The solid curve is a MC beam flux prediction. . 23

2.7 The $\bar{\nu}_{\mu}$ beam flux of E734 experiment. Dots show the measured $\bar{\nu}_{\mu}$ flux. The solid curve is a MC beam flux prediction. . 23

2.8 (a) Differential cross-section for $\nu p \rightarrow \nu p$ (upper plots) and $\bar{\nu} p \rightarrow \bar{\nu} p$ (lower plots) measured by E734 experiment. (b) Ratio of the cross-section for $\nu p \rightarrow \nu p$ to the cross-section for $\bar{\nu} p \rightarrow \bar{\nu} p$. Solid lines and dashed lines show the result of fit. Error bars include statistic and $Q^{2}$ dependent systematic error. [1][10] . . . . . . . . . . . . . . . . . . . . . . . . . . . . . . . . . . . . 25

3.1 Experimental setup of SciBooNE. SciBooNE detector is located $100 \mathrm{~m}$ downstream of the proton target. MiniBooNE detector is located $440 \mathrm{~m}$ downstream of the SciBooNE detector. . . . . . . . . . . . . . . . . . . . 28

3.2 Air view of the Booster, target station, decay region and SciBooNE detector. . . . . . . . . . . . . . . . 29

3.3 SciBar, an electro-magnetic calorimeter (EC), and a muon range detector (MRD) are detectors for SciBooNE. They are located $100 \mathrm{~m}$ downstream of the proton target. . . . . . . . . 30 
3.4 The SciBar detector consists of 14,336 of the combination of plastic scintillator bar and wave-length shifting (WLS) fiber. Volume of the detector is $3 \times 3 \times 1.7 \mathrm{~m}^{3}$, weight is 15 tons. The Scintillator bars are arranged vertically and horizontally. Each WLS fiber is attached to one anode of a multi-anode PMT. . .

3.5 The WLS fibers are attached to the MAPMT by the cookie. The light injection module is located between the scintillators and the MAPMT. Each MAPMT is mounted on the front-end board. . . . . . . . . . . . . . . 33

3.6 The EC consists of $1 \mathrm{~mm}$ diameter scintillating fibers embedded in lead foil. The calorimeter is made of modules of dimensions $262 \times 8 \times 4 \mathrm{~cm}^{3}$. Each module is read out by two 1 inch Hamamatsu PMTs per side. . . . . . . . . . . .

3.7 The MRD is installed downstream of the EC. It has 12 iron plates with thickness of $5 \mathrm{~cm}$ and 13 plastic scintillator planes with thickness of $6 \mathrm{~mm}$. . . . . . . . . . . . .

3.8 A neutrino event candidate on online display. Vertical (left) and horizontal (right) readout of detectors are shown. . . . . . 36

3.9 Event displays of CCQE candidate. The area of dots is proportional to ADC channels. Boxes represent TDC hit information. Tracks penetrating the MRD are identified as a muon.

3.10 Event displays of CCQE candidate. The decay of the muon is tagged by multiple TDC hits. . . . . . . . . . . . . 37

$3.11 d E / d x$ distributions of muons (left plot) and protons (right plot). Protons are separated from muons with $\sim 90 \%$ efficiency. 37

3.12 CC $1 \pi$ coherent or $\nu n \rightarrow \mu^{-} n \pi^{+}$candidate. Muon and pion are visible. . . . . . . . . . . . . . . . . . . . . . 38

3.13 CC $1 \pi \nu p \rightarrow \mu^{-} p \pi^{+}$candidate. Muon, pion and proton are visible. . . . . . . . . . . . . . . . . . . . . . . 38

3.14 Candidate of neutral current elastic scattering. Signature of this event is single proton track. The proton is identified using

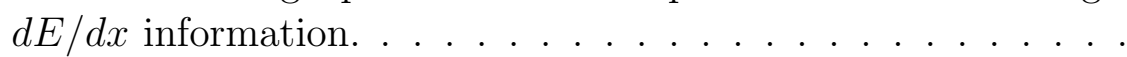

3.15 Candidate of neutral current $\pi^{0}$ production event. The $2 \gamma^{\prime}$ s from a $\pi^{0}$ decay produce electro-magnetic showers, which can be detected in the SciBar. . . . . . . . . . . . . . . . . . . . . 39

3.16 Accumulated number of POT (solid line) and number of POT which passed all the data quality cuts (dotted line). . . . . . 40

3.17 Number of CC event candidates normalized by POT. The flat shape for each mode and the same values for the two antineutrino mode periods shows that the SciBar took data stably throughout the beam time. . . . . . . . . . . . . 
3.18 Neutrino flux prediction at the SciBooNE detector as a function of neutrino energy $E_{\nu} . \ldots \ldots \ldots$. . . . . . . . 44

3.19 The $Q^{2}$ dependence of the differential cross-section for $\mathrm{NC}$ elastic scattering adopted in NEUT. Black line and red line shows differential cross-sections for neutrino-proton scattering and neutrino-neutron scattering respectively. . . . . . . . . . . 46

3.20 Probability of nucleon-nucleon interaction in ${ }^{16} \mathrm{O}$ after the neutrino interaction. No interaction (solid line), elastic scattering (dashed line), single $\pi$ production (dot-dot line), and two $\pi$ production (dash-dod line) are plotted. . . . . . . . . . 48

3.21 Mean deflection angle. It has a peak around 45 degrees. . . . . 49

3.22 Proton-proton total and elastic cross sections. . . . . . . . . 51

3.23 Proton-neutron total and elastic cross sections. . . . . . . . . . 51

3.24 Geometry of dirt for the dirt simulation. Gray areas indicate dirt volume. The geometry of $10 \times 10 \times 10 \mathrm{~m}^{3}$, taking detector origin (red point) as a center were considered. . . . . . . . . 53

3.25 Vertex distributions of dirt events from simulation. Only events which made one track contained in SciBar were selected. Geometry is large enough for NC elastic analysis. . . . . . . . . . 54

4.1 A candidate of neutral current elastic scattering from SciBooNE data. Signature of this event is single proton track. The proton is identified using stopping range and $d E / d x$ information. . . . . . . . . . . . . . . . 56

4.2 Flow chart of this analysis. . . . . . . . . . . . . 57

4.3 Total energy deposit of track-unrelated hits on top and side of SciBar. Data are compared to MC simulation. The threshold to select one track events is set to $30 \mathrm{MeV}$ for both top and side view. In these plots, dots with error bars is data and others are MC simulation. Red is NC elastic events, yellow is other interaction, and green is dirt events. . . . . . . . . . . 59

4.4 TDC hits from muon decay in top and side view. There are multiple TDC hits on both top and side view (case 1). . . . . 61

4.5 TDC hits from muon decay in top and side view. There are multiple TDC hits only on one view (case 2). . . . . . . . . 61

4.6 Time distribution of muon decay sample. Horizontal axis is the time interval between primary TDC hits and secondary TDC hits that matched on top and side view. . . . . . . . . 62 
4.7 Track length v.s. $d E / d x$. Red points represent protons and black points represent other particles such as $\mu^{ \pm}, \pi^{ \pm}$and $e^{ \pm}$. Line shows the cut for proton identification. Particles above the line are identified as protons. . . . . . . . . . . . . .

$4.8 d E / d x$ distributions for several different track lengths. The top left is $0-10 \mathrm{~cm}$, the bottom right is $70-80 \mathrm{~cm} . d E / d x$ is calculated from total energy deposit of the track divided by track length. The peak at large $d E / d x$ is from proton track while the peak at small $d E / d x$ is from other MIP particles. Data are compared to MC simulations. In these plots, dots with error bars is data and others are MC simulation. Red is NC elastic events, yellow is other interaction, and green is dirt

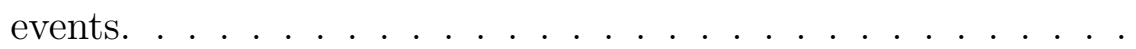

4.9 Angular distribution of NC elastic sample. $\theta$ is an angle of the track with respect to the $\mathrm{z}$ direction. Data are compared to MC simulation. Dots with error bars is data and others are MC simulation. Red is NC elastic events, yellow is other interaction, and green is dirt events. Right plot shows data/MC

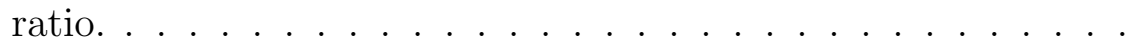

4.10 Length distribution (left figure) and data/MC ratio (right figure) of NC elastic sample. Data are compared to MC simulation. Dots with error bars is data and others are MC simulation. Red is NC elastic events, yellow is other interaction, and green is dirt events. Right plot shows data/MC ratio. . . 66

4.11 True proton kinetic energy v.s. true proton track length from MC simulation. A line is a result of fit. . . . . . . . . . . . 67

4.12 Distribution of proton kinetic energy. Proton kinetic energy is reconstructed from track length. Data are compared to MC simulation. . . . . . . . . . . . . .

4.13 Vertex position along $z$ axis v.s. track length of data (left), Monte-Carlo simulation in SciBar (middle) and dirt MonteCarlo simulation (right). SciBar is divided into 8 bins along $z$ direction to compare number of events at upstream part with that of downstream part. Fifth bin is taken as a reference and compared with first, second and third bins. . . . . . . . . . .

4.14 Track length distributions of (upstream bins)-(fifth bin). Data agree with Monte-Carlo simulation within statistic error. . . . 70

4.15 Proton kinetic energy distribution after background subtrac-

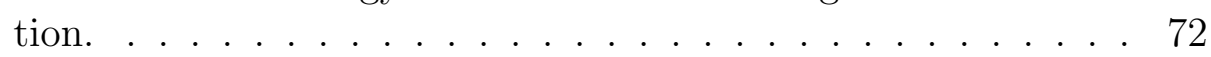


4.16 Proton kinetic energy reconstructed from track length using Monte-Carlo simulation v.s. proton kinetic energy reconstructed from measured track length using tracking algorithm (left figure). Both quantities are from MC simulation. From this relationship, unfolding matrix was made (right figure). $\ldots . .73$

4.17 Proton kinetic energy distribution after detector unfolding. Data are compared to MC simulation. . . . . . . . . 75

4.18 MC true proton kinetic energy v.s. detection efficiency. . . . . 76

4.19 Proton kinetic energy distribution after efficiency correction. Data are compared to MC simulation. . . . . . . . 77

4.20 Differential cross section as a function of proton kinetic energy. 79

4.21 Systematic uncertainty in the NC elastic cross section due to the uncertainty in the neutrino beam. . . . . . . . . . 81

4.22 The systematic uncertainty due to $M_{A}$ uncertainty. . . . . . . . 82

4.23 The systematic uncertainty due to the uncertainty in the detector response. . . . . . . . . . . . . . . . 84

4.24 Total systematic uncertainty in NC elastic scattering cross sec-

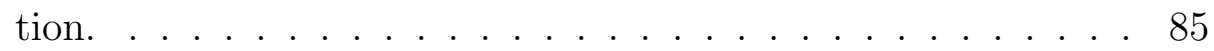

$4.25 Q^{2}$ reconstructed from proton kinetic energy (left) v.s. $Q^{2}$ calculated from the neutrino momentum using Monte-Carlo simulation (right). . . . . . . . . . . . . . . . . 86

$4.26 Q^{2}$ distribution after nuclear effect correction. Data are compared to MC simulation. . . . . . . . . . . 88

4.27 Differential cross section as a function of $Q^{2}$. Error bars include statistical error only. . . . . . . . . . . . . . . 89

5.1 Total systematic uncertainty in NC elastic scattering cross sec-

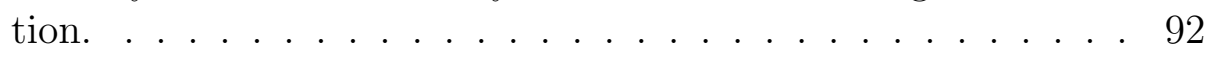

5.2 Differential cross section as a function of $Q^{2}$. Error bars include statistical error only. . . . . . . . . . . . . 93

5.3 Differential cross section after corrections. Cross section from data is compared with MC simulation. MC plot is from NC elastic sample and processed in the same way as data. Error bars include statistical error only. . . . . . . . . . . . . 94

5.4 Figure 5.4 shows differential cross section compared with the experimental data from BNL E734 and MiniBooNE experiments. Error bars of E734 and MiniBooNE data include statistical and systematic errors. BNL E734 data shows the cross section for $\nu p \rightarrow \nu p$ scattering while SciBooNE and MiniBooNE data show the cross section per nucleon $(\nu N \rightarrow \nu N)$. 95 



\section{List of Tables}

2.1 The values of the fitting parameters $G_{A}^{s}(0), F_{1}^{s}(0)$ and $F_{2}^{s}(0)$

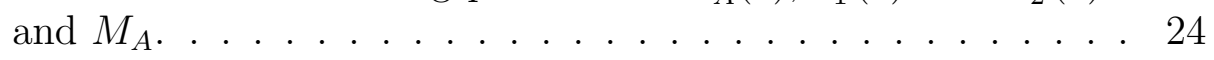

4.1 Particles which have roots in dirt events and make NC elastic scattering-like track in SciBar. . . . . . . . . . . . . . . 71

4.2 Fractions of the neutrino interactions in the dirt which make a proton track in SciBar. . . . . . . . . . . . . . . . 71

4.3 Event selection summary table of the NC elastic event selection. 71 



\section{Chapter 1}

\section{Introduction}

Neutrino is a particle introduced by Pauli in 1930. Electrons emitted in nuclear beta decay were expected to have a discrete energy for two-body decay, but they were discovered to have continuous energy spectrum. To maintain energy conservation, existence of neutral, light particle was suggested by Pauli. The particle is called "neutrino".

Neutrino is a particle with spin $1 / 2$. The neutrino is left-handed while anti-neutrino is right-handed. Neutrino interacts with nucleon only by weak interaction. The interaction is mediated either by a $\mathrm{W}$ boson or $\mathrm{Z}$ boson. Neutral Current (NC) interaction is an exchange of a Z boson while Charged Current (CC) interaction is an exchange of a $\mathrm{W}$ boson. Weak interaction only couples to left-handed particle. In other words, parity is violated.

Neutrino-nucleus scattering cross sections have been measured by several experiments. However, statistics of experimental data is low in energy region around $0.7 \mathrm{GeV}$.

In this energy region, several channels of neutrino interaction exist. In NC elastic scattering, there are one neutrino, one nucleon and nothing else in the final state $(\nu N \rightarrow \nu N)$. This channel is one of the main components of neutrino-nucleon interaction in the neutrino energy region around $0.7 \mathrm{GeV}$. In this energy region, the fractions of $\mathrm{NC}$ is $28.7 \%, \mathrm{CC}$ is $71.3 \%$. Fractions of $\mathrm{NC}$ elastic scattering is $17.6 \%, \mathrm{NC}$ pion production is $11.0 \%$, CC elastic scattering is $40.7 \%$ and $\mathrm{CC}$ pion production is $30.7 \%$.

Form factor describes a difference of cross sections between point-like target and target with actual size. Cross section for neutrino-nucleon NC elastic scattering contains electro-magnetic form factor and axial form factor. Each form factor includes strangeness part. Strangeness part of axial form factor $G_{A}$ is proportional to strangeness component of nucleon spin $(\Delta s)$ in the $Q^{2} \rightarrow 0$ limit. Quark spin contribution to the nucleon spin was measured by EMC experiment in 1988. It was only $12 \pm 9 \pm 14 \%$ though it was expected 
to be $100 \%$.

Cross section for NC elastic scattering was measured by E734 experiment at BNL in 1980's [1]. From the analysis of the data from this experiment, $\Delta s$ was evaluated to be between -0.21 and 0 . This experiment proved that $\Delta s$ can be measured by neutrino-nucleon scattering. However, the data from this experiment contains large systematic and statistic errors. One of the causes of large systematic error is that E734 was not able to measure cross section with $Q^{2}$ less than $0.4 \mathrm{GeV}^{2}$. New experiments are necessary to determine $\Delta s$ with a better accuracy. SciBooNE can measure cross section with $Q^{2}$ down to 0.2 $\mathrm{GeV}^{2}$ using fully-active, finely segmented tracking detector called "SciBar". It uses a high intensity neutrino beam from Booster Neutrino Beam at FNAL.

$\mathrm{CC}$ interactions are also measured at SciBooNE. Cross sections for $\mathrm{CC}$ interactions are independent of $\Delta s$. Taking ratio of cross sections of NC elastic events and CC events helps to cancel systematic errors in the beam profile.

Furthermore, it is important for neutrino experiments such as neutrino oscillation experiments to know cross sections of signal and background channels precisely.

SciBooNE can also provide neutrino flux information to neutrino oscillation experiment MiniBooNE which is on the same axis of the neutrino beam at FNAL. It improves sensitivity to the $\nu_{\mu}\left(\overline{\nu_{\mu}}\right)$ disappearance experiment [12]. 


\section{Chapter 2}

\section{Neutrino-Nucleon Interaction}

\section{$2.1 \quad$ Neutrinos}

Neutrinos are neutral, light particles with spin $1 / 2$. There are three flavors of neutrino: $\nu_{e}, \nu_{\mu}$, and $\nu_{\tau}$. They corresponds to the flavors of charged leptons, $e, \mu$ and $\tau$. Neutrinos interact only via $W^{ \pm}$and $Z^{0}$ bosons.

Neutral current (NC) is a process where a $Z^{0}$ boson is exchanged. Charged current $(\mathrm{CC})$ is a process where a $W^{ \pm}$boson is exchanged. Weak interaction has much less probability to take place compared to other interactions because the masses of $W^{ \pm}$and $Z^{0}$ are large $\left(80 \mathrm{GeV}\right.$ for $W^{ \pm}, 91 \mathrm{GeV}$ for $Z^{0}$ ) and needs a large amount of energy to produce.

Neutrinos have two state of handedness (chirality), left-handed and righthanded state. In the weak interaction, left-handed particles and right-handed anti-particles interact in different way. Interaction via $W^{+}$boson only couples to left-handed particle while interaction via $W^{-}$boson couples to righthanded anti-particle. In other words, parity is violated.

Helicity is the projection of the spin onto its momentum vector. It is written as

$$
h=\frac{(S, \vec{p})}{|\vec{p}|}
$$

where $S$ is the spin and $\vec{p}$ is the momentum of the particle. If the projection of the spin has the same direction as the momentum, helicity is positive. If the projection of the spin has opposite direction as the momentum, helicity is negative. For massless particles such as photon or gluon, helicity is Lorentz invariant because there is no frame that the projection of the spin is inverted. Their helicity is invariant and identical to chirality. For massive particles, helicity is not Lorentz invariant and distinguished from chirality. 
Only left-handed neutrinos and right-handed antineutrinos have been observed. It is considered that only left-handed neutrinos and right-handed antineutrinos exists. This fact is included in the Standard Model. Neutrinos is predicted to be massless in Standard Model. However, the experimental data from neutrino oscillation experiments showed that neutrinos have mass. The Standard Model must be extended to include the missing chiral states. Neutrinos with the missing chiral states are called "sterile" neutrinos and do not interact via $W^{ \pm}$or $Z^{0}$.

\subsubsection{Charged Current Interactions}

Charged current interactions are weak interactions via $W^{ \pm}$bosons. In neutrinonucleon scattering, neutrinos exchange charge with the nucleon and turn into charged leptons. Figure 2.1 shows Feynman diagrams of CC elastic and CC $1-\pi$ production reaction for $\nu_{\mu}$. In an experiment, $\mathrm{CC}$ interactions can be distinguished from $\mathrm{NC}$ interactions by finding charged leptons in the final state.

Cross-sections for CC interactions were measured by several experiment. However, in the neutrino energy region below $1 \mathrm{GeV}$, there are very low statistics for neutrino cross-section and no data for antineutrino cross-section (Figure 2.2). SciBooNE measures cross-sections in this energy region. It gives important information to neutrino experiments planning to use this energy region such as neutrino oscillation experiment T2K.
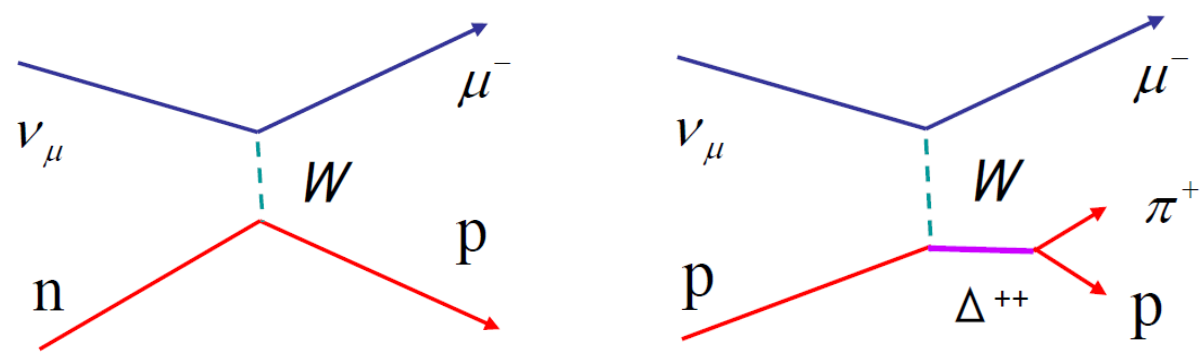

Figure 2.1: Feynman diagrams of CC elastic and CC 1- $\pi$ production reaction.

\subsubsection{Neutral Current Interactions}

Neutral current interaction was first observed in 1973 by the Gargamelle bubble chamber at CERN [3] 4]. They observed NC deep-inelastic scattering $\nu N \rightarrow \nu X$ and an elastic electron scattering event $\nu e^{-} \rightarrow \nu e^{-}$using $\nu_{\mu}$ and $\bar{\nu}_{\mu}$. 

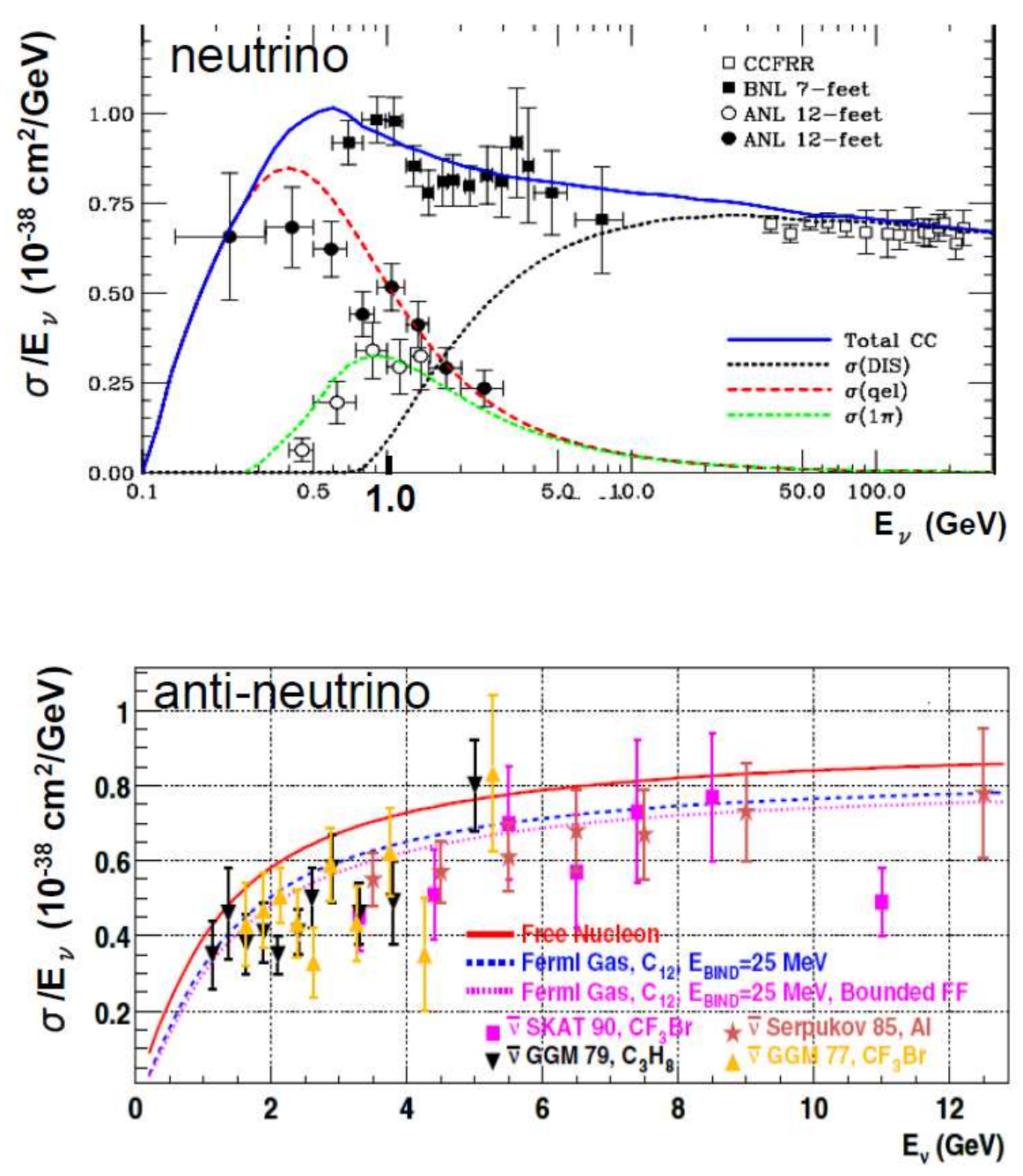

Figure 2.2: Cross-section for charged current interactions as a function of neutrino energy. Upper plot is for neutrino and lower plot is for antineutrino. 
Neutrino-nucleon NC elastic scattering was observed by the ColumbiaIllinois-Rockfeller (CIR) [5] and Harvard-Pennsylvania-Wisconsin (HPWB) [6] [7] collaborations at Brookhaven National Laboratory (BNL). They observed up to 100 events of $\nu p \rightarrow \nu p(\bar{\nu} p \rightarrow \bar{\nu} p)$. These experiments were able to set bounds on electroweak theory by constraining $\sin ^{2} \theta_{W}$. It was discovered by HPBW collaboration that the Lorentz structure of the neutral weak current is vector - axial-vector after taking more statistics.

There are several channels of neutrino-nucleus neutral current interaction such as elastic, pion production and deep inelastic scattering. Unlike CC events, there is no charged lepton in final state. NC events can be detected by finding hadrons or their decay particles in final state. Figure 2.3 shows Feynman diagrams of NC elastic and $\pi^{0}$ production via $\Delta$ resonance. Around $1 \mathrm{GeV}$ region of neutrino energy, the major fractions of interactions are elastic and pion production.
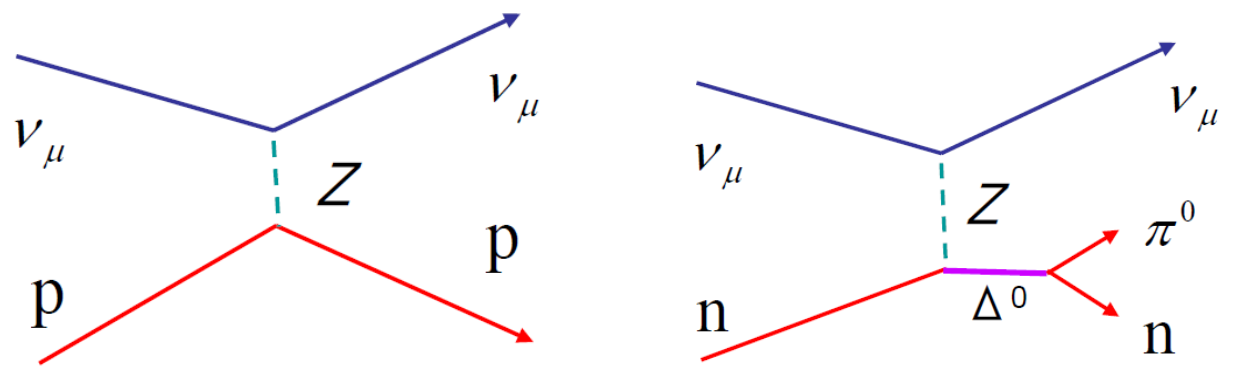

Figure 2.3: Feynman diagrams of $\mathrm{NC}$ elastic and $\mathrm{NC} \pi^{0}$ production reaction.

\subsection{Neutrino-Nucleon Neutral Current Elas- tic Scattering}

\subsubsection{Cross-Section}

The neutral weak current of the nucleon is written as

$$
J_{\mu}=<N\left(p^{\prime}\right)\left|F_{1}\left(Q^{2}\right) \gamma_{\mu}+F_{2}\left(Q^{2}\right) \sigma_{\mu \nu} q^{\nu}+G_{A}\left(Q^{2}\right) \gamma_{\mu} \gamma_{5}\right| N(p)>
$$

where $F_{1}\left(Q^{2}\right), F_{2}\left(Q^{2}\right)$, and $G_{A}\left(Q^{2}\right)$ are the nucleon form factors. The first and second terms are the vector part and the last term is the axial vector part.

The differential cross-section as a function of $Q^{2}$ is written as

$$
\frac{d \sigma}{d Q^{2}}=\frac{1}{64 \pi E_{\nu}^{2} M_{N}^{2}}|J|^{2}
$$


where the elements of the matrix $J$ is the neutral weak current of the nucleon from Equation 2.2. The differential cross-section using Llewellyn-Smith formalism [36] is written

$$
\frac{d \sigma}{d Q^{2}}=\frac{M_{N}^{2} G_{F}^{2}}{8 \pi E_{\nu}^{2}}\left[A\left(Q^{2}\right) \pm B\left(Q^{2}\right) \frac{s-u}{M_{N}^{2}}+C\left(Q^{2}\right) \frac{(s-u)^{2}}{M_{N}^{4}}\right]
$$

where the $G_{F}$ is the Fermi constant, $s-u=4 M_{N} E_{\nu}-Q^{2} . \pm$ in this equation is + for neutrinos and - for antineutrinos. $A\left(Q^{2}\right), B\left(Q^{2}\right)$ and $C\left(Q^{2}\right)$ contain the form factors.

$$
\begin{aligned}
A\left(Q^{2}\right)= & \frac{Q^{2}}{M_{N}^{2}}\left[G_{A}^{2}\left(1+\frac{Q^{2}}{4 M_{N}^{2}}\right)-F_{1}^{2}\left(1-\frac{Q^{2}}{4 M_{N}^{2}}\right)\right. \\
& \left.+F_{2}^{2}\left(1-\frac{Q^{2}}{4 M_{N}^{2}}\right) \frac{Q^{2}}{4 M_{N}^{2}}+F_{1} F_{2} \frac{Q^{2}}{M_{N}^{2}}\right] \\
B\left(Q^{2}\right)= & \frac{Q^{2}}{M_{N}^{2}} G_{A}\left(F_{1}+F_{2}\right) \\
C\left(Q^{2}\right)= & \frac{1}{4}\left[G_{A}^{2}+F_{1}^{2}+F_{2}^{2} \frac{Q^{2}}{4 M_{N}^{2}}\right]
\end{aligned}
$$

At low $Q^{2}, C\left(Q^{2}\right)$ term is dominant.

$Q^{2}$ can be written using recoil proton kinetic energy as

$$
Q^{2}=2 M_{p} T_{p}
$$

where $T_{p}$ is the kinetic energy of recoil proton. In SciBooNE experiment, proton track is distinguishable from other charged particles and kinetic energy of proton is reconstructed using track length. The incident neutrino energy is determined as

$$
E_{\nu}=\frac{M_{N}}{\cos \theta_{p}\left(1+2 M_{p} / T_{p}\right)^{1 / 2}-1}
$$

where $\theta_{p}$ is track angle of the recoil proton with respect to the beam direction. $Q^{2}$ and $E_{\nu}$ can be determined without measuring incident energy nor scattering angle of neutrino.

\subsubsection{Strangeness Component of Nucleon Spin}

The $Q^{2}$ dependence of the form factors is parameterized using a dipole form.

The $Q^{2}$ dependence of the axial form factor is given as

$$
G_{A}\left(Q^{2}\right)=\frac{1}{2} \frac{G_{A}(0)}{\left(1+Q^{2} / M_{A}^{2}\right)} \gamma_{3}+G_{A}^{s}\left(Q^{2}\right)
$$




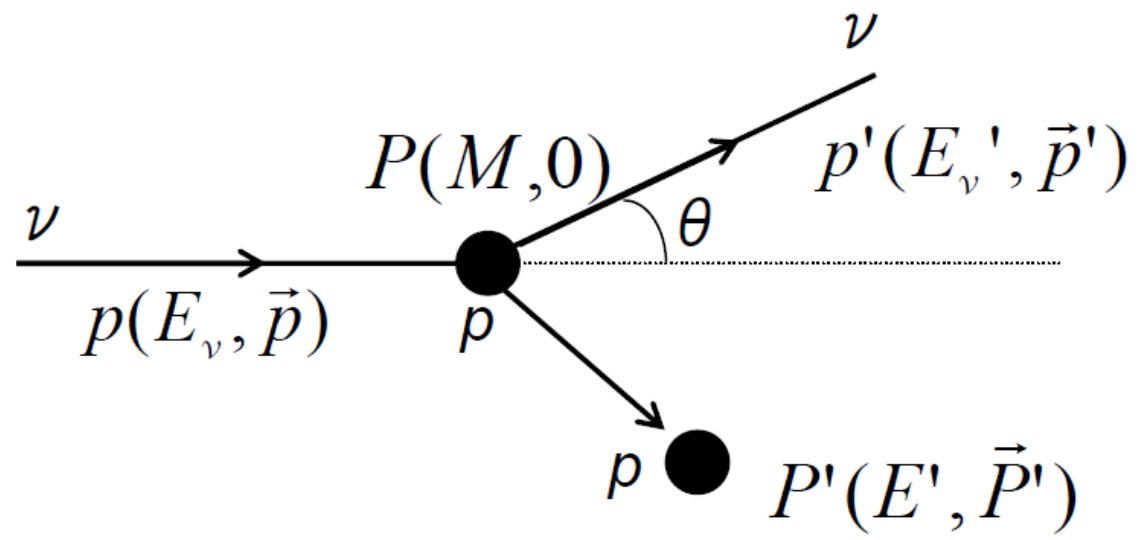

Figure 2.4: Kinematics of $\nu p \rightarrow \nu p$.

where $M_{A}$ is the dipole cutoff mass, $\gamma_{3}$ is +1 for proton and -1 for neutron. $G_{A}(0)$ is determined from beta decay.

When we consider strange quark contributions to the neutral weak nucleon current, additional form factors need to be introduced. Parameterized form factors are

$$
\begin{aligned}
F_{1}^{s}\left(Q^{2}\right) & =\frac{1}{6} \frac{-\gamma_{s}^{2} Q^{2}}{\left(1+Q^{2} / M_{1}^{2}\right)^{2}} \\
F_{2}^{s}\left(Q^{2}\right) & =\frac{\mu_{s}}{\left(1+Q^{2} / M_{2}^{2}\right)^{2}} \\
G_{A}^{s}\left(Q^{2}\right) & =\frac{1}{2} \frac{\Delta s}{\left(1+Q^{2} / M_{A}^{2}\right)^{2}}
\end{aligned}
$$

where $M_{1}$ and $M_{2}$ are the relevant masses of the strange vector form factors, $\gamma_{s}$ is the strange radius of the nucleon (analogous to the nucleon charge radius), $\mu_{s}$ is the strange anomalous magnetic moment of the nucleon, and $\Delta s$ is the strange component of nucleon spin.

\section{Measurement of $\Delta s$ using electron beams}

$\Delta s$ can be measured using electron-nucleon deep inelastic scattering (DIS). Figure 2.5 shows a Feynman diagram of electron-proton deep inelastic scattering. $Q^{2}$ of this interaction is written as

$$
\begin{aligned}
Q^{2} & =-q^{2} \\
& =-\left(k-k^{\prime}\right)^{2}
\end{aligned}
$$




$$
=4 E E^{\prime} \sin ^{2} \frac{\theta}{2}
$$

where $k=(E, \vec{k})$ and $k^{\prime}=\left(E^{\prime}, \overrightarrow{k^{\prime}}\right)$ are the momentum of electron before and after scattering, $P=(M, 0)$ is the momentum of target proton, and $\theta$ is the scattering angle. Invariant mass $W$ is

$$
\begin{aligned}
W^{2} & =(P+q)^{2} \\
& =M^{2}+2 P q+q^{2} \\
& =M-2+2 M \nu-Q^{2}
\end{aligned}
$$

where $\nu$ is the energy transfered from electron to proton by photon. $\nu$ is written

$$
\nu=\frac{P q}{M}
$$

Bjorken scaling parameter $x$ is defined as

$$
x=\frac{Q^{2}}{2 P q} .
$$

For DIS, the value of $x$ is $0<x<1$. Spatial resolution increases as $Q^{2}$ become larger and electrons are scattered by point-like particles in the nucleon as was confirmed by past experiments [9]. This point-like particles were called partons that are known as quarks today. $x$ represents a fraction of the momentum of a quark inside nucleon at the limit of $Q^{2} \rightarrow \infty$. In DIS interaction, a single quark directly interacts with a virtual photon. It does not interacts with any other quarks. Probability of electron-quark interaction with $x$ is represented by quark distribution function. Sum of quark distribution functions of strange quark $s(x)$ and its antiparticle $\bar{s}(x)$ is written as $\Delta S(x)=s(x)+\bar{s}(x)$. Strange quark contribution to the nucleon spin is obtained by performing integration of $\Delta S(x)$ from $x=0$ to $x=1$.

\section{Measurement of $\Delta s$ using neutrino beams}

$\Delta s$ can be measured using $\nu N \rightarrow \nu N$ interaction. This interaction is neutral current elastic scattering.

Neutrino-nucleon and antineutrino-nucleon elastic scattering cross-section were measured by BNL E734 experiment [1]. The E734 experiment used the neutrino beam with the peak energy of $0.6 \mathrm{GeV}$, the mean energy of $1.2 \mathrm{GeV}$. $79 \%$ of protons in the detector is bound in carbon and aluminum. $21 \%$ of protons are free. Figure2.6 and Figure2.7 shows the $\nu_{\mu}$ and $\bar{\nu}_{\mu}$ beam flux of E734 experiment. 


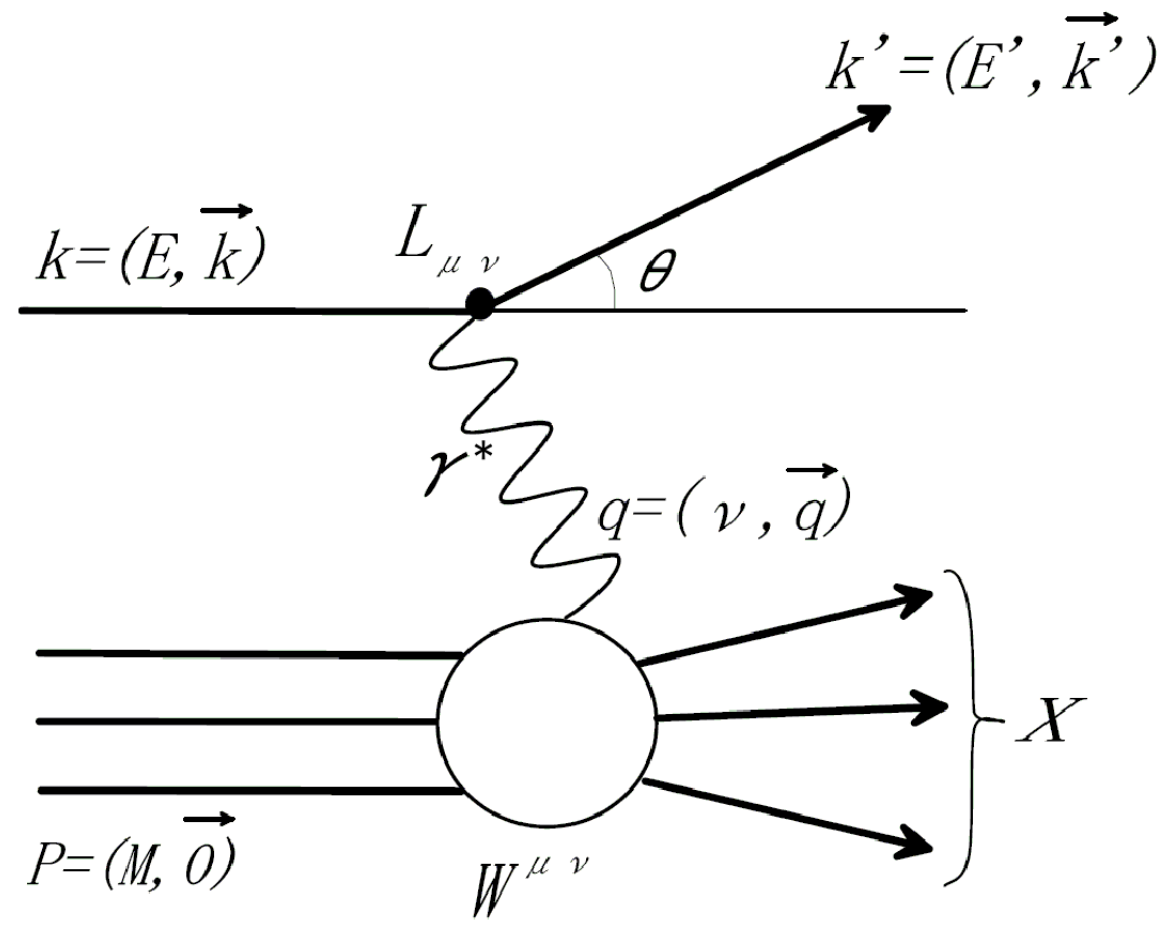

Figure 2.5: Feynman diagram of electron-proton deep inelastic scattering. 


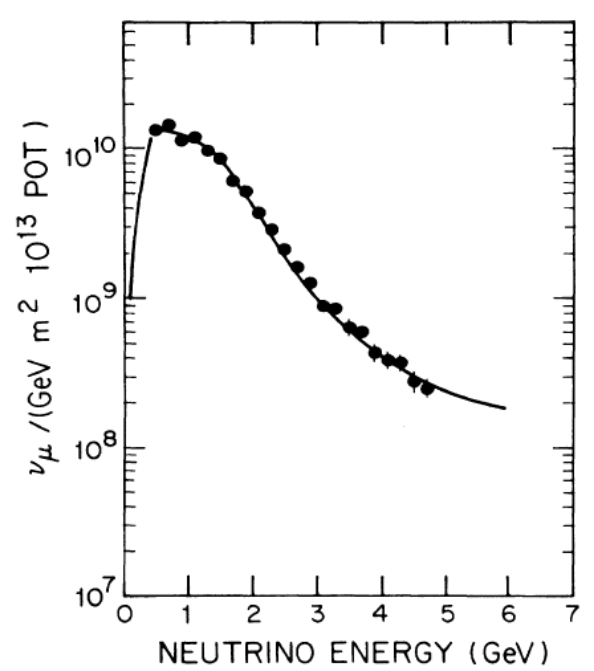

Figure 2.6: The $\nu_{\mu}$ beam flux of E734 experiment. Dots show the measured $\nu_{\mu}$ flux. The solid curve is a MC beam flux prediction.

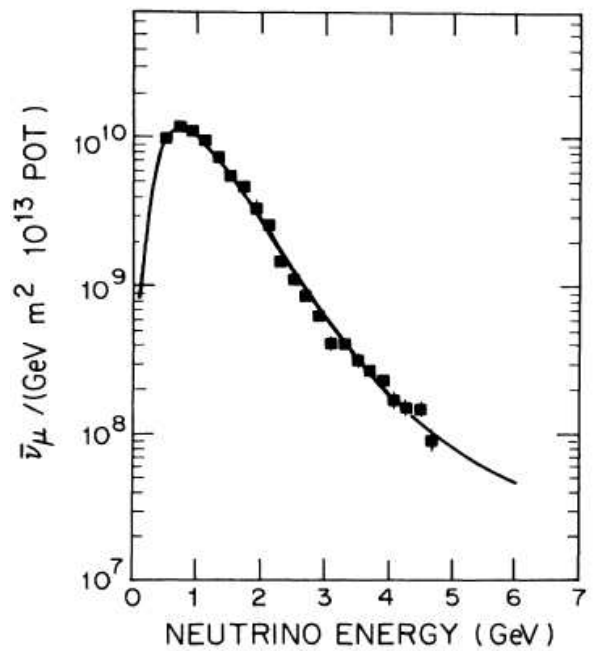

Figure 2.7: The $\bar{\nu}_{\mu}$ beam flux of E734 experiment. Dots show the measured $\bar{\nu}_{\mu}$ flux. The solid curve is a MC beam flux prediction. 


\begin{tabular}{|c|c|c|c|c|c|}
\hline Fit & $G_{A}^{s}(0)$ & $F_{1}^{s}(0)$ & $F_{2}^{s}(0)$ & $M_{A}$ & $\chi / N_{D O F}$ \\
\hline 1 & 0 & 0 & 0 & $1.086 \pm 0.015$ & $14.12 / 14$ \\
\hline 2 & $-0.15 \pm 0.07$ & 0 & 0 & $1.049 \pm 0.019$ & $9.73 / 13$ \\
\hline 3 & $-0.13 \pm 0.09$ & $0.49 \pm 0.70$ & $-0.39 \pm 0.70$ & $1.049 \pm 0.023$ & $9.28 / 11$ \\
\hline 4 & $-0.21 \pm 0.10$ & $0.53 \pm 0.70$ & $-0.40 \pm 0.72$ & $1.012 \pm 0.032$ & $8.13 / 11$ \\
\hline
\end{tabular}

Table 2.1: The values of the fitting parameters $G_{A}^{s}(0), F_{1}^{s}(0)$ and $F_{2}^{s}(0)$ and $M_{A}$.

Re-analysis of the E734 data was performed [10]. Figure 2.8 (a) shows the differential cross-section for $\nu p \rightarrow \nu p$ (upper plots) and $\bar{\nu} p \rightarrow \bar{\nu} p$ (lower plots) measured by E734 experiment. Figure 2.8 (b) shows the ratio of the cross-section for $\nu p \rightarrow \nu p$ to the cross-section for $\bar{\nu} p \rightarrow \bar{\nu} p$. Solid lines and dashed lines show the result of fit. Error bars include statistic and $Q^{2}$ dependent systematic error. From this data, form factors $G_{A}^{s}(0), F_{1}^{s}(0)$ and $F_{2}^{s}(0)$ are determined by fitting. The $\chi^{2}$ is defined as

$$
\begin{aligned}
\chi^{2}= & \sum_{\nu} \frac{\left(\sigma_{i}^{f}-\sigma_{i}^{d} / s_{\nu}\right)^{2}}{\delta \sigma_{i}^{2}}+\sum_{\bar{\nu}} \frac{\left(\sigma_{i}^{f}-\sigma_{i}^{d} / s_{\bar{\nu}}\right)}{\delta \sigma_{i}^{2}} \\
& +\frac{\left(1-s_{\nu} s_{\bar{\nu}}\right)^{2}}{0.153^{2}}+\frac{\left(1-s_{\nu} / s_{\bar{\nu}}\right)^{2}}{0.153^{2}}+\frac{\left(M_{A}-1.061\right)^{2}}{0.026^{2}}
\end{aligned}
$$

where $\sum_{\nu}$ and $\sum_{\bar{\nu}}$ are summations over all measurement points. $\sigma_{i}^{f}$ is the differential cross section calculated from the form factors considered as parameters, $\sigma_{i}^{d}$ is the measured differential cross section. $s_{\nu}$ and $s_{\bar{\nu}}$ are scale factors of neutrino and antineutrino data. $\delta \sigma_{i}$ is statistical and $Q^{2}$ dependent systematic error, 0.153 and 0.108 are $Q^{2}$ independent systematic error. $1.061 \pm 0.026 \mathrm{GeV}$ is axial dipole mass. Fitting was performed changing $G_{A}^{s}(0), F_{1}^{s}(0)$ and $F_{2}^{s}(0)$. The result of the fitting is shown in Table 2.1. Solid line in Figure 2.8 is the result of fit 1 and dashed line is the result of fit 4 . From this fitting, $\Delta s$ turned out to have a value from -0.21 to 0 .

This experiment showed that $\Delta s$ can be measured using neutrino-nucleon $\mathrm{NC}$ elastic interaction. However, more experiments with new techniques are needed for more precise measurements since the E734 expeirment had large systematic error. 


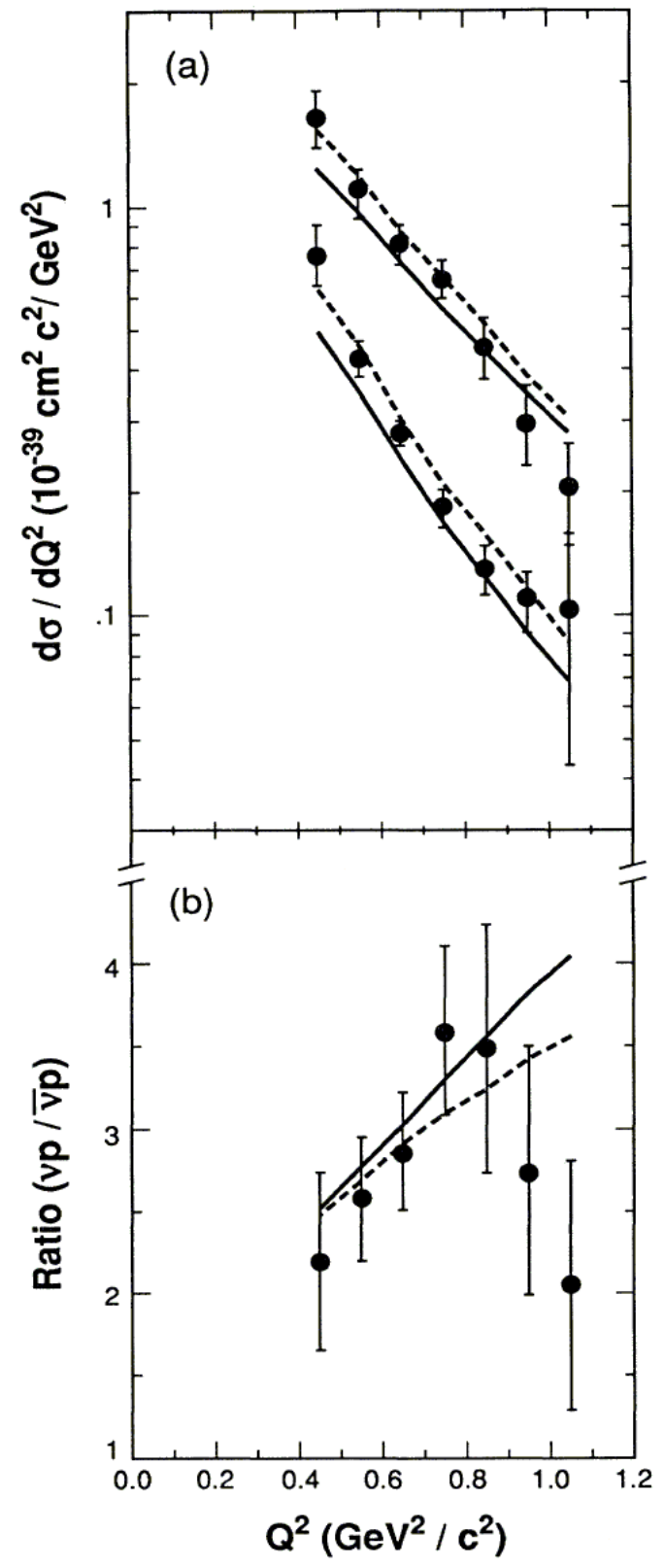

Figure 2.8: (a) Differential cross-section for $\nu p \rightarrow \nu p$ (upper plots) and $\bar{\nu} p \rightarrow \bar{\nu} p$ (lower plots) measured by E734 experiment. (b) Ratio of the crosssection for $\nu p \rightarrow \nu p$ to the cross-section for $\bar{\nu} p \rightarrow \bar{\nu} p$. Solid lines and dashed lines show the result of fit. Error bars include statistic and $Q^{2}$ dependent systematic error. [1] [10] 



\section{Chapter 3}

\section{Experimental Setup of SciBooNE}

\subsection{Experimental Setup Overview}

SciBooNE uses the FNAL Booster Neutrino Beam (BNB) with a peak energy of $0.7 \mathrm{GeV}$. Protons with $8 \mathrm{GeV}$ from the Booster hit a beryllium target and produces mesons, i.e. pions and kaons. The target is surrounded by a magnetic focusing horn. The produced mesons are focussed, and decay inside a $50 \mathrm{~m}$ long decay volume: $\pi^{ \pm} \rightarrow \mu^{ \pm}+\nu(\bar{\nu}), K^{ \pm} \rightarrow \mu^{ \pm}+\nu(\bar{\nu})$. The polarity of the magnetic focusing horn can be changed to select the neutrino or antineutrino mode which enables us to study both neutrino and antineutrino cross-sections. SciBar, an electro-magnetic calorimeter (EC) [16], and a muon range detector (MRD) [17] are detectors for SciBooNE. They are located $100 \mathrm{~m}$ downstream of the proton target. MiniBooNE detector is located $440 \mathrm{~m}$ downstream of the SciBooNE detector. Experimental setup of SciBooNE is shown in Figure 3.1. Air view of the Booster, target station, decay region and SciBooNE detector are shown in Figure 3.2

\subsection{Booster Neutrino Beam}

\subsubsection{Proton Beam}

$8 \mathrm{GeV}$ protons from the Booster as the primary beam are provided to the target station. The proton beam has approximately $4 \times 10^{12}$ protons per $\sim 1.6$ micro second beam batch at a maximum rate of $5 \mathrm{~Hz}$. The timing structure within each beam batch has 81 bunches, each bunch is approximately $6 \mathrm{~ns}$ wide, separated by $\sim 19 \mathrm{~ns}$. 


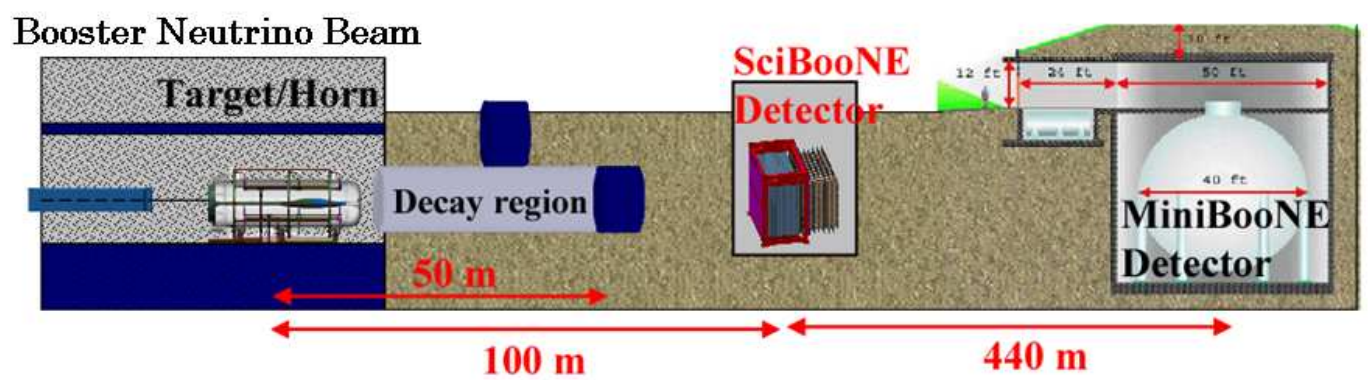

Figure 3.1: Experimental setup of SciBooNE. SciBooNE detector is located $100 \mathrm{~m}$ downstream of the proton target. MiniBooNE detector is located 440 $m$ downstream of the SciBooNE detector.

Trajectories and positions of the proton beam are measured on a pulseby-pulse basis. The beam position monitors located near the target measure the beam alignment and divergence. The beam position and angle are automatically adjusted to keep the proton beam centered on the target. The beam profile monitors measure beam focusing on the target. The number of protons delivered to the target is measured for each proton batch with two toroids located near the target along the beam line.

\subsubsection{Horn and Target}

The proton target is located in a target station. The target is $71 \mathrm{~cm}$ long, $1 \mathrm{~cm}$ diameter beryllium. Primary protons hit the target and secondary mesons (pions and kaons) are produced.

The target is enclosed in the magnetic focusing horn. The horn makes coaxial magnetic field in the direction of the beam to focus the secondary mesons produced on the target. The horn current pulse is approximately $174 \mathrm{kA}, 143 \mu \mathrm{s}$ long, synchronized to each beam spill. The horn can change the polarity of magnetic field to focus positive charged particles and defocus negative charged particles, and vice versa.

The secondary mesons from the target decay into neutrinos in the decay region. The decay region is $50 \mathrm{~m}$ long and its radius is $90 \mathrm{~cm}$. The cylindrical volume is filled with air at atmospheric pressure. A beam absorber is located at the end of the decay region. It stops the hadronic and muonic component of the beam. 


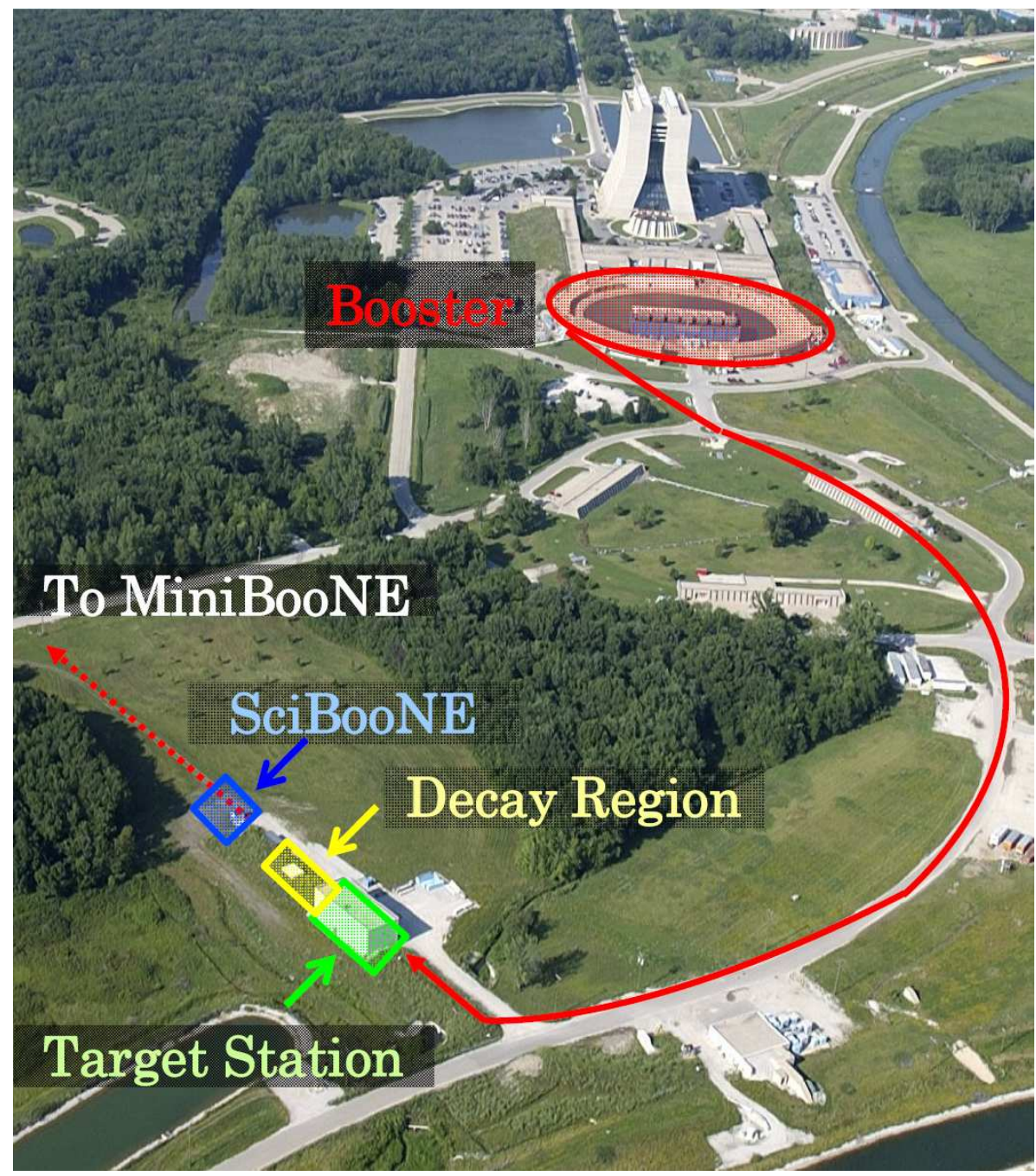

Figure 3.2: Air view of the Booster, target station, decay region and SciBooNE detector. 


\subsection{Detectors}

SciBar, an electro-magnetic calorimeter (EC) [16], and a muon range detector (MRD) [17 are detectors for SciBooNE (Figure 3.3). They are located 100 $\mathrm{m}$ downstream of the proton target. A right-handed Cartesian coordinate system is used. $z$ axis is the beam direction and $y$ axis is the vertical upward direction. The origin of the coordinate system is defined as the center of SciBar in $x y$ plane and the most upstream edge of SciBar in $z$ direction.

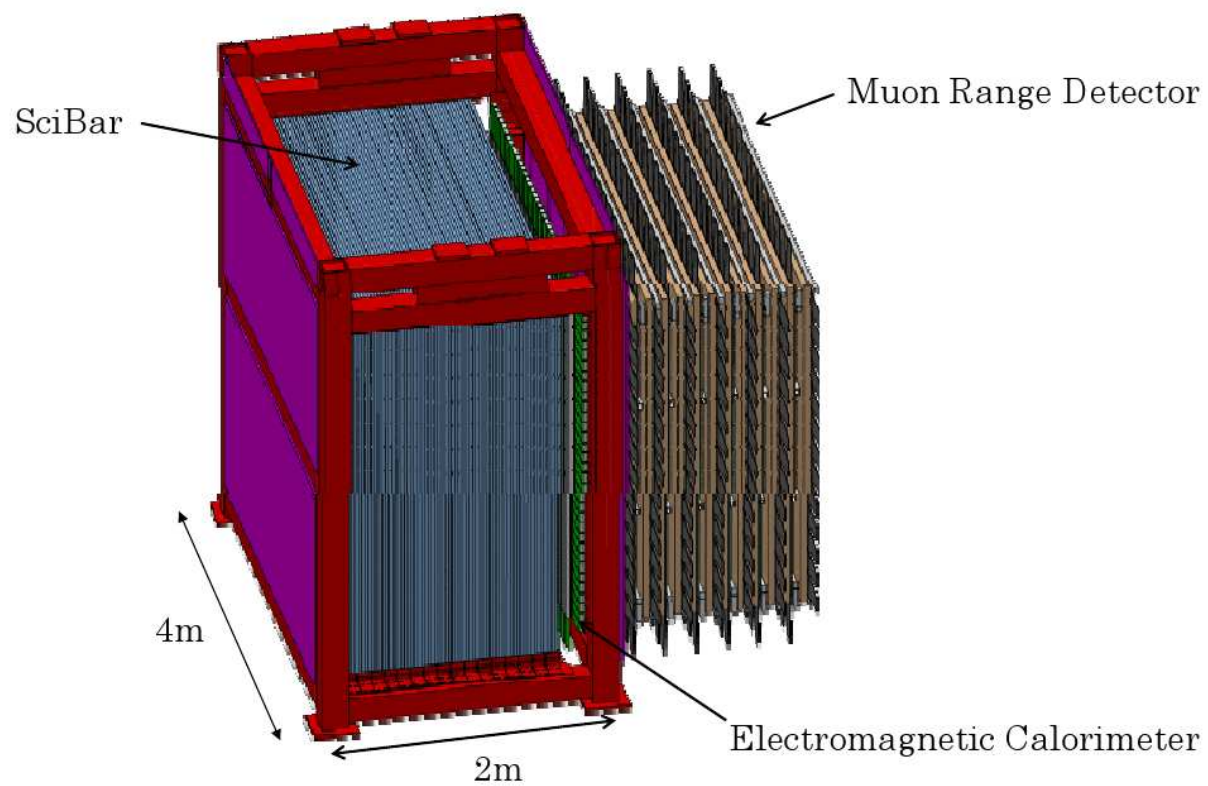

Figure 3.3: SciBar, an electro-magnetic calorimeter (EC), and a muon range detector (MRD) are detectors for SciBooNE. They are located $100 \mathrm{~m}$ downstream of the proton target.

\subsubsection{SciBar Detector}

The SciBar detector [15] is a fully-active neutrino detector with finely segmented structure. Carbon and hydrogen in the plastic scintillators are the target nuclei. Its volume is $3 \times 3 \times 1.7 \mathrm{~m}^{3}$ and weight is 15 tons. The SciBar detector consists of 14,336 plastic scintillator bars (Figure 3.4). Dimensions 
of each plastic scintillator bar are $1.3 \times 2.5 \times 300 \mathrm{~cm}^{3}$. The SciBar detector consists of 64 layers arranged perpendicular to the beam direction. Each layer has a vertical plane and a horizontal plane. One plane consists of 112 bars of plastic scintillator arranged vertically or horizontally. The scintillator bars are made of polystyrene, infused with PPO (1\%) and POPOP $(0.03 \%)$ with $0.25 \mathrm{~mm}$ thickness $\mathrm{TiO}_{2}$ coating. The emission wave length is $420 \mathrm{~nm}$ at peak position. One wave length shifting (WLS) fiber is inserted in each plastic scintillator bar. The diameter of the WLS fiber is $1.5 \mathrm{~mm}$. The attenuation length of each WLS fiber is measured, and is about $350 \mathrm{~cm}$ on average. The scintillation light from the plastic scintillators is sent to a 64-channel multi-anode photo-multiplier tube (MAPMT) through the WLS fibers. In total, 224 of MAPMT (H8804 made by Hamamatsu Photonics K.K.) are used in the SciBar detector. Each has 64 anodes with $2 \times 2 \mathrm{~mm}^{2}$ area. The typical gain is $6 \times 10^{5}$ with a linear response up to $\sim 200$ photoelectrons (p.e.); the gain uniformity over all channels is $20 \%$ in RMS. The fibers and the MAPMT are connected using a dedicated jig, called a "cookie" (Figure3.5) to align the fibers to the anodes. There is cross-talk mainly caused by light entering adjacent channels on the surface of the MAPMT. The average level of the cross-talk from an adjacent channel is measured to be $3.5 \%$.

The gains of all the SciBar channels are monitored using a gain monitoring system which consists of LEDs, clear fibers, and light injection modules. The light from four LEDs is sent via clear fibers to each light injection module located between the plastic scintillators and every MAPMT. The light injection modules are designed to uniformly distribute light from the clear fibers to each WLS fiber. PIN-photo diodes are located near each LED to monitor the LED luminosity. The gain and pedestal data for each MAPMT channel are taken between every beam spill to monitor the detector stability.

Light yield and timing resolution of the SciBar detector are evaluated using cosmic-ray muons. Light yield is $\sim 20$ p.e. $/ 1.3 \mathrm{~cm}$ for minimum ionizing particles at the detector edge close to the MAPMT. Timing resolution is $\sim 1.6$ ns. The number of dead channels of the SciBar detector is one out of 14,336 .

Each MAPMT is mounted on a front-end board as shown in Figure 3.5. Each front-end board has two sets of Application Specific Integrated Circuit (ASIC), called "VA/TA". The VA is an ASIC with a preamplifier, a shaper and a multiplexer which serialize charge from 32 channels of the MAPMT to a single output. The TA is an ASIC which provides inclusive timing information for the 32 channels. The front-end boards process signals from the MAPMTs, and send them to VME-9U backend electronics boards. They process the charge and timing information from the MAPMT with 12-bit flash ADCs and 64-ch multi-hit TDCs.

Charged particles whose track length exceeds $8 \mathrm{~cm}$ inside the detector are 


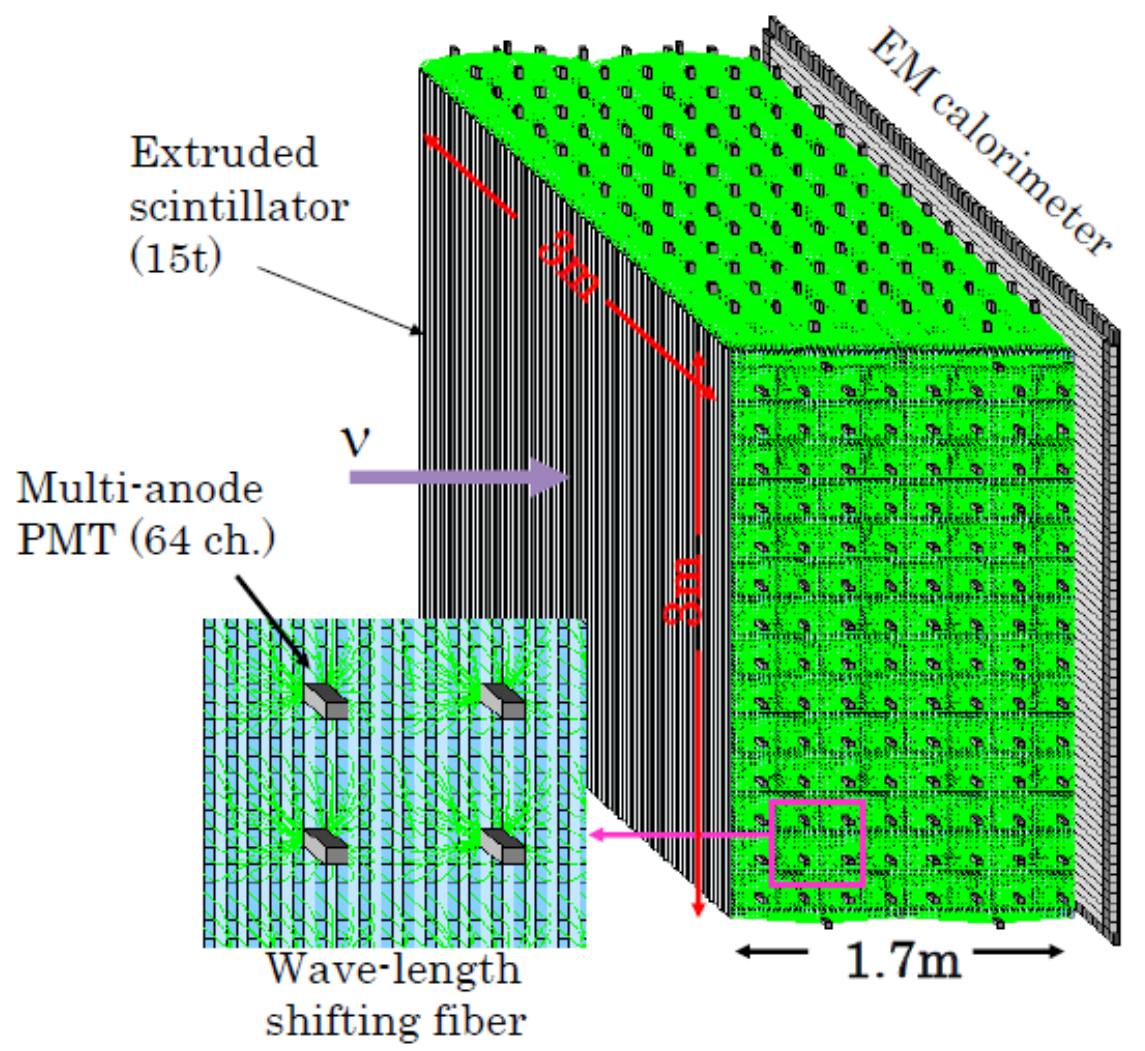

Figure 3.4: The SciBar detector consists of 14,336 of the combination of plastic scintillator bar and wave-length shifting (WLS) fiber. Volume of the detector is $3 \times 3 \times 1.7 \mathrm{~m}^{3}$, weight is 15 tons. The Scintillator bars are arranged vertically and horizontally. Each WLS fiber is attached to one anode of a multi-anode PMT. 
reconstructed with $99 \%$ efficiency.

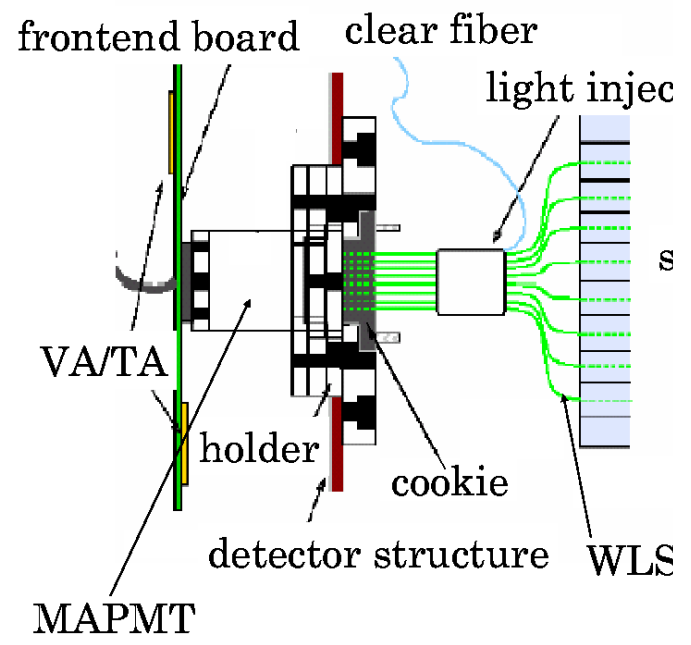

Figure 3.5: The WLS fibers are attached to the MAPMT by the cookie. The light injection module is located between the scintillators and the MAPMT. Each MAPMT is mounted on the front-end board.

\subsubsection{Electromagnetic Calorimeter}

The Electromagnetic Calorimeter called Electron Catcher (EC) is located downstream of SciBar. The EC is designed to measure the electron neutrino $\left(\nu_{e}\right)$ contamination in the beam and to tag $\gamma^{\prime}$ s from $\pi^{0}$ decay. The EC consists of $1 \mathrm{~mm}$ diameter scintillating fibers embedded in lead foil (Figure 3.6). The calorimeter is made of modules of dimensions $262 \times 8 \times 4 \mathrm{~cm}^{3}$. Each module is read out by two 1 inch Hamamatsu PMTs per side. Total number of PMTs is 256. The EC modules were built for the CHORUS neutrino experiment at CERN [18] and later used in HARP and then K2K. The modules construct one vertical and one horizontal plane, and each plane has 32 modules. The EC has a thickness of 11 radiation length along the beam direction. An active area of the plane is $2.7 \times 2.6 \mathrm{~m}^{2}$. A minimum ionizing particle with a minimal path length deposits approximately $85 \mathrm{MeV}$. The energy resolution for electrons was measured to be $14 \% / \sqrt{E(\mathrm{GeV})}$ using a test beam. 


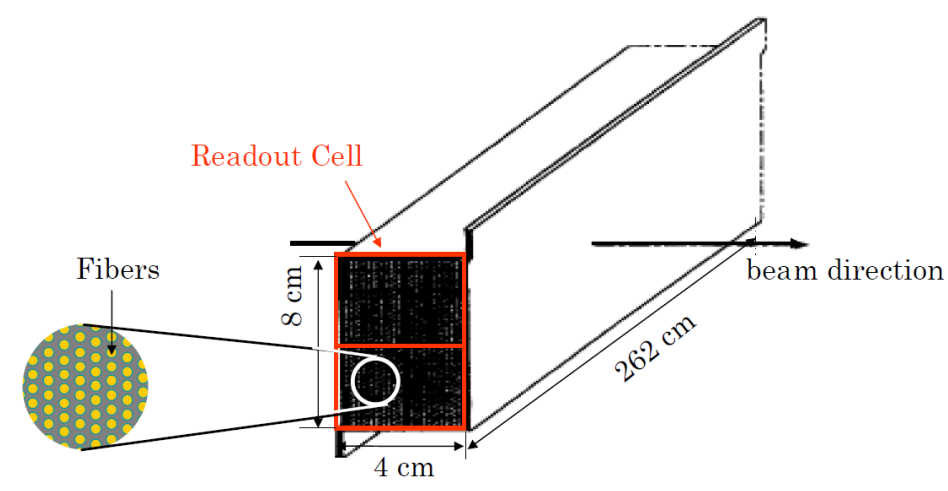

Figure 3.6: The EC consists of $1 \mathrm{~mm}$ diameter scintillating fibers embedded in lead foil. The calorimeter is made of modules of dimensions $262 \times 8 \times 4$ $\mathrm{cm}^{3}$. Each module is read out by two 1 inch Hamamatsu PMTs per side.

\subsubsection{Muon Range Detector}

The MRD (figure 3.7) is installed downstream of the EC. The MRD is designed to measure the momentum of muons. It has 12 iron plates with thickness of $5 \mathrm{~cm}$ and 13 plastic scintillator planes with thickness of $6 \mathrm{~mm}$. Iron plates are sandwiched between scintillator planes. Scintillator counters are aligned altering horizontal and vertical planes with 2 inch PMTs. Total number of PMTs are 362. Iron plates cover areas of $274 \times 305 \mathrm{~cm}^{2}$. The MRD measures the momentum of muons up to $1.2 \mathrm{GeV} / \mathrm{c}$ using the observed muon range. Hit finding efficiency was continuously monitored using cosmic ray data taken between beam spills. The average hit finding efficiency is $99 \%$.

\subsubsection{Triggers}

There are two triggers, for neutrino data taking (beam trigger) and for detector calibration data (off-beam trigger). They are taken in every beam cycle. One cycle is about 2 seconds. Beam trigger is a fast timing signal from the extraction magnet on BNB. Off-beam trigger is set after the beam trigger. Each detector takes pedestal and cosmic ray data. SciBar and the EC use a common cosmic ray trigger generated using fast signals from the TA. The MRD has independent self-generated cosmic ray trigger. SciBar also takes LED data for monitoring relative gain of all channels.

Events taken by beam trigger and off-beam trigger is checked by shifter once per hour using online event display. Neutrino events can be seen occa- 


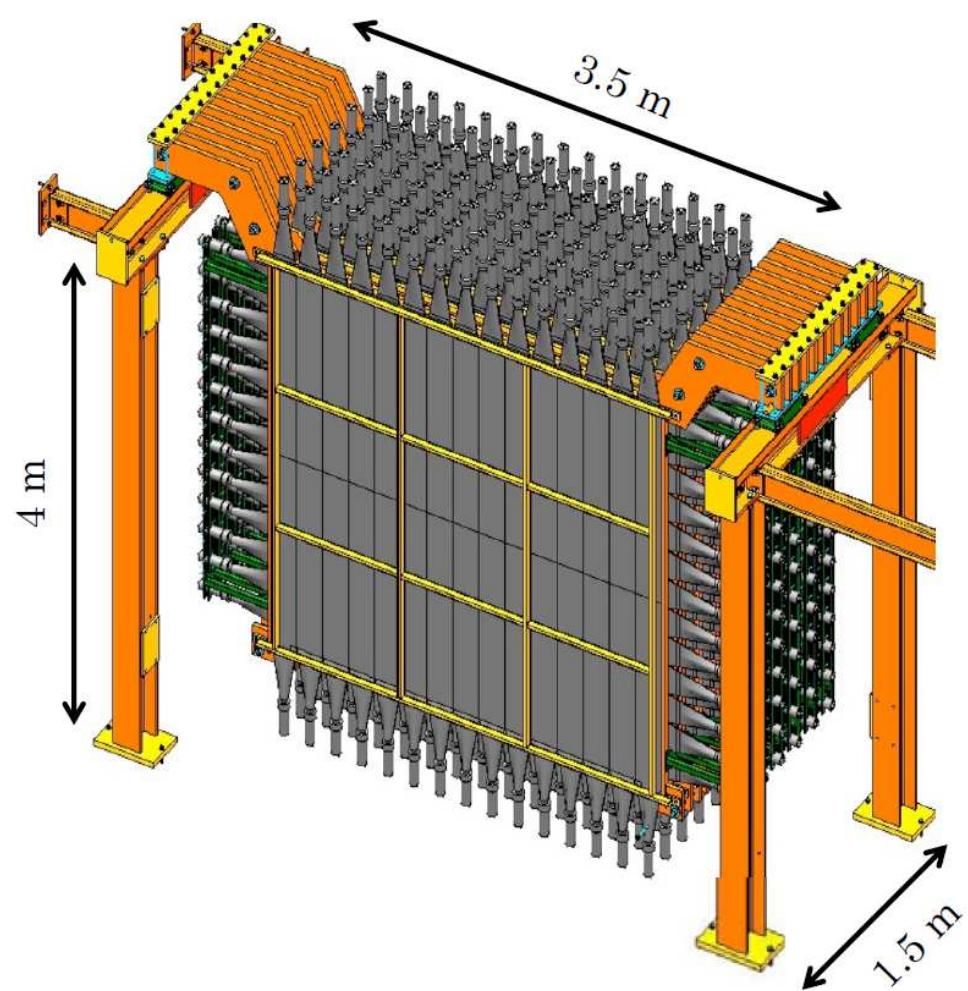

Figure 3.7: The MRD is installed downstream of the EC. It has 12 iron plates with thickness of $5 \mathrm{~cm}$ and 13 plastic scintillator planes with thickness of 6 mm. 
sionally on online monitor in the beam trigger, but most of the event display of the beam trigger have only pedestal (no hits). Figure 3.8 is a neutrino event candidate on online display. Vertical (left) and horizontal (right) readout of detectors are shown.
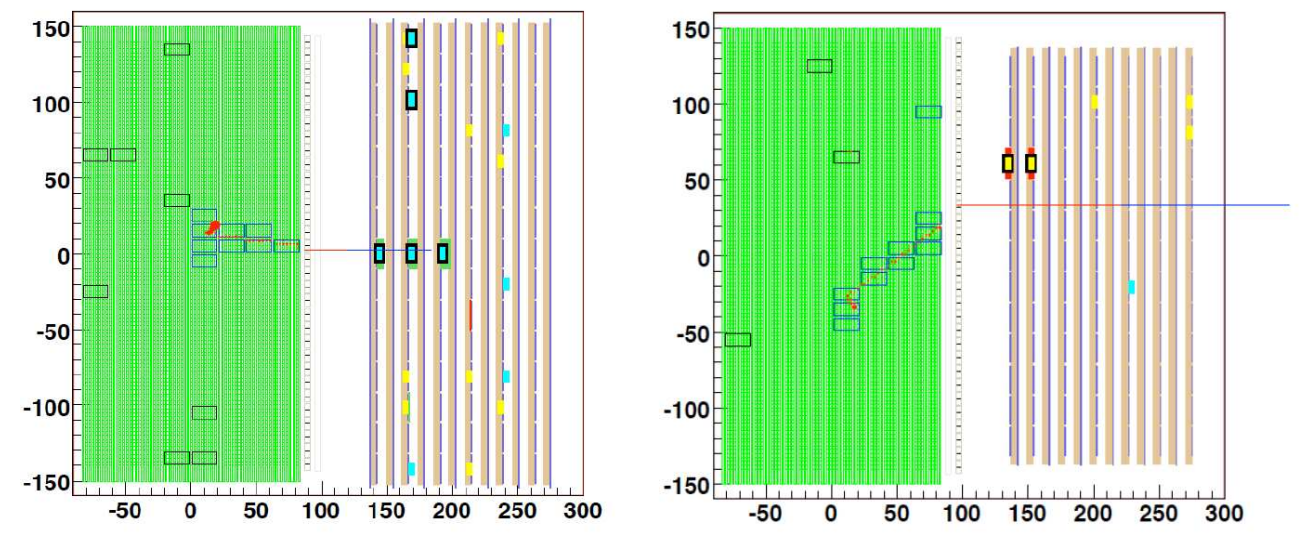

Figure 3.8: A neutrino event candidate on online display. Vertical (left) and horizontal (right) readout of detectors are shown.

\subsection{Neutrino Events in the SciBar Detector}

Figures 3.9 and 3.10 show charged current quasi elastic (CCQE) event candidates from actual data. The area of dots is propotional to the ADC channel (i.e. energy deposit). Boxes represent TDC hit information by their colors. The track penetrating the MRD in Figure 3.9 is identified as muon. One of the tracks in Figure 3.10 is identified as muon which decayed inside the SciBar using the multi-hit TDC information. Existence of muon is the sign of charged current events. Difference in $d E / d x$ between muons and protons can be seen. $d E / d x$ distributions of muons and protons are shown in Figure 3.11. Protons are separated from muons with $\sim 90 \%$ efficiency.

CC $1 \pi$ production can be categorized into three different groups: nuclear coherent interaction $\nu A \rightarrow \mu A \pi$, neutron scattering $\nu n \rightarrow \mu^{-} n \pi^{+}$and proton scattering $\nu p \rightarrow \mu^{-} p \pi^{+}$. As shown in Figure 3.12, scattering on neutrons or coherent interactions appears as two charged tracks with a common vertex in the SciBar, while scattering on proton appears as three charged tracks as in Figure 3.13, If one of the tracks is not long enough to be tracked in the proton interaction event, i.e. as is the case for the proton track in Figure 


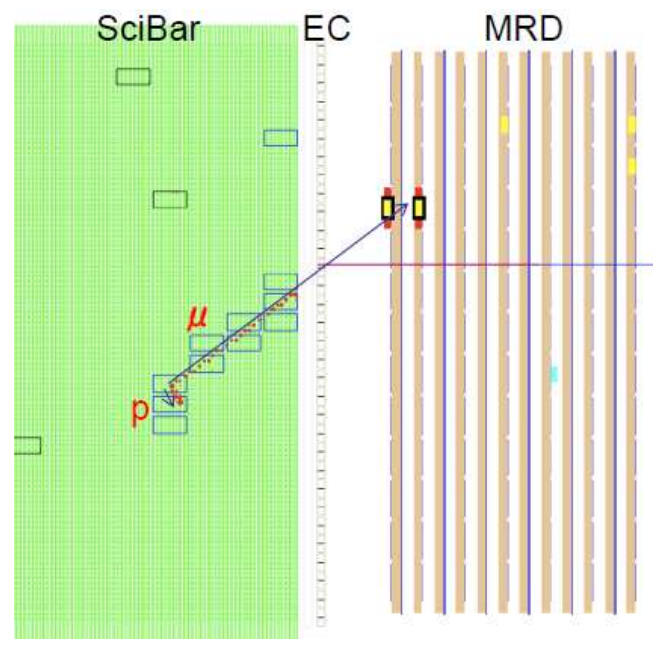

Figure 3.9: Event displays of CCQE candidate. The area of dots is proportional to ADC channels. Boxes represent TDC hit information. Tracks penetrating the MRD are identified as a muon.

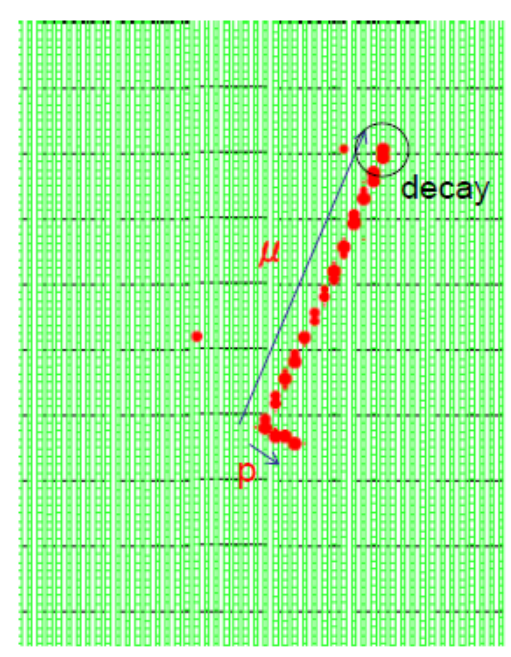

Figure 3.10: Event displays of CCQE candidate. The decay of the muon is tagged by multiple TDC hits.
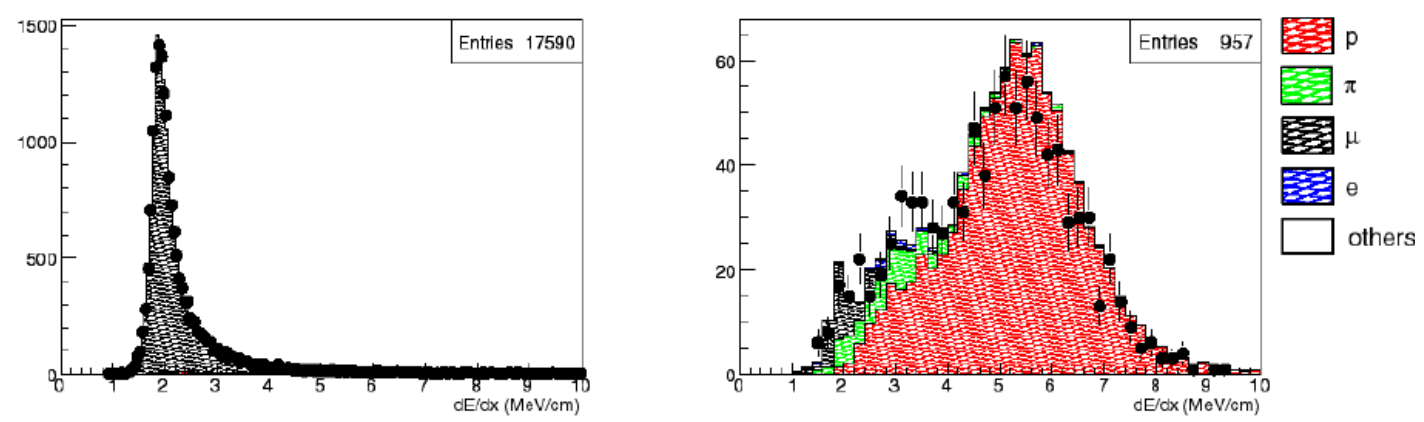

Figure 3.11: $d E / d x$ distributions of muons (left plot) and protons (right plot). Protons are separated from muons with $\sim 90 \%$ efficiency. 
3.13, the event can be distinguished from the neutron or coherent scattering events by looking at large energy deposit near the vertex.

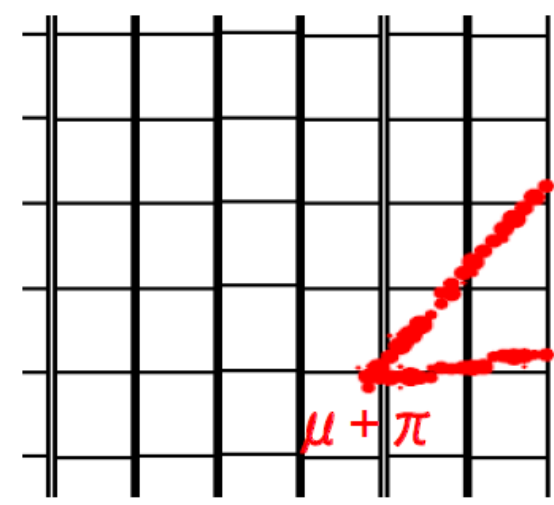

Figure 3.12: CC $1 \pi$ coherent or $\nu n \rightarrow \mu^{-} n \pi^{+}$candidate. Muon and pion are visible.

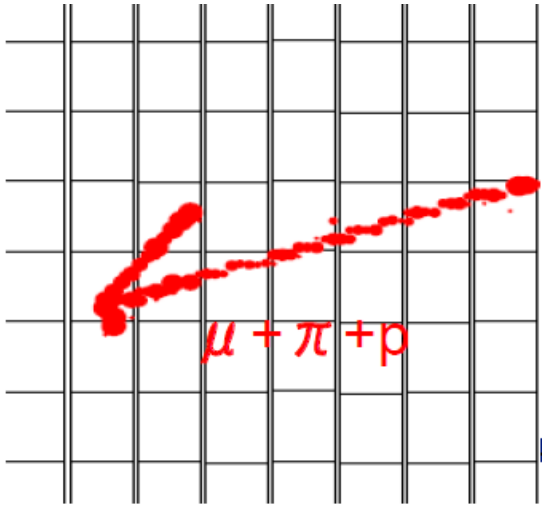

Figure 3.13: $\mathrm{CC} 1 \pi \nu p \rightarrow \mu^{-} p \pi^{+}$ candidate. Muon, pion and proton are visible.

There is no muon in the final state of neutral current events. Figure 3.14 shows a candidate of neutral current elastic scattering. The signature of this event is a single proton track. The proton is identified using $d E / d x$ information. Figure 3.15 shows a candidate of neutral current $\pi^{0}$ production event. The $2 \gamma^{\prime}$ 's from a $\pi^{0}$ decay produce electro-magnetic showers which can be detected in SciBar. These features of SciBar enable us to make precise measurements of neutrino-nucleus interactions in the region of neutrino energy below $1 \mathrm{GeV}$.

\subsection{Data Taking}

We started data taking in June 2007 with antineutrino mode, switched to neutrino mode in October 2007. We completed taking data of projected protons on target (POT) for neutrino mode in April 2008, then switched back to antineutrino mode. We completed taking data of antineutrino mode in August 2008. So, the total beam time was about 13 months. 


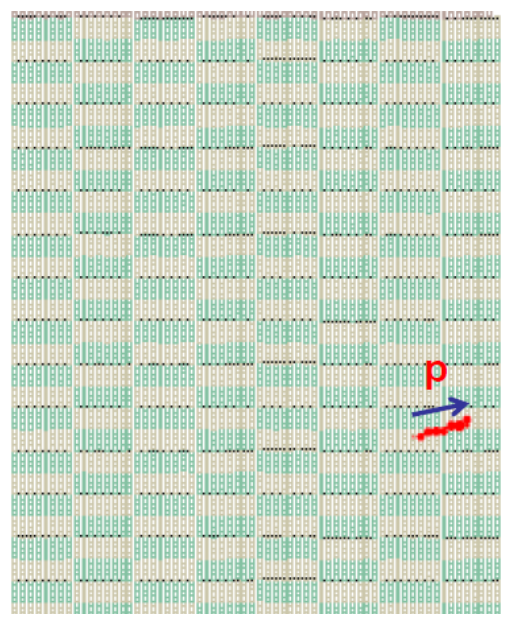

Figure 3.14: Candidate of neutral current elastic scattering. Signature of this event is single proton track. The proton is identified using $d E / d x$ information.

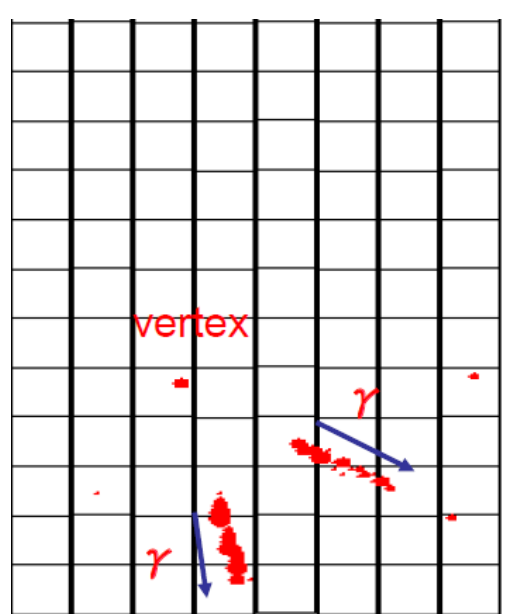

Figure 3.15: Candidate of neutral current $\pi^{0}$ production event. The $2 \gamma^{\prime}$ 's from a $\pi^{0}$ decay produce electro-magnetic showers, which can be detected in the SciBar.

Accumulated number of POT and number of POT which passed all data quality cuts are shown in Figure 3.16. $2.64 \times 10^{20}$ POT were delivered all together. Beam quality cuts are applied to all data. Beam intensity is required to be at least $0.1 \times 10^{12}$ protons per spill. The agreement between the readout of two troids need to be within $10 \%$. The absolute peak horn current is required to be greater than $170 \mathrm{kA}$. The targeting efficiency is required to be greater than $95 \%$. The efficiency of all the beam quality cuts is greater than $99 \%$. After applying all (beam and detector) data quality cuts, data of $2.52 \times 10^{20}$ POT are selected for physics analysis. As for neutrino data, $0.99 \times 10^{20}$ are selected. The rest is antineutrino data.

The number of CC event candidates is evaluated from tracks starting inside the SciBar every week during the data taking period. With the obtained number, one can monitor the SciBar status. Figure 3.17 shows number of $\mathrm{CC}$ event candidate normalized by POT. The flat shape for each mode and same values for the two antineutrino mode periods shows that the SciBar took data stably since we started data taking. Neutrino event rate is $\sim 4.5$ times larger than antineutrino event rate because of the difference between cross-sections of neutrino-nucleus and antineutrino-nucleus scattering, and production cross-sections of $\pi^{+}$and $\pi^{-}$that are parent particles of neutrino and antineutrino beam. 


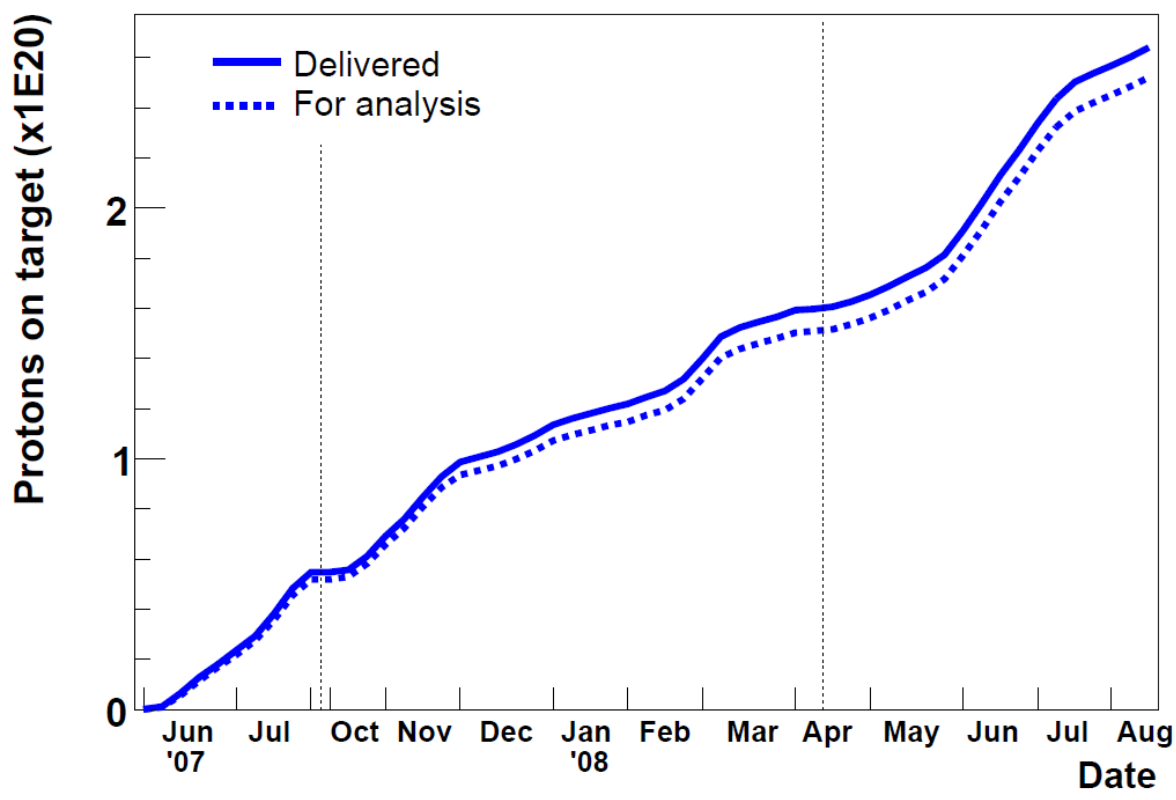

Figure 3.16: Accumulated number of POT (solid line) and number of POT which passed all the data quality cuts (dotted line). 


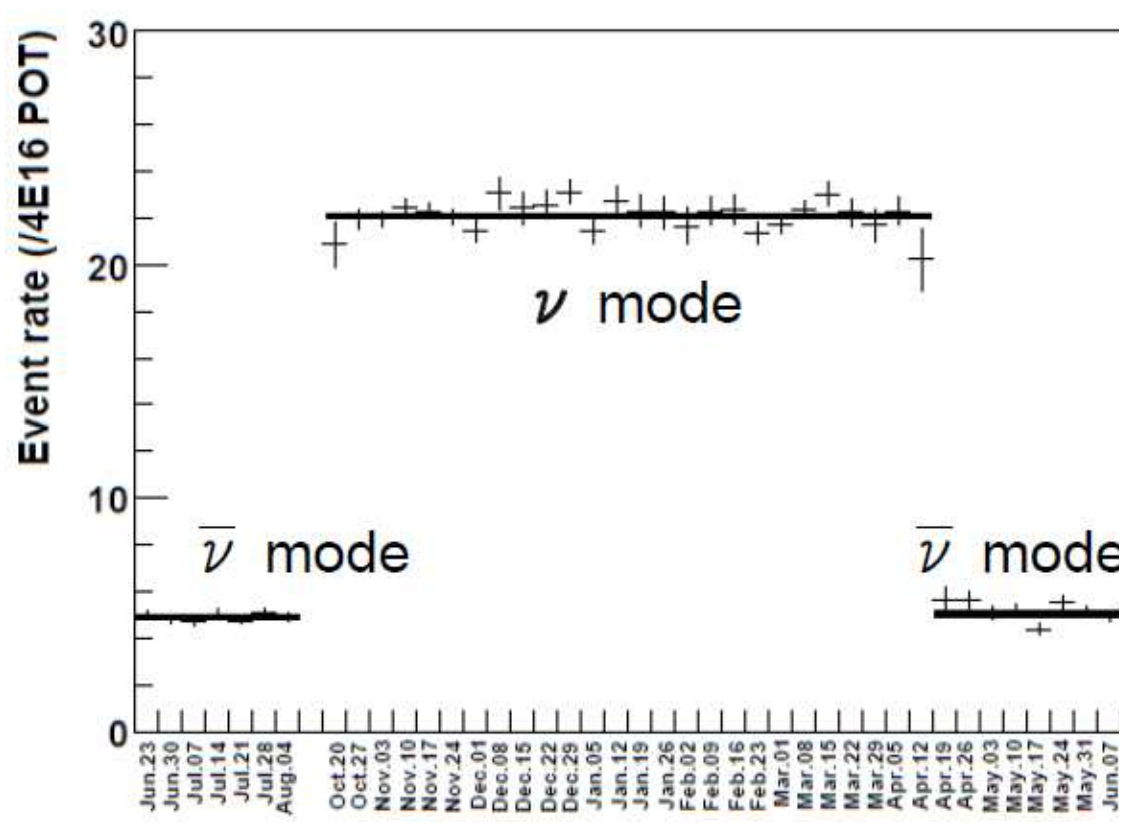

Figure 3.17: Number of CC event candidates normalized by POT. The flat shape for each mode and the same values for the two antineutrino mode periods shows that the SciBar took data stably throughout the beam time. 


\subsection{Beam Simulation}

Neutrino flux at the SciBooNE is predicted using GEANT4-based [19] beam Monte Carlo simulation. SciBooNE uses the same simulation code developed by the MiniBooNE Collaboration [20] at FNAL.

The primary protons are generated according to the beamline optics upstream of the target. The interactions of primary protons with the beryllium target are simulated according to state-of-the-art hadron interaction data. The geometry and materials of all objects in the target hall and decay region are modeled in the simulation code.

Predictions of the secondary particles production on beryllium target are important for neutrino flux predictions. Experimental data of hadron interaction from HARP [21] and BNL E910 [22] are used. Production of secondary protons, neutrons, charged pions, and charged and neutral kaons is taken into account. Elastic and quasi-elastic scatterings of protons in the target are also simulated. Particles from the primary proton-beryllium target interactions are simulated by the GEANT4 framework taking into account all the physics process. Hadronic re-interactions of pions and nucleons with beryllium target and aluminum materials are particularly important. They are described by recently updated models. A Sanford-Wang parameterization [23] describes the double-differential inclusive production cross-section for pions and neutral kaons. The Sanford-Wang parameterization of the inclusive production cross section is given by

$$
\frac{d^{2} \sigma}{d p d \Omega}=c_{1}\left(1-\frac{p}{p_{B}-c_{9}}\right) \exp \left[-c_{3} \frac{p^{c_{4}}}{p_{B}^{c_{5}}}-c_{6} \theta\left(p-c_{7} p_{B} \cos ^{c_{8}} \theta\right)\right]
$$

where $p$ and $\theta$ are the meson momentum and angle, $p_{B}$ is the beam momentum, and the constants $c_{1}, c_{2}, \ldots, c_{9}$ are parameters determined from fits to meson production data from HARP and BNL E910. The Feynman scaling parameterization describes the $\mathrm{K}^{+}$production cross section [24]. The Feynman scaling parameterization is given by

$$
\begin{aligned}
\frac{d^{2} \sigma}{d p d \Omega}= & \frac{P_{K}^{2}}{E_{K}} c_{1}\left(1-\left|x_{F}\right|\right)^{c_{3}} \exp \left[-c_{3}\left|x_{F}\right|^{c_{4}}\right. \\
& \left.-c_{7}\left|p_{t} * x_{F}\right|^{c_{6}}-c_{2} p_{t}-c_{5} p_{t}^{2}\right]
\end{aligned}
$$

where $x_{F}=p_{\|}^{C M} / p_{\|}^{C M, \max }$ is the Feynman scaling parameter ( $p_{\|}$is longitudinal momentum), $p_{t}$ is the transverse momentum of the $\mathrm{K}^{+}$and the constants $c_{1}, c_{2}, \ldots, c_{7}$ are parameters determined from fits to experimental data [25] 26] 27] 28] [29] [30] 31] 32] 33]. The other hadronic processes and all electromagnetic processes are described by default GEANT4 physics lists. 
To simulate the neutrinos from the secondary particles (mesons), the output of the GEANT4 codes is used by the second MC code as input. The second MC generates the neutrino kinematics distributions from meson and muon decays and obtains the final neutrino fluxes extrapolated to the SciBooNE detector. Current best knowledge of neutrino-producing meson and muon decay branching fractions, and decay form factors in three-body semi-leptonic decays are used. Polarization effects in muon decays are also accounted for.

All neutrinos that enter the detectors are considered for SciBooNE flux predictions. Weights for each neutrino event is calculated using information of neutrino flavor, energy, parent type and kinematics. Information from the interaction and detector simulation such as neutrino interaction probability, detailed detector geometry and specifications are also used.

Neutrino flux prediction at the SciBooNE detector as a function of neutrino energy $E_{\nu}$ is shown in figure 3.18. A total neutrino flux per proton on target of $2.2 \times 10^{-8} \mathrm{~cm}^{-2}$ is expected at the SciBooNE detector location in neutrino mode. Mean neutrino energy is predicted to be $0.7 \mathrm{GeV}$. 93\% of total flux is muon neutrinos. Fraction of muon antineutrinos is $6.4 \%$ and electron neutrinos and antineutrinos is $0.6 \%$. 


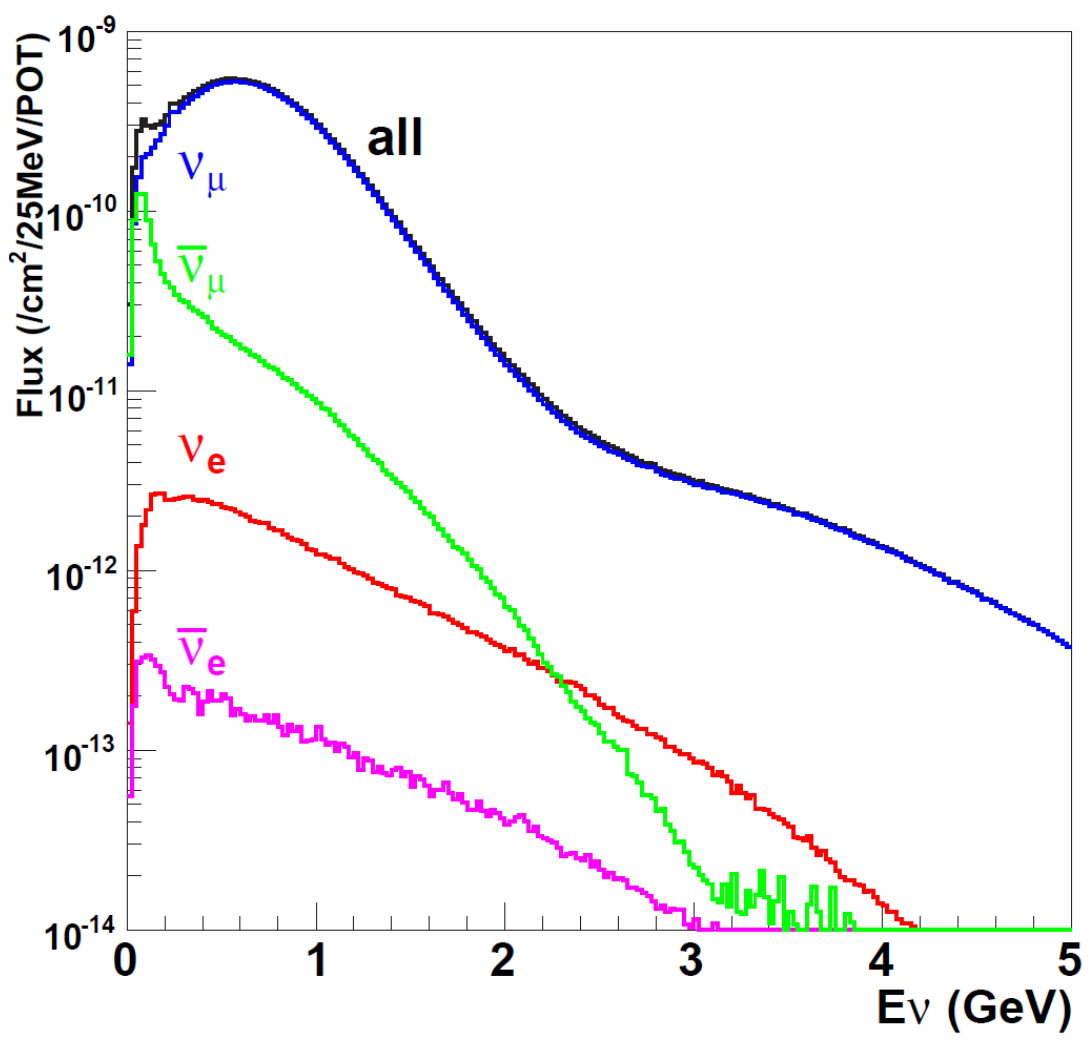

Figure 3.18: Neutrino flux prediction at the SciBooNE detector as a function of neutrino energy $E_{\nu}$. 


\subsection{Neutrino Interaction Simulation}

NEUT [34] 35] is a program for neutrino interaction simulation. Elastic scattering, single meson production, single gamma production, coherent pion production and deep inelastic scattering in both neutral and charged currents are simulated by NEUT. Nuclear effects are also considered. NEUT handles protons, oxygen, carbon and iron as nuclear targets. The energy range for the simulation is from $100 \mathrm{MeV}$ to $100 \mathrm{TeV}$. Output from the beam simulation is used as input for the NEUT.

\subsubsection{Elastic Scattering}

Elastic scattering is implemented in NEUT using the model of LlewellynSmith [36]. Fermi gas model of Smith and Moniz [37] is used for scattering off nucleons in the nucleus. The Fermi motion of nucleons along with the Pauli exclusion principle is taken into account. The momentum distribution of the target nucleon is assumed to be flat up to a fixed Fermi surface momentum of $217 \mathrm{MeV} / \mathrm{c}$ for carbon and $250 \mathrm{MeV} / \mathrm{c}$ for iron. The same Fermi momentum distribution is also used for all other nuclear interactions. The nuclear potential is set to be $27 \mathrm{MeV}$ for carbon and $32 \mathrm{MeV}$ for iron. Both vector and axial-vector form factor are assumed to be dipole. The vector mass in elastic scattering is set to be $0.84 \mathrm{GeV} / c^{2}$. The axial vector mass is set to be $1.11 \mathrm{GeV} / c^{2}$. The $Q^{2}$ dependence of the differential cross-section for NC elastic scattering in NEUT is shown in Figure 3.19, Black line and red line shows differential cross-sections for neutrino-proton scattering and neutrino-neutron scattering respectively.

\subsubsection{Resonant Single Meson Production}

Resonant production of pions, kaons and etas via baryon resonances are described by the model of Rein and Sehgal [38. The model assumes an intermediate baryon resonance, $N^{*}$, in the reaction of $\nu N \rightarrow l N^{*}, N^{*} \rightarrow$ $N^{\prime} m$. The differential cross-section for single meson production depends on the amplitude for the production of a given resonance. The probability of the baryon resonances with mass less than $2 \mathrm{GeV} / c^{2}$ is included. Those baryon resonances with mass greater than $2 \mathrm{GeV} / c^{2}$ are simulated as deep inelastic scattering. Lepton mass effects from the non-conservation of lepton current and the pion-pole term in the hadronic axial vector current are included in the simulation [39] 40]. To determine the angular distribution of a pion in the final state, Rein's method [41] is used for the $P_{33}(1232)$ resonance. For other resonances, the directional distribution of the generated pion is set to 


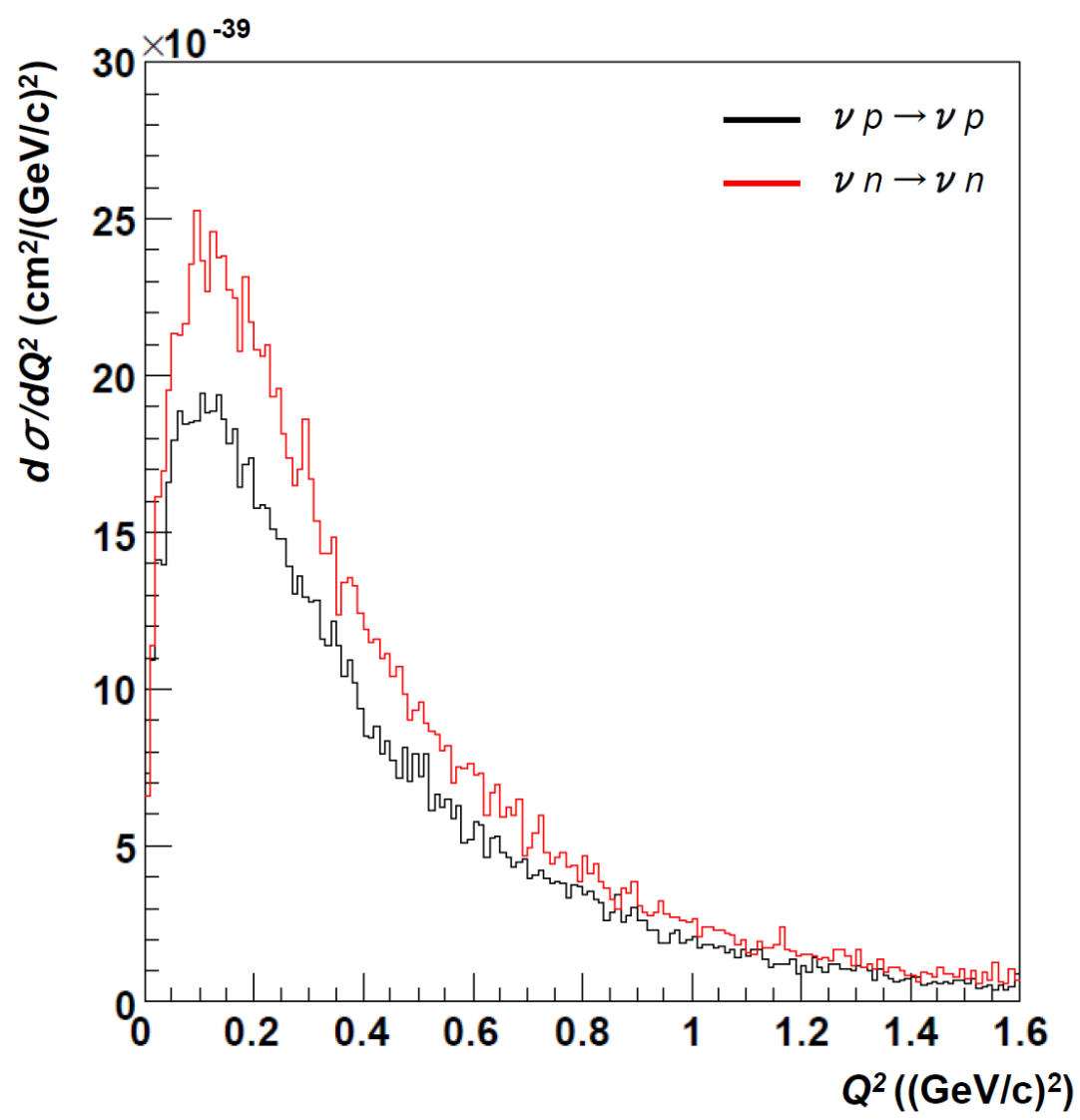

Figure 3.19: The $Q^{2}$ dependence of the differential cross-section for NC elastic scattering adopted in NEUT. Black line and red line shows differential cross-sections for neutrino-proton scattering and neutrino-neutron scattering respectively. 
be isotropic in the resonance rest frame. The angular distribution of $\pi^{+}$has been measured for $\nu_{\mu} p \rightarrow \mu^{-} p \pi^{+}$[42] and the results agree well with NEUT's prediction.

Pauli blocking is accounted for in the decay of the baryon resonance by requiring the momentum of the nucleon to be larger than the Fermi surface momentum. Pion-less $\Delta$ decay is also taken into account, where $20 \%$ of the events do not have a pion [43].

\subsubsection{Coherent Pion Production}

Coherent pion production is a neutrino interaction with a nucleus which remains intact, creating one pion with the same charge as the incoming weak current. Rein and sehgal model [44] with lepton mass correction [46] and Kartavtsev et al. model [47] are used to simulate the coherent pion production.

\subsubsection{Nuclear Effects in Final State}

NEUT simulates the interactions of the generated mesons and nucleons in nucleus. Nucleons which have obtained recoil energy in nucleus interacts with other nucleons bound in the same nucleus. The nucleon-nucleon elastic scattering cross section is based on the measurements by Bertini et al. [48]. The pion production from the decay of produced $\Delta$ is also taken into account according to the isobar production model [49. Probability of nucleon-nucleon interaction in ${ }^{16} \mathrm{O}$ after the neutrino interaction is shown in figure 3.20 . No interaction, elastic scattering, single $\pi$ production, and two $\pi$ production are plotted.

The production of nucleons with nucleon momentum below $225 \mathrm{MeV} / \mathrm{c}$ is suppressed by the Pauli blocking effect. Momentum above $300 \mathrm{MeV} / \mathrm{c}$, about a half of the nucleons interact mainly by elastic scattering. Mean deflection angle is shown in figure3.21. It has a peak around 45 degrees.

The concept of formation zone is also considered for all hadrons generated in nucleus. This is a distance or time from the neutrino interaction point to the hadron production point. The intermediate states are assumed to be non-bound quark states. The formation length of each hadron is expressed as

$$
L=p / \mu^{2}
$$

where $p$ is the momentum of the hadron and $\mu^{2}=0.08 \mathrm{GeV}^{2}$ is a fitted constant from the SKAT experiment [50]. We can see the effect of nucleon-nucleon 


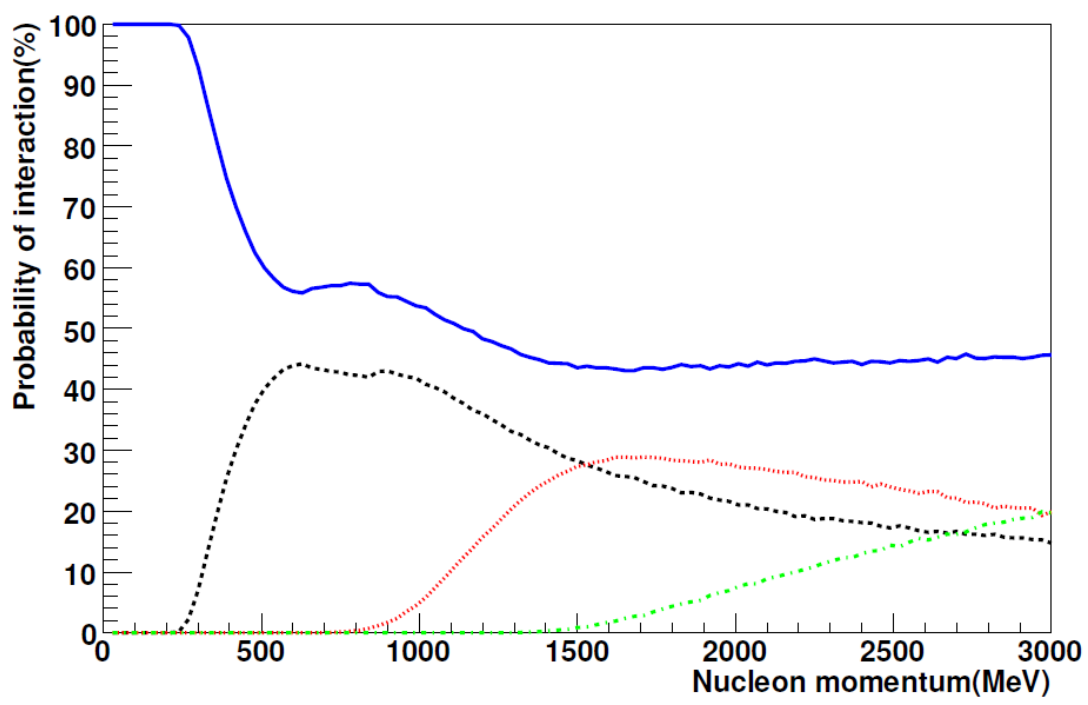

Figure 3.20: Probability of nucleon-nucleon interaction in ${ }^{16} \mathrm{O}$ after the neutrino interaction. No interaction (solid line), elastic scattering (dashed line), single $\pi$ production (dot-dot line), and two $\pi$ production (dash-dod line) are plotted. 


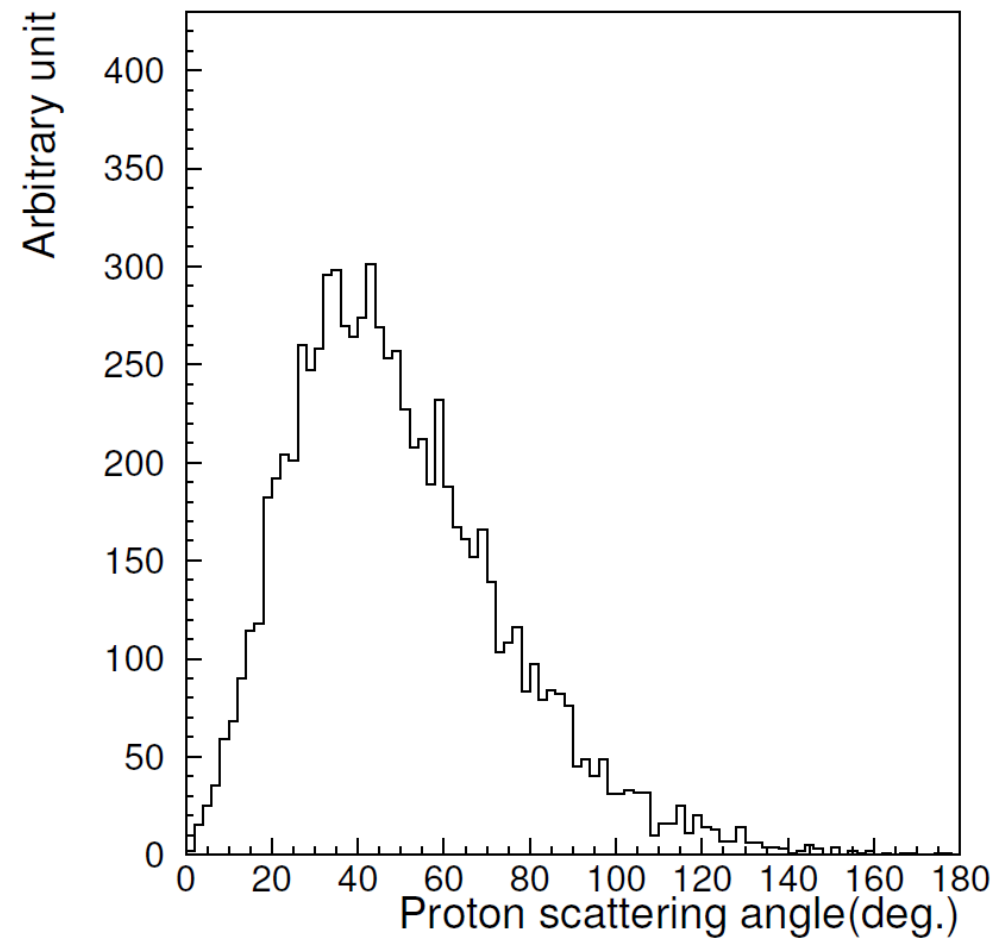

Figure 3.21: Mean deflection angle. It has a peak around 45 degrees. 
interactions in the nucleus in the significant distortion of the geometry of particles observed in detector.

\subsection{Detector Simulation}

The GEANT4 framework is used for the detector simulation. Output from neutrino interaction simulation is used as input for the detector simulation. Geometries of the detectors, the detector frame and experimental hall are based on survey measurements which were carried out during detector construction period.

\section{Particle Simulation in the Detectors}

For the hadronic interactions in the detectors, the Bertini cascade model in GEANT4 [51] is used.

The details of the nuclear model are discribed in reference [52] [53]. The motion of the bound nucleons, the Pauli exclusion principle, the diffuseness of the nuclear edge, and a local potential for nucleons and pions are included in the model. Data from several free-particle experiments are used for the crosssections for nucleon-nucleon and nucleon-pion reactions in the Bertini cascade model [54 [55] [56] 57] [58] [59] 60] 61] 62] 63]. Figure 3.22 shows proton-proton total and elastic cross sections. Figure 3.23 shows the same plot for protonneutron. Where these data were lacking, they were estimated by use of isospin symmetry or phase-shift analysis.

\section{Detector Response}

For the SciBar detector simulation, information of particles generated by NEUT is used as input to the simulation. The energy loss of a charged particle in a single strip of SciBar is simulated by GEANT. This energy scale is tuned using cosmic ray data. Scintillator quenching is simulated using Birk's law [64] with a value of Birk's constant of $0.0208 \mathrm{~cm} / \mathrm{MeV}$ measured at K2K [65]. The energy deposited by a charged particle is converted to photoelectrons using conversion factors. Conversion factors are measured for each channel of SciBar with cosmic muons. The measured light attenuation length of each fiber is used in the simulation. Average light attenuation length is approximately $350 \mathrm{~cm}$. Cross-talk between nearby MAPMT channels is measured in laboratory and used in the simulation. The number of photoelectrons is smeared by Poisson statistics. The single photoelectron resolution of the MAPMT is simulated. The number of photoelectrons is 


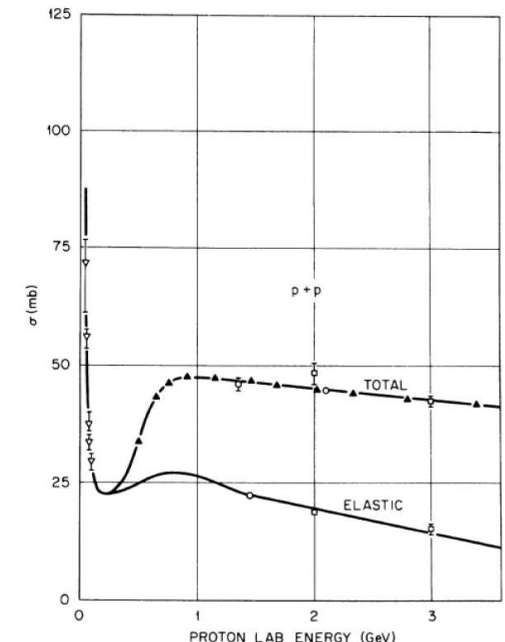

A, D. V. Bugg et al. (see Ref. 15); $\nabla$, U. E. Kruse et al. quoted by F. F. Chen, C. P. Leavitt, and A. M. Shapiro Phys. Rev. $\underline{103}, 211(1956)$; $\square$, T. Ferbel et al., in Proceedings of the 1962 International Conference on HighEnergy Physics at CERN, edited by J. Prentki (CERN, Geneva, 1962), p. 76 ; $O$, S. P. Kruchinin et al., Yader Fiz. 1, 317 (1965) [transl.: Soviet J. Nucl. Phys. 1, 225

Figure 3.22: Proton-proton total and elastic cross sections.

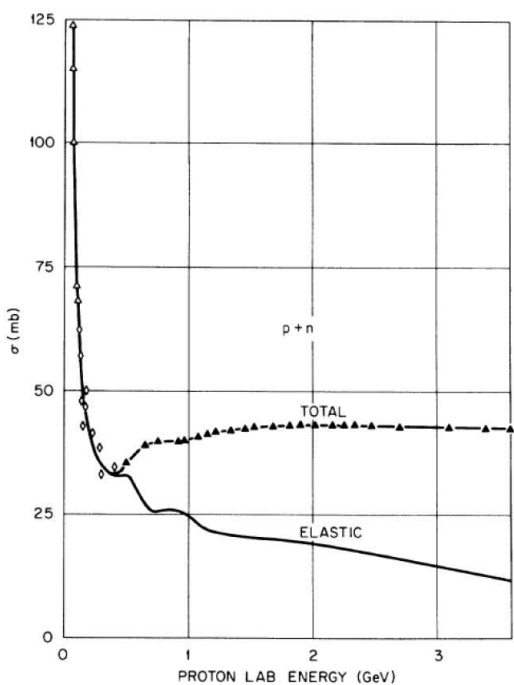

A, D. V. Bugg etal. (see Ref. 15); $\diamond$, J. H. Atkinson et al., Phys. Rev. 123, 1850 (1961); $\triangle$, P. H. Bowan et al., Nucl. Phys. 22,640 (1961).

Figure 3.23: Proton-neutron total and elastic cross sections. 
converted to ADC channels, and then electronics noise and threshold effects of the TA are simulated.

TDC hit simulation includes light propagation delays in the WLS fibers. A logical OR of 32 MAPMT channels is made for each TDC channel, and the time of each hit is converted to TDC channels. Multiple TDC hits in each channel are simulated.

In the EC detector simulation, true energy deposition in scintillating fibers in the detector is converted to the number of photoelectrons using the conversion factor. The conversion factor is measured for each channel with cosmic muons. The attenuation of light in the fiber is simulated using the measured attenuation light value. The number of photoelectrons is smeared by Poisson statistics and by the PMT resolution, and then converted to $\mathrm{ADC}$ counts. The time-dependent $\mathrm{ADC}$ gain due to the overshoot of the PMT signal is simulated based on a measurement with cosmic muons. Electronics noise is also simulated.

For the MRD detector simulation, true energy deposition in each scintillator is converted to ADC channels using the conversion factor measured with cosmic muons. The attenuation of light in the scintillator and electronics noise are simulated. Gaps between scintillator counters in each plane are included in the simulation. The time of energy deposition is digitized and converted into TDC channels.

\subsection{Dirt Simulation}

Recoiled particles recoiled by neutrinos inside dirt or wall of experimental hall around the detector sometimes enter the detector and make hits or interact with particles inside the detector. These events are called 'dirt events'. Dirt events mimic neutrino interactions in SciBar and can become backgrounds. For NC elastic analysis, the major background is protons recoiled by neutrons from the dirt (dirt neutron). Since both the signals and dirt neutron events are single proton track, it is difficult to distinguish the signals from dirt neutron events. To estimate backgrounds from dirt events, they are simulated using the beam simulation, NEUT and the detector simulation. The beam simulation is extended to cover a volume of $10 \times 10 \times 10 \mathrm{~m}^{3}$, taking the detector origin as a center (figure 3.24). Gray areas indicate the dirt volume. For NEUT, carbon is used for the material of the dirt with density 2.15 $\mathrm{g} / \mathrm{cm}^{3}$. For detector simulation, default composition of concrete which is available in GEANT4 is used for the material of the dirt. Density is 2.15 $\mathrm{g} / \mathrm{cm}^{3}$ based on a survey measurement. In this simulation, same material and density are used for dirt and walls of the experimental hall. 

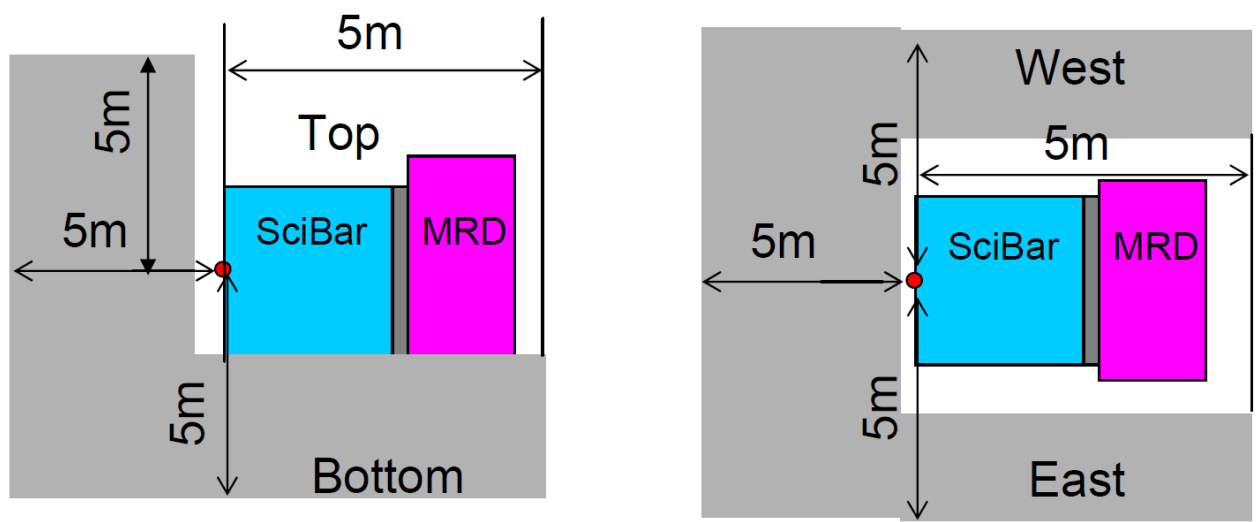

Figure 3.24: Geometry of dirt for the dirt simulation. Gray areas indicate dirt volume. The geometry of $10 \times 10 \times 10 \mathrm{~m}^{3}$, taking detector origin (red point) as a center were considered. 

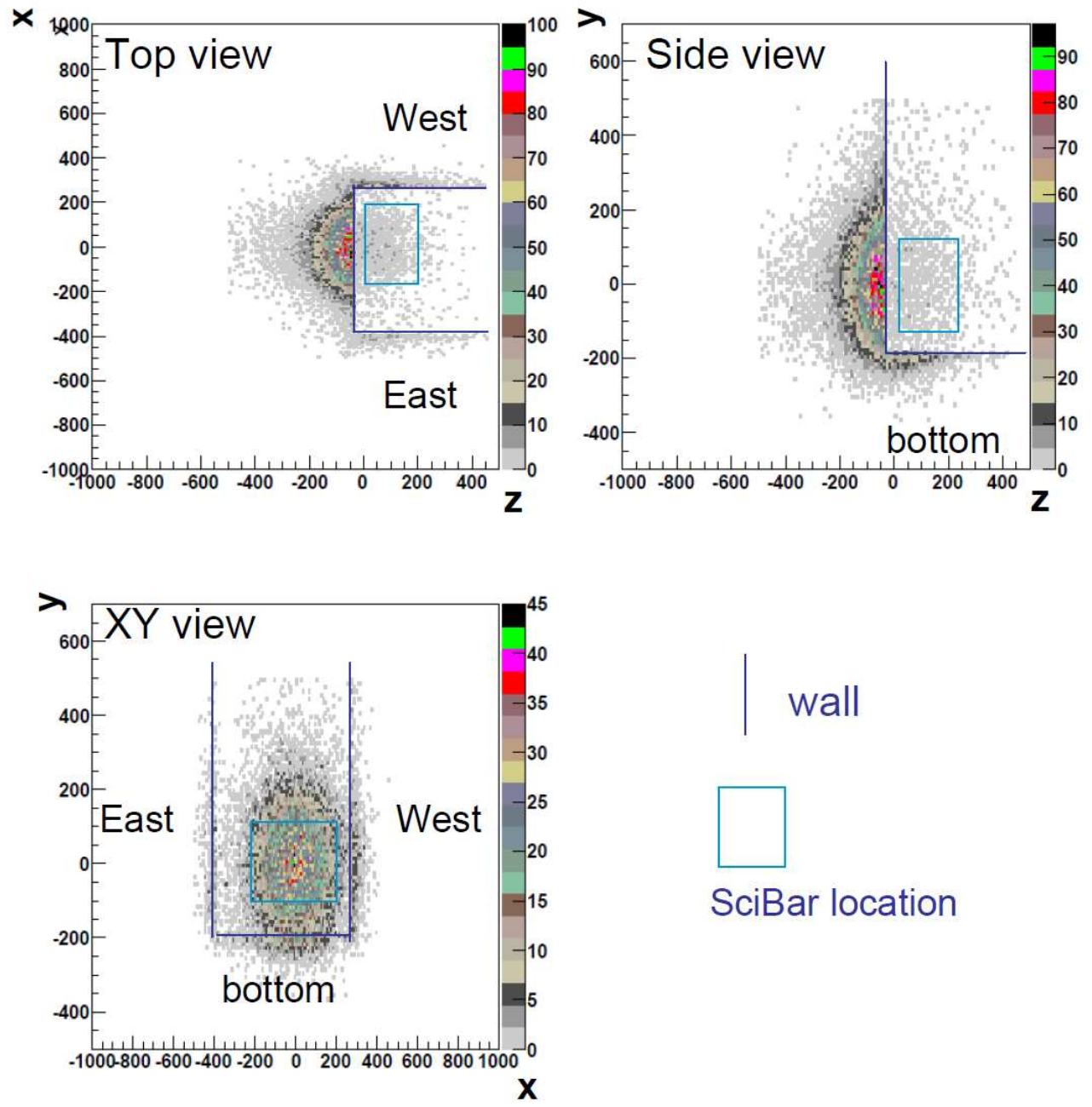

Figure 3.25: Vertex distributions of dirt events from simulation. Only events which made one track contained in SciBar were selected. Geometry is large enough for NC elastic analysis. 


\section{Chapter 4}

\section{Analysis of Neutral Current Elastic Scattering}

Figure 4.1 shows a candidate of neutral current elastic scattering from SciBooNE data. Signature of this event is single proton track. The proton is identified using the stopping range and $d E / d x$ informaiton. Analysis for evaluating the cross section for neutrino-nucleon neutral current elastic scattering is performed.

Flow chart of this analysis is shown in Figure 4.2.

\subsection{Track Reconstruction}

Tracks in SciBar are reconstructed as two-dimentional (2D) tracks in each view of SciBar using a cellular automaton algorithm [66. Three dimentional (3D) tracks are reconstructed by matching the timing of $2 \mathrm{D}$ tracks and $z$ position of track edges. The conditions for matching requires the timing difference between two $2 \mathrm{D}$ tracks to be less than $50 \mathrm{~ns}$ and the $z$-edge difference to be less than $6.6 \mathrm{~cm}$ for both upstream and downstream edges. The hit threshold for tracking is set to two photoelectrons. Reconstructed tracks are required to penetrate at least three layers. Due to this requirement, the minimum length of a reconstructed track is $8 \mathrm{~cm}$.

\subsection{Monte Carlo Normalization}

All MC events used in this analysis is normalized with a data over MC ratio of number of CC events $N^{c c} / N_{M C}^{c c}$. CC events are identified by matching a track in SciBar and a track or hits in the MRD. The track which satisfied this condition is called SciBar-MRD matched track. The most energetic 


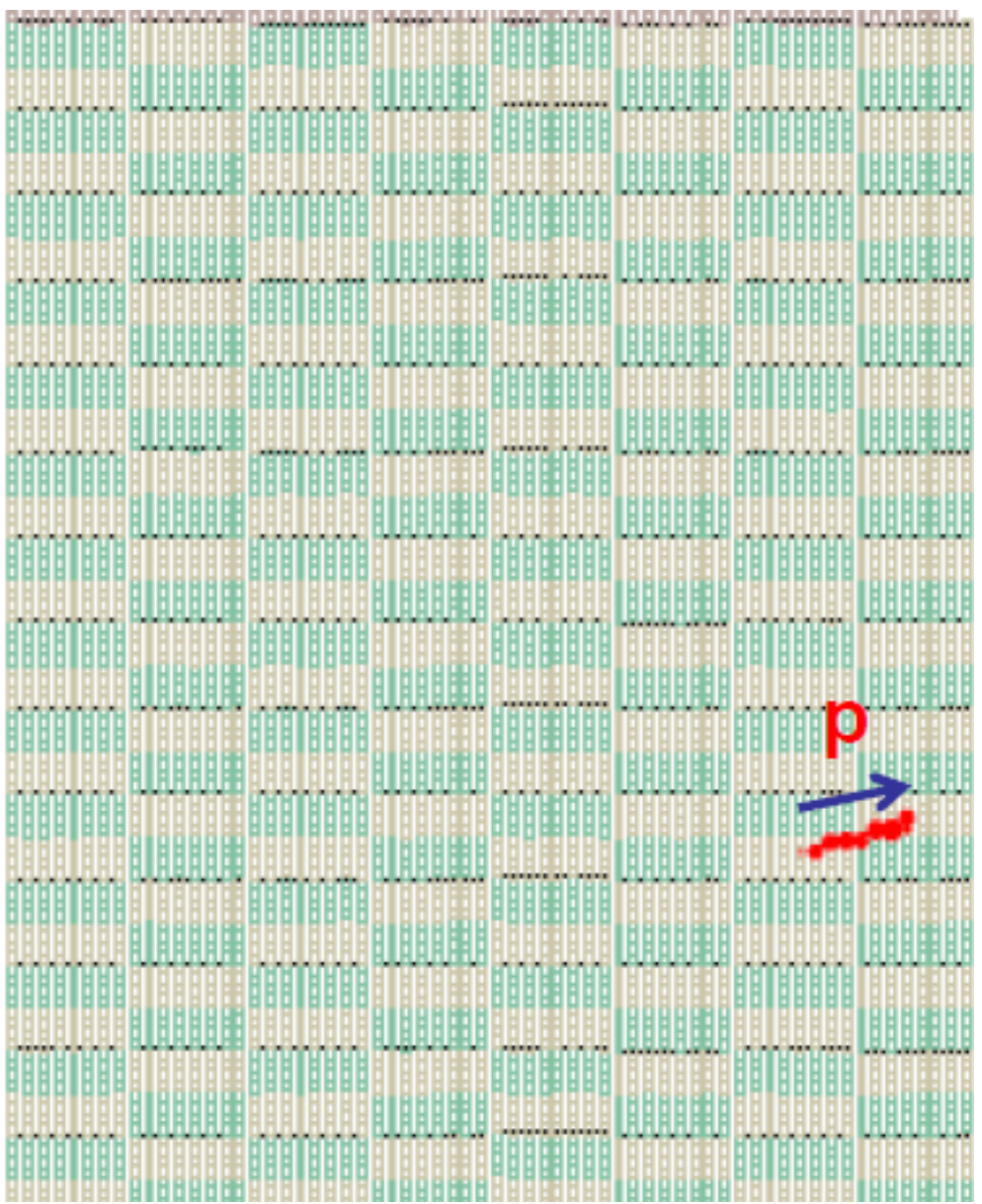

Figure 4.1: A candidate of neutral current elastic scattering from SciBooNE data. Signature of this event is single proton track. The proton is identified using stopping range and $d E / d x$ information. 


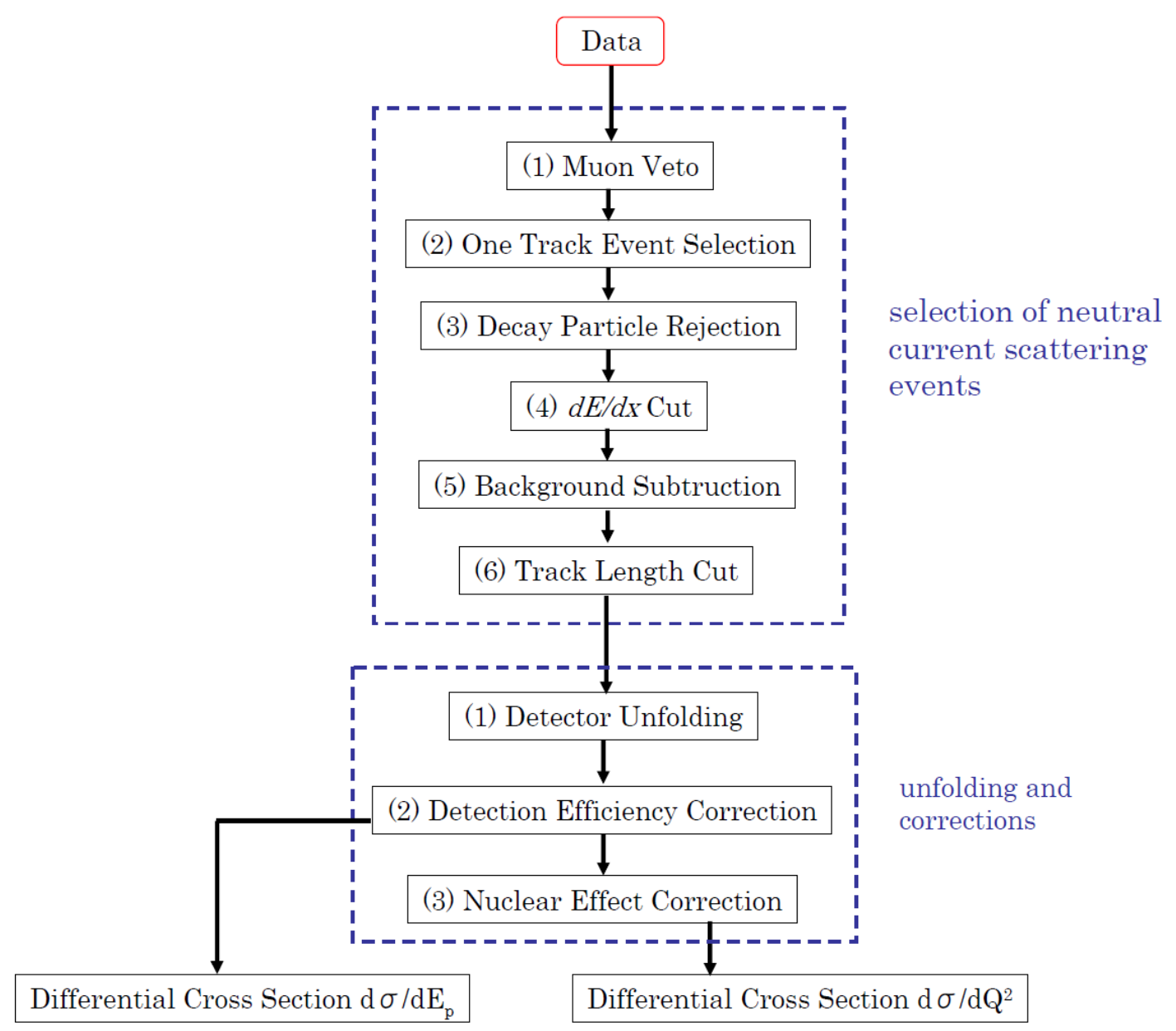

Figure 4.2: Flow chart of this analysis. 
SciBar-MRD matched track is considered as a muon candidate. A MRD track is required to have the upstream track edge on the first two layers of the MRD. The transverse distance between the two tracks at the first layer of the MRD is required to be less than $30 \mathrm{~cm}$. The requirement on the difference between track angles with respect to the beam direction is given by $\left|\theta_{M R D}-\theta_{\text {SciBar }}\right|<\theta_{M A X}$ where $\theta_{M A X}$ is a function of the length of the MRD track. $\theta_{M A X}$ varies between 0.4 to 1.1 radians. At least two layers with hits in each view are required for track reconstruction in the MRD. If no MRD track is found, the SciBar track is extrapolated to the MRD and search for nearby contiguous hits in the MRD identifying a short muon track. For matching MRD hits to a SciBar track, the MRD hit is required to be within a cone with an aperture of \pm 0.5 radian and a transverse offset within $10 \mathrm{~cm}$ of the extrapolated SciBar track at the upstream edge of the MRD. The timing difference between the SciBar track and the track or hits in the MRD is required to be within $100 \mathrm{~ns}$. Lower limit of the momentum of muon with matched track is $350 \mathrm{MeV} / c$ due to these matching criteria.

\subsection{Event Selection}

Event selections using geometrical information and particle identification are performed to pick up NC elastic events in fiducial volume (FV). FV is defined from 3rd layer to 62 th layer, $\pm 130 \mathrm{~cm}$ from detector center along the axis perpendicular to the beam direction.

\subsubsection{Geometrical Selection}

\section{Veto}

Outside the FV of the SciBar is used for veto to avoid charged particles entering the SciBar from outside and escaping from inside the SciBar. Veto uses a clustering method. Definition of cluster is more than two hits with photoelectrons larger than 10 in a distance less than two cells. If there is any cluster in veto region and time difference between the track inside the FV and the cluster is less than $100 \mathrm{~ns}$, event is rejected. This method automatically selects tracks contained in FV. Tracks need to be contained so that kinetic energy of recoil proton is reconstructed correctly.

$\mathrm{EC}$ is also used for veto. The purpose of the $\mathrm{EC}$ veto is to reject $\mathrm{NC}$ $\pi^{0}$ events in which $2 \gamma$ from $\pi^{0}$ decay escape from SciBar without making electromagnetic shower. If there is a coincidence of a pair of PMTs of at least one EC module, event is rejected. It reduces $11.9 \%$ of backgrounds from $\mathrm{NC}$ pion production. 


\section{One Track Event Selection}

For NC elastic sample, only events with one track are selected. 2D track in both view are required to be single track. In case tracks do not penetrate three layers because of large angle or short length, they are not reconstructed. To detect such tracks, two additional methods are used. For large angle tracks, more than five continuous hits with 5 photoelectrons in the same layer are considered as one track. Time difference between reconstructed track and all hits are required to be less than 100 ns. Searching area of large angle track is from two layers upstream to two layers downstream of the vertex. For short tracks and other tracks that are not reconstructed, total energy deposit from track-unrelated hits is used. Total energy deposit is calculated for each view. Hits with more than 10 photoelectrons are used for energy deposit calculation to pick up minimum ionizing particles, considering light attenuation by WLS fibers. Figure 4.3.1 shows total energy deposit of track-unrelated hits on top and side of SciBar. The threshold to select one track events is set to $30 \mathrm{MeV}$ for both top and side view.
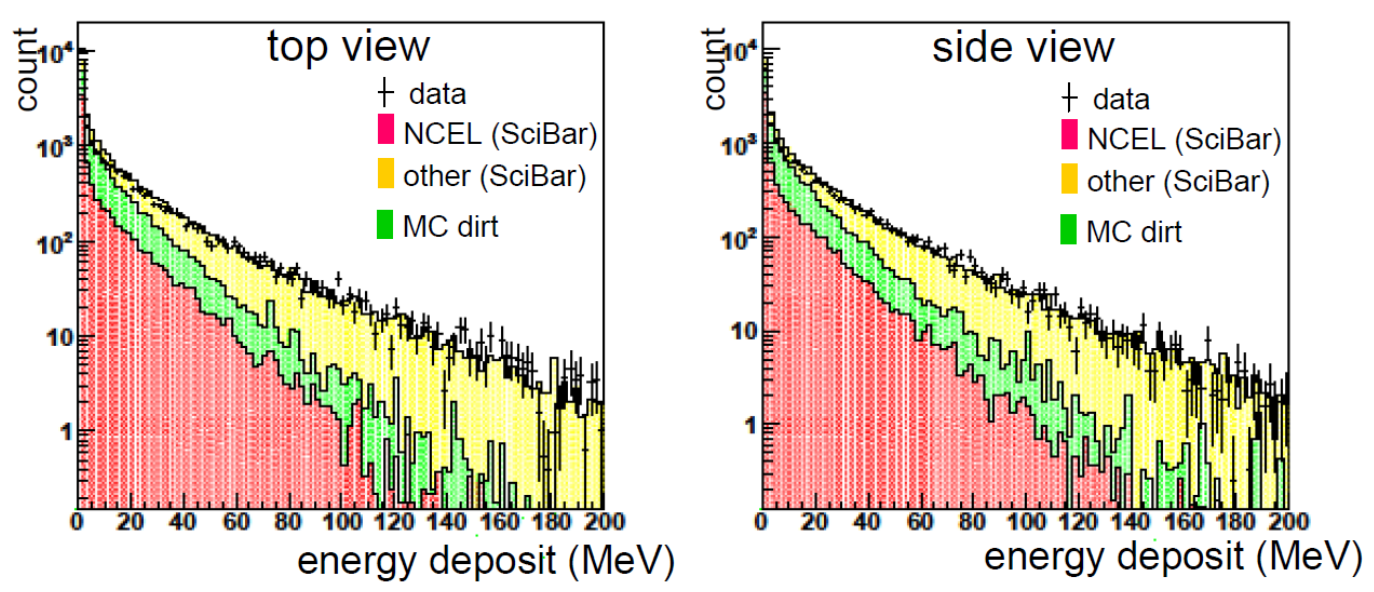

Figure 4.3: Total energy deposit of track-unrelated hits on top and side of SciBar. Data are compared to MC simulation. The threshold to select one track events is set to $30 \mathrm{MeV}$ for both top and side view. In these plots, dots with error bars is data and others are $\mathrm{MC}$ simulation. Red is $\mathrm{NC}$ elastic events, yellow is other interaction, and green is dirt events. 


\subsubsection{Particle Identification}

To identify protons, two types of process were performed: decay particle rejection and $d E / d x$ cut. Decay particle rejection uses multi-hit TDC information. $d E / d x$ cut uses energy deposit along the track and track length of reconstructed tracks.

\section{Decay Particle Rejection}

Muons and charged pions stopped inside SciBar FV is identified using multiple TDC hit. If a muon decays inside SciBar, there are two TDC hits: one from the muon, the other from the electron from decay muon. TDC information in areas of $17 \times 17$ channels around upstream and downstream track edges is checked for both top and side view. TDC hits within $100 \mathrm{~ns}$ from the first hit are ignored due to TDC dead time. There are two criterias for tagging decay particles corresponding to two cases.

Case 1: There are multiple TDC hits on both top and side view.

To avoid mis-identification by multiple TDC hits from noise, the same time interval between first hit and $i$ th hit are required for both top and side view. If the difference of the time interval on top and side is less than $20 \mathrm{~ns}$, they are identified as hits from decay particles (figure 4.4).

Case 2: There are multiple TDC hits only on one view.

The time of the $i$ th hit in one view and the time of the first hit in the other view are compared. If the time difference of those hits is less than 20 ns and the first hit which is not followed by second hit have more than 10 photoelectrons, it is also identified as a signal from decay particles (figure 4.5).

Muon lifetime is measured using data tagged as muon decay. Figure 4.6 shows the time distribution of muon decay sample. Horizontal axis is the time interval between primary TDC hits and secondary TDC hits that matched on top and side view. From the result of fitting, muon lifetime is $2.09 \mu \mathrm{s}$.

\section{$d E / d x$ Cut}

Protons have larger energy deposit compared to muons, charged pions and electrons for the same track length. Thus, protons can be identified using $d E / d x$ information. $d E / d x$ is calculated by total energy deposit along the track divided by the track length. Figure 4.7 shows track length v.s. $d E / d x$. Red points represent protons and black points represent other particles such as muons, charged pions and electrons. Line shows the cut for proton identification. Particles above the line are identified as protons. $d E / d x$ distributions for several track length are shown in figure 4.3.2, $d E / d x$ is calculated from 


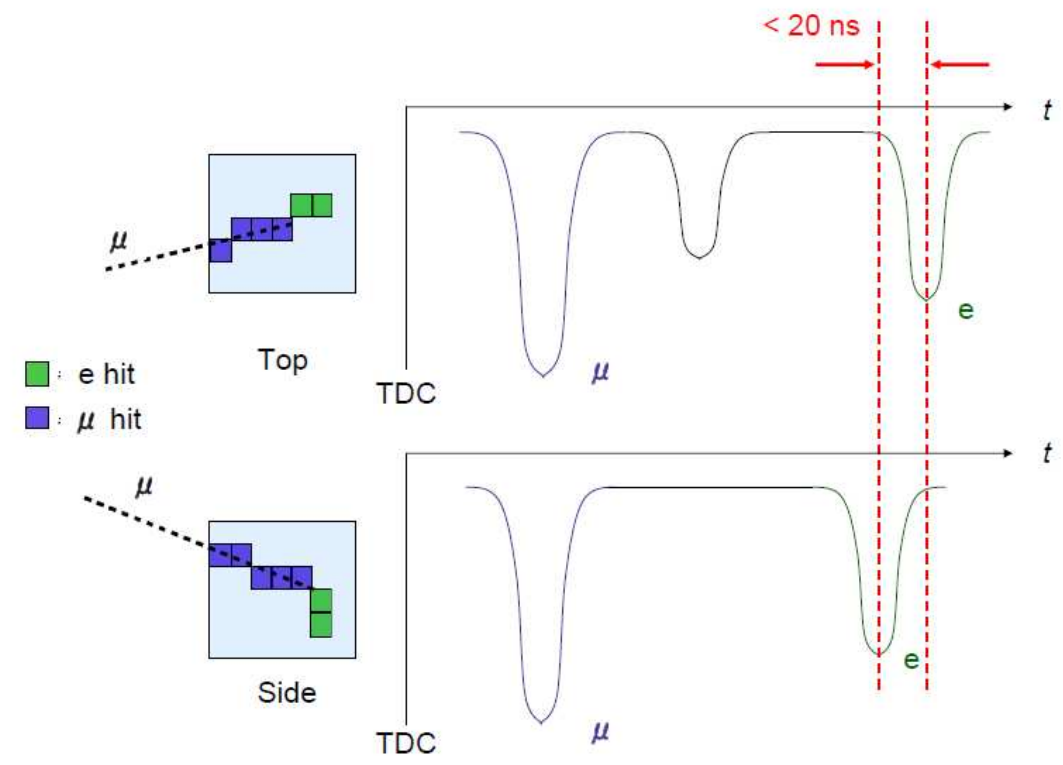

Figure 4.4: TDC hits from muon decay in top and side view. There are multiple TDC hits on both top and side view (case 1).

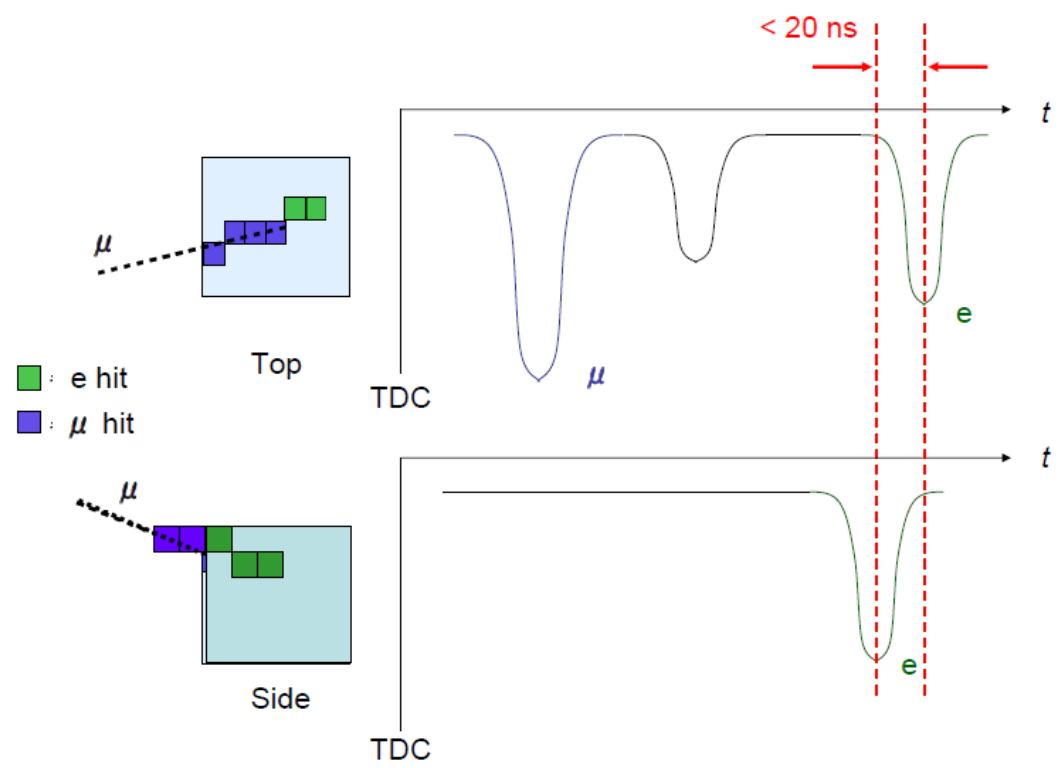

Figure 4.5: TDC hits from muon decay in top and side view. There are multiple TDC hits only on one view (case 2 ). 


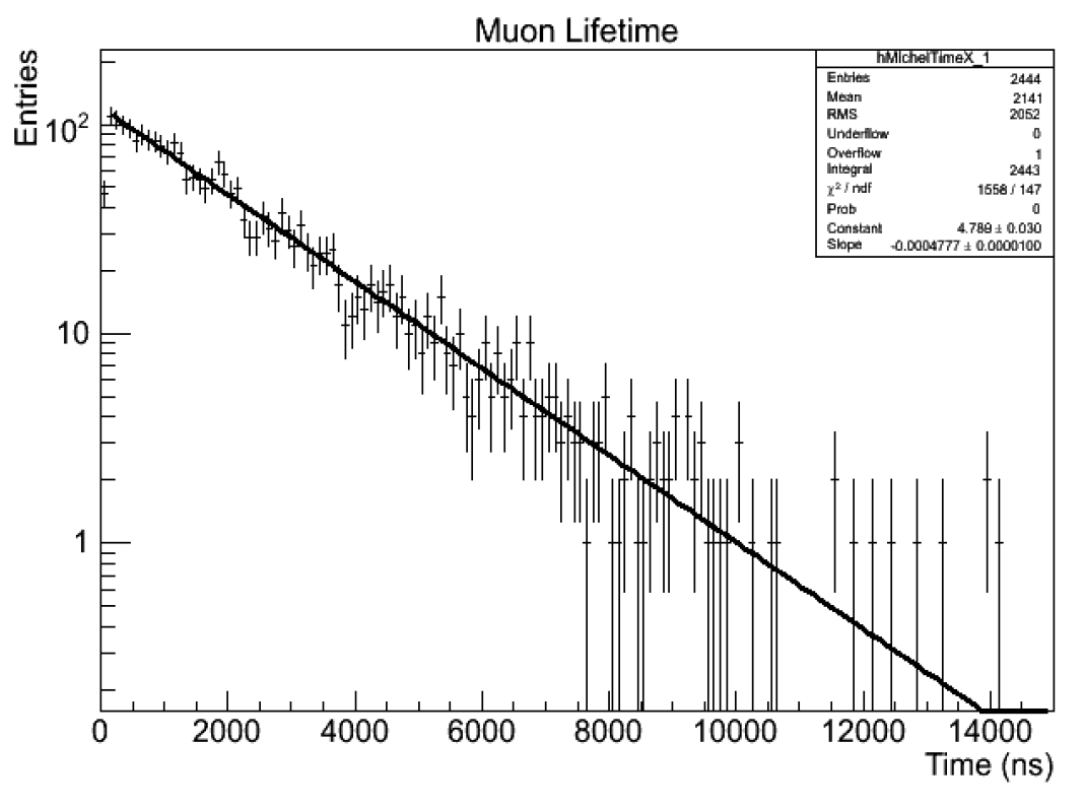

Figure 4.6: Time distribution of muon decay sample. Horizontal axis is the time interval between primary TDC hits and secondary TDC hits that matched on top and side view. 
total energy deposit of the track divided by the track length. The peak with large $d E / d x$ is from proton track while the peak with small $d E / d x$ is from muons, charged pions and electrons. Using this cut, protons are distinguished from other particles with $95 \%$ purity and $95 \%$ efficiency where efficiency is defined by number of protons above cut divided by total number of protons.

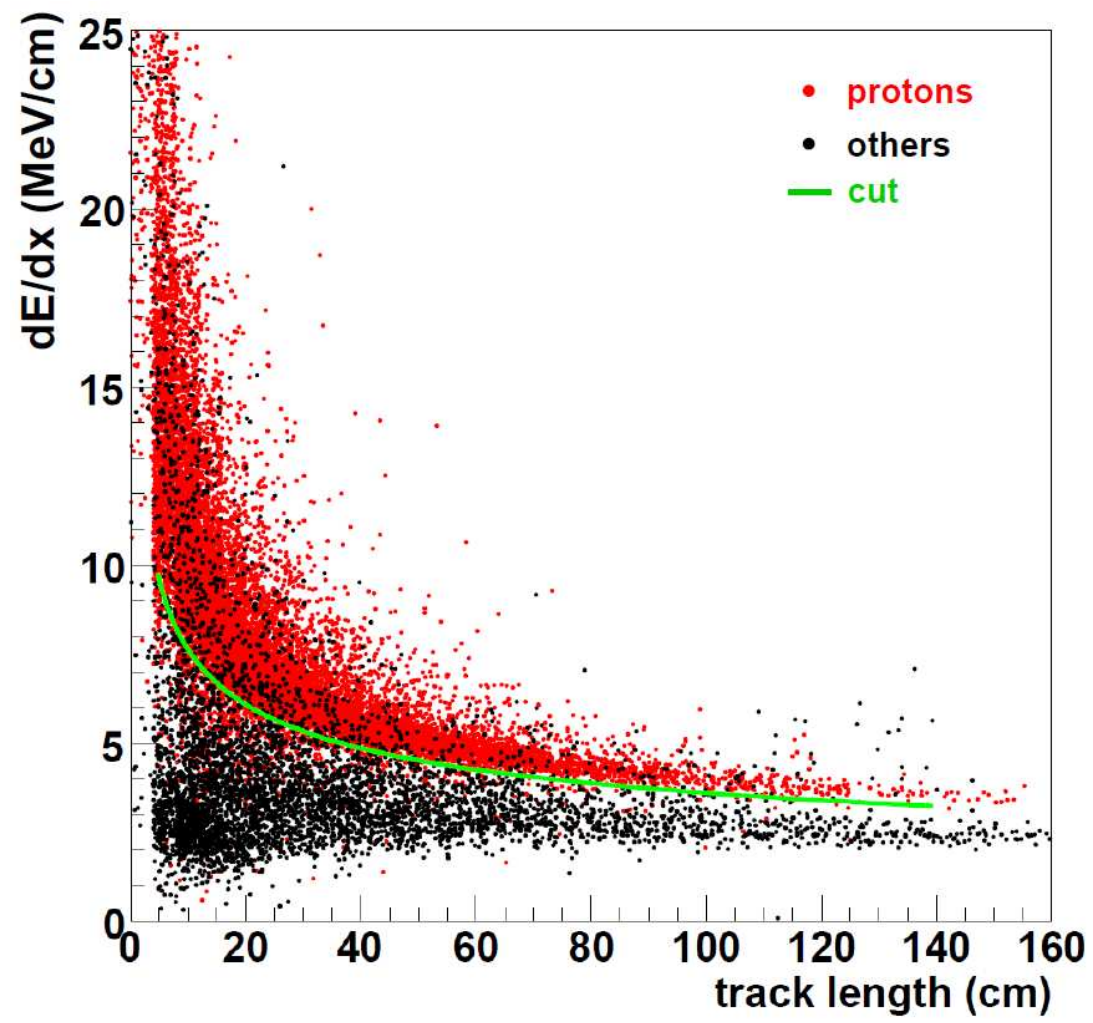

Figure 4.7: Track length v.s. $d E / d x$. Red points represent protons and black points represent other particles such as $\mu^{ \pm}, \pi^{ \pm}$and $e^{ \pm}$. Line shows the cut for proton identification. Particles above the line are identified as protons.

Angular distribution of NC elastic sample is shown in figure 4.9, $\theta$ is an angle of the track with respect to the $\mathrm{z}$ direction. Length distribution (left figure) and data/MC ratio (right figure) of $\mathrm{NC}$ elastic sample are shown in figure 4.10. There are data excesses in regions of the angle greater than $60^{\circ}$ and the track length shorter than $30 \mathrm{~cm}$. 

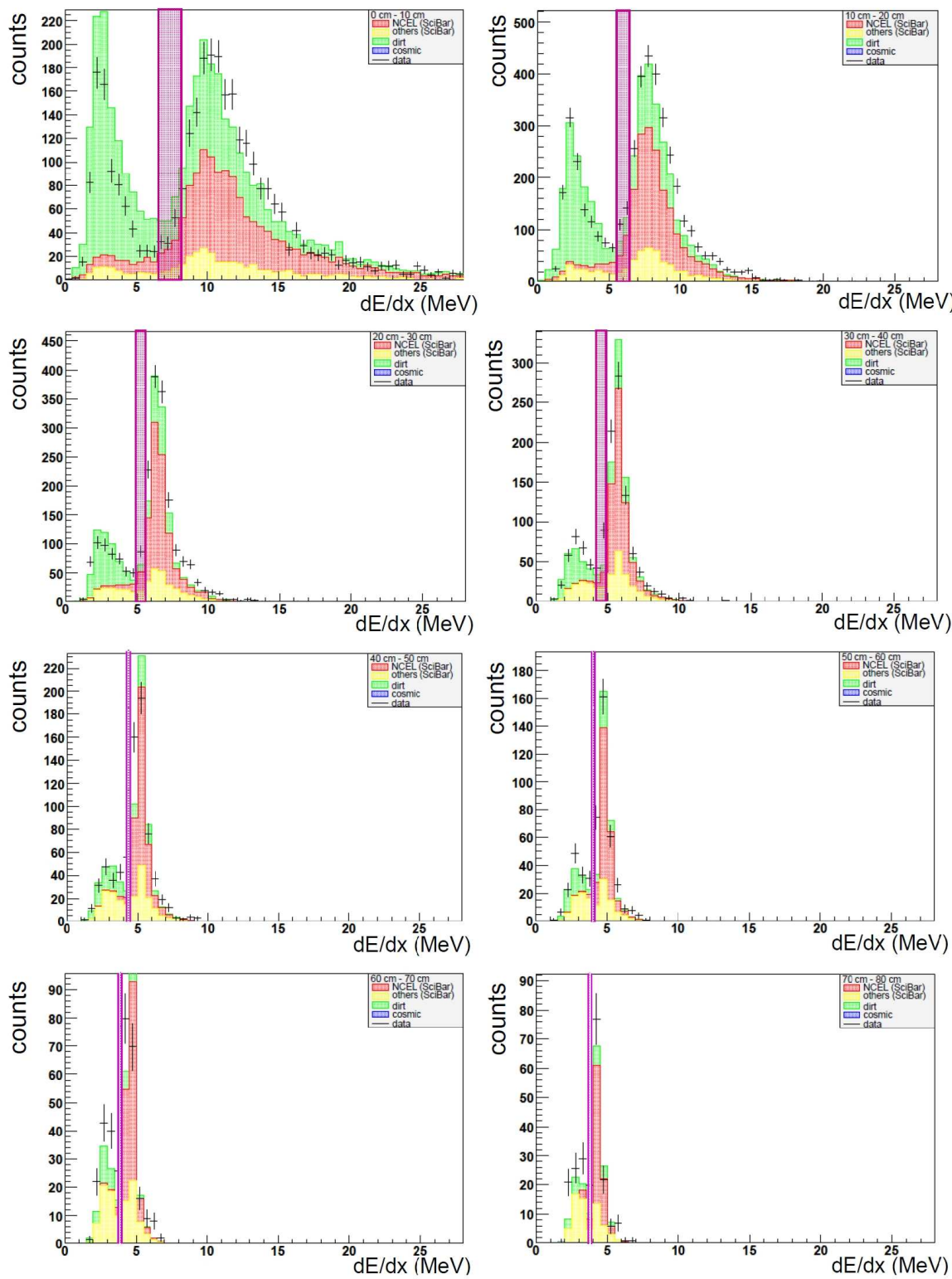

Figure 4.8: $d E / d x$ distributions for several different track lengths. The top left is $0-10 \mathrm{~cm}$, the bottom right is $70-80 \mathrm{~cm} . d E / d x$ is calculated from total energy deposit of the track divided by track length. The peak at large $d E / d x$ is from proton track while the peak at small $d E / d x$ is from other MIP particles. Data are compared to MC simulations. In these plots, dots with error bars is data and others are MC simulation. Red is NC elastic events, yellow is other interaction, and green is dirt events. 

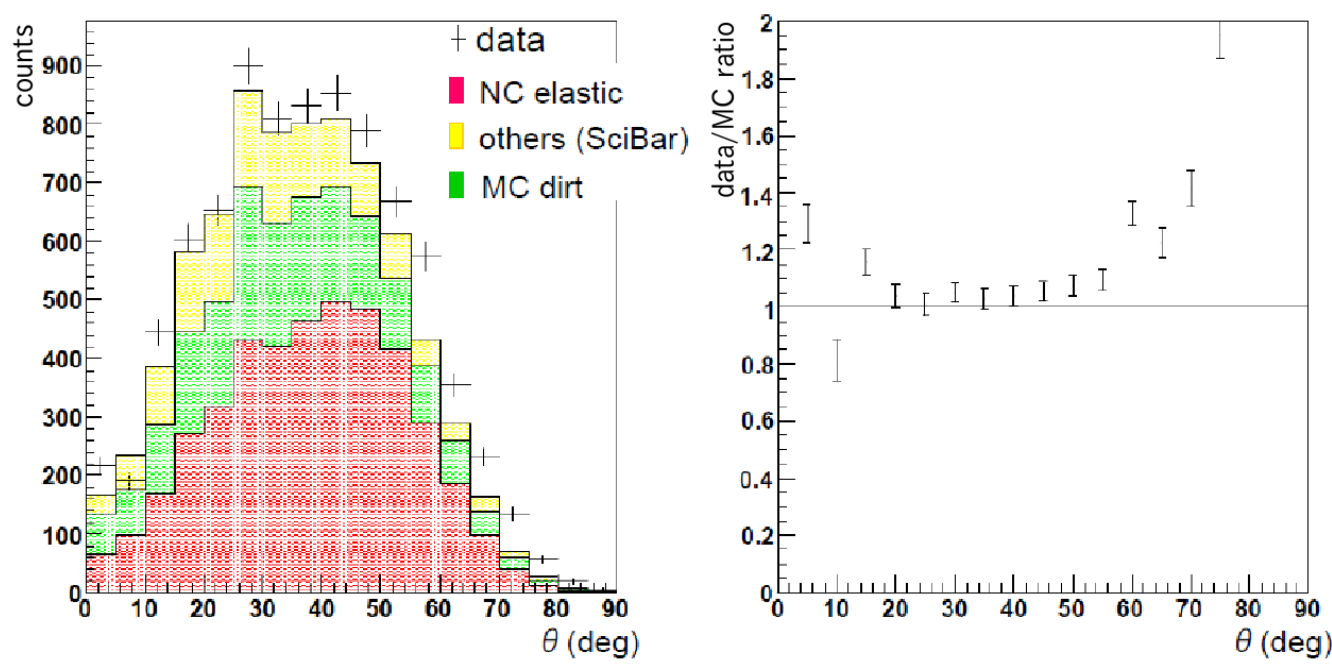

Figure 4.9: Angular distribution of $\mathrm{NC}$ elastic sample. $\theta$ is an angle of the track with respect to the $\mathrm{z}$ direction. Data are compared to $\mathrm{MC}$ simulation. Dots with error bars is data and others are MC simulation. Red is NC elastic events, yellow is other interaction, and green is dirt events. Right plot shows data/MC ratio. 

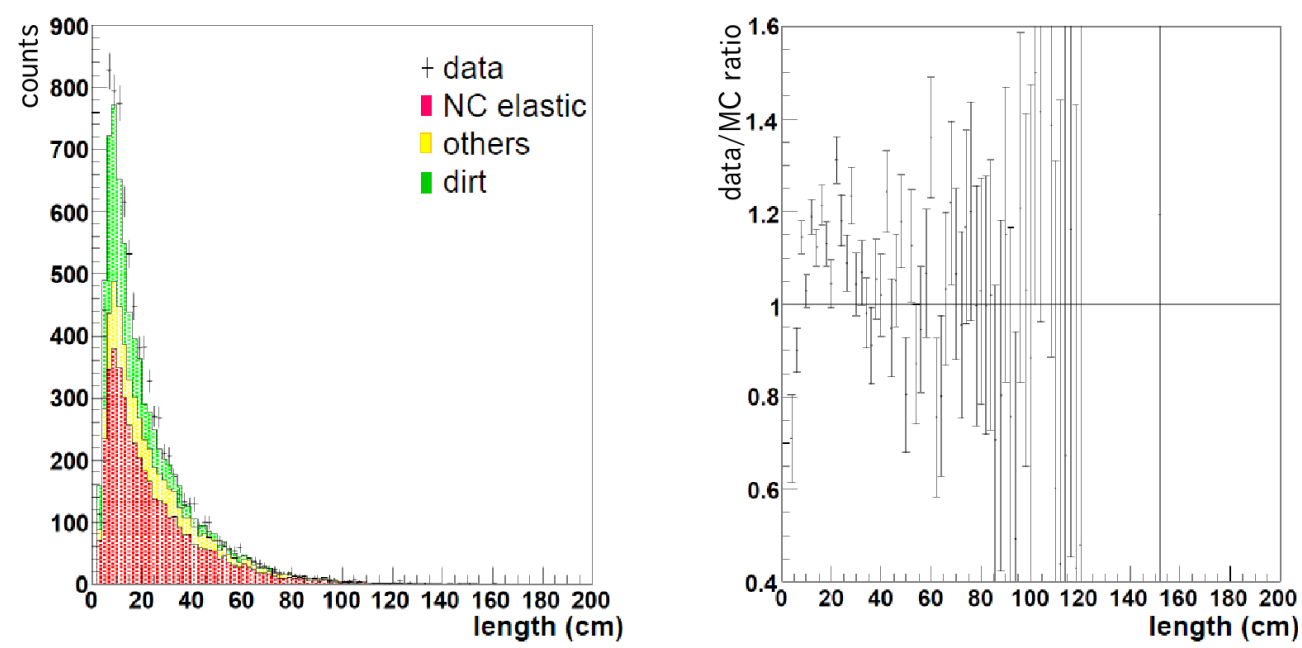

Figure 4.10: Length distribution (left figure) and data/MC ratio (right figure) of NC elastic sample. Data are compared to MC simulation. Dots with error bars is data and others are MC simulation. Red is NC elastic events, yellow is other interaction, and green is dirt events. Right plot shows data/MC ratio.

Proton kinetic energy is reconstructed from track length. To obtain kinetic energy from proton track length, a relationship between them are studied using true proton kinetic energy and true proton track length inside SciBar in MC simulation. Figure 4.11 shows true proton kinetic energy v.s. true proton track length. A line is a result of fitting. Distribution of proton kinetic energy is shown in figure 4.12. There is a data excess with proton kinetic energy less than $0.2 \mathrm{GeV}$ which corresponds to proton track length of $30 \mathrm{~cm}$ and $Q^{2}$ of about $0.4(\mathrm{GeV} / \mathrm{c})^{2}$.

There is a possibility that tracking algorithm works differently for data and $\mathrm{MC}$ when a track is short. Currently the discrepancy between data and $\mathrm{MC}$ in the region of proton kinetic energy less than $0.2 \mathrm{GeV}$ is being studied. In this region, there is also a large contribution of dirt events. Data with proton kinetic energy greater than $0.2 \mathrm{GeV}$ are used for further analysis. 


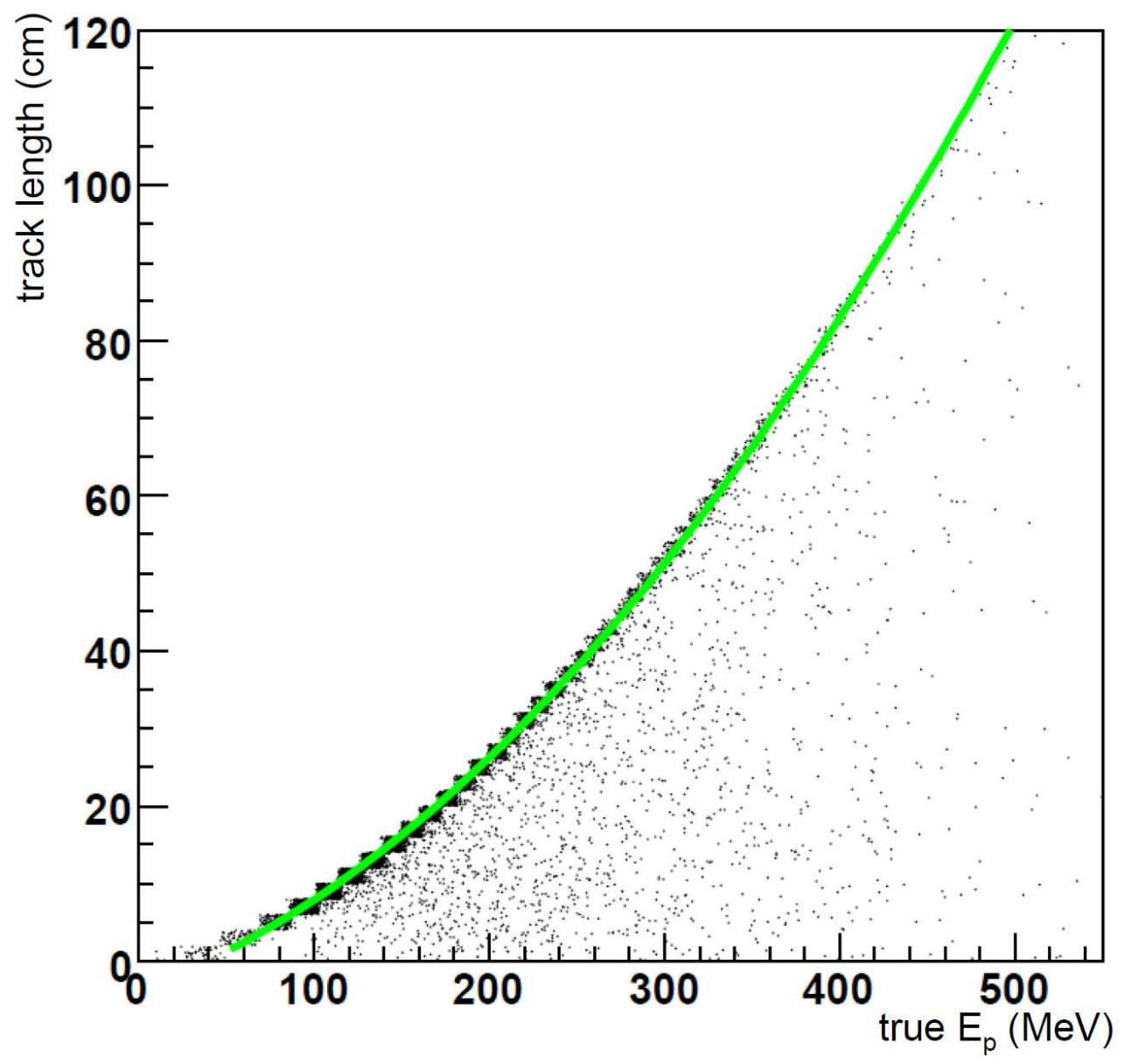

Figure 4.11: True proton kinetic energy v.s. true proton track length from $\mathrm{MC}$ simulation. A line is a result of fit. 


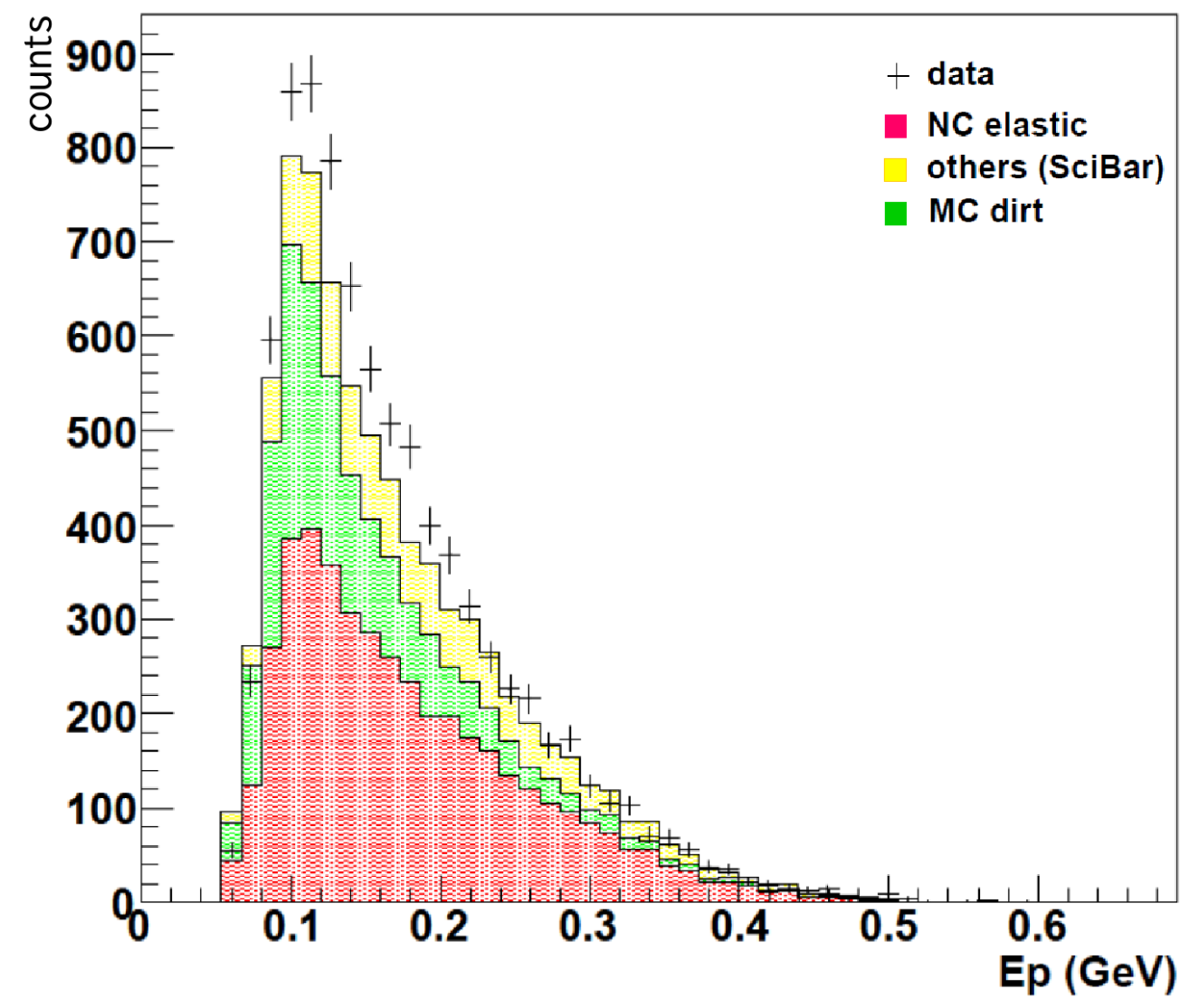

Figure 4.12: Distribution of proton kinetic energy. Proton kinetic energy is reconstructed from track length. Data are compared to MC simulation. 


\subsection{Background Estimation}

There are two major backgrounds in the NC elastic sample. One is from NC pion production without electromagnetic shower caused by gammas from decay neutral pion. The other background is from protons recoiled by dirt neutrons. Both of backgrounds are estimated using MC simulation. NC pion production candidates are, in usual case, events with two tracks of electromagnetic shower caused by two gammas from decay neutral pion. NC pion production at SciBooNE is now being studied [67]. In this analysis, $\mathrm{NC}$ pion production candidates shows good agreement between data and MC in number of event, geometrical information of the signal and reconstructed mass of neutral pions. Current MC simulation for NC pion production can be used for background estimation.

Dirt MC simulation was checked whether tuning is needed or not. There are more dirt events in upstream part of SciBar than downstream part. Figure 4.13 shows distributions of vertex position along $z$ axis v.s. track length of data (left), SciBar MC (middle) and dirt MC simulation (right). SciBar is divided into 8 bins along $z$ direction to compare number of events at upstream part with that of downstream part. Fifth bin is taken as a reference and is compared with first, second and third bins.
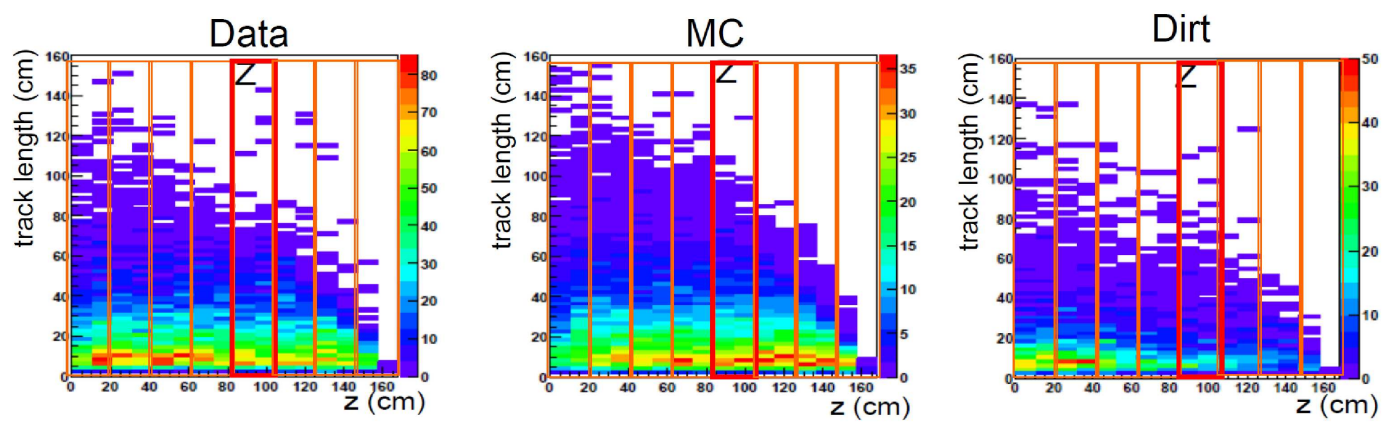

Figure 4.13: Vertex position along $z$ axis v.s. track length of data (left), Monte-Carlo simulation in SciBar (middle) and dirt Monte-Carlo simulation (right). SciBar is divided into 8 bins along $z$ direction to compare number of events at upstream part with that of downstream part. Fifth bin is taken as a reference and compared with first, second and third bins.

Track length distributions of (upstream bins)-(fifth bin) are shown in 
figure 4.14. Data and sum of SciBar MC and dirt MC are compared. Data agrees with MC simulation within statistic error.
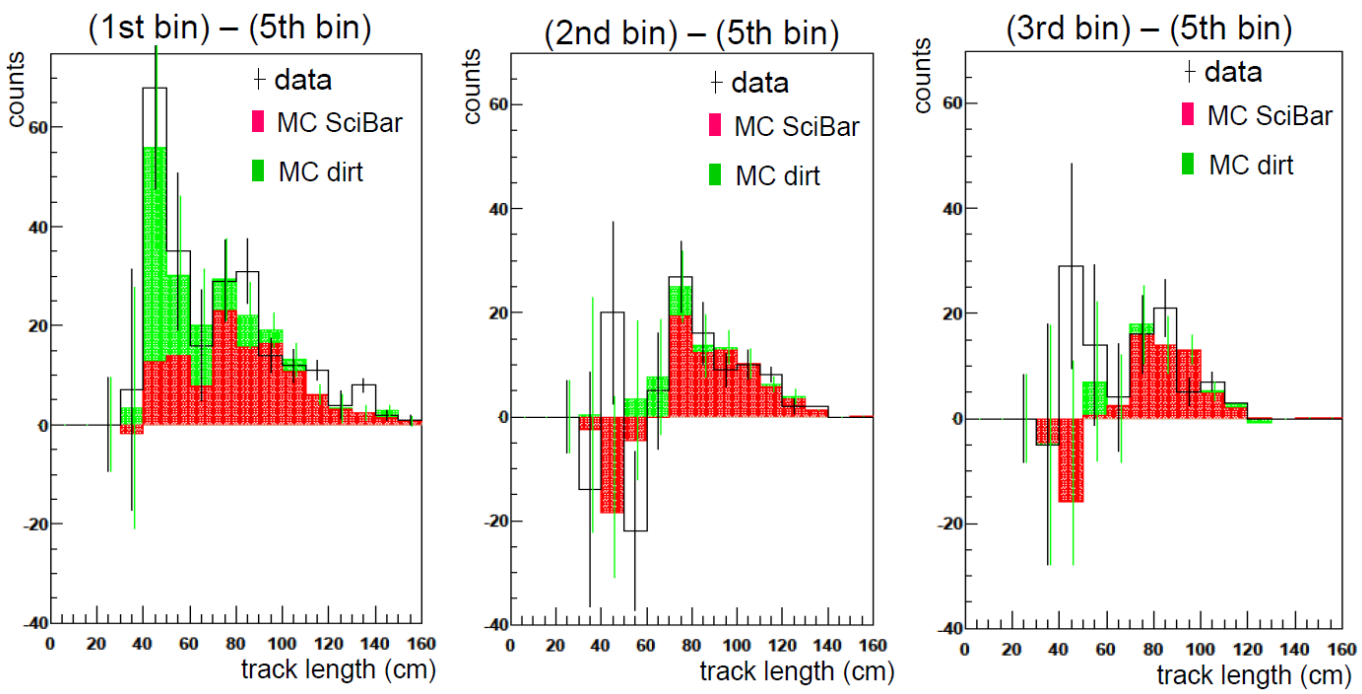

Figure 4.14: Track length distributions of (upstream bins)-(fifth bin). Data agree with Monte-Carlo simulation within statistic error.

Particles which have roots in dirt events and make NC elastic scatteringlike track in SciBar are shown in Table4.1. 95\% of the dirt background is proton track. Fractions of the neutrino interactions in the dirt which make proton track in SciBar are shown in Table4.2. Proton tracks from dirt events are mainly recoiled by neutrons from the dirt. Neutrino-neutron NC elastic scattering $(\nu n \rightarrow \nu n)$ in the dirt have the largest contribution to the dirt background. 


\begin{tabular}{|c|c|}
\hline particle & number of events \\
\hline \hline proton & 1814 \\
\hline electron & 64 \\
\hline muon & 4 \\
\hline pion & 19 \\
\hline total & 1901 \\
\hline
\end{tabular}

Table 4.1: Particles which have roots in dirt events and make NC elastic scattering-like track in SciBar.

\begin{tabular}{|c|c|}
\hline interaction & fraction $(\%)$ \\
\hline \hline NC elastic $(\nu p \rightarrow \nu p)$ & 3 \\
\hline NC elastic $(\nu n \rightarrow \nu n)$ & 30 \\
\hline NC pion production & 13 \\
\hline CC elastic $\left(\nu n \rightarrow \mu^{-} p\right)$ & 16 \\
\hline CC pion production & 27 \\
\hline
\end{tabular}

Table 4.2: Fractions of the neutrino interactions in the dirt which make a proton track in SciBar.

\subsection{Event Selection Summary}

Summary of the NC elastic event selection is shown in Table 4.1. MC (signal) is $\nu p \rightarrow \nu p$ and $\nu n \rightarrow \nu n$ in MC simulation. MC (B. G.) is a total of non-NC elastic events in SciBar and all dirt events. After event selection, there are 8441 events in data and 7675 events in MC.

\begin{tabular}{|c||c|c|c|c|}
\hline event selection & data & MC (signal) & MC (B. G.) & purity (\%) \\
\hline \hline total & 1877675 & 34962 & 161296 & 21.7 \\
\hline veto & 123501 & 27962 & 96341 & 22.5 \\
\hline 1 track & 18325 & 5200 & 16415 & 24.7 \\
\hline decay particle rejection & 13917 & 5017 & 7739 & 39.3 \\
\hline$d E / d x$ cut & 8441 & 4340 & 3335 & 56.5 \\
\hline
\end{tabular}

Table 4.3: Event selection summary table of the NC elastic event selection. 


\subsection{Detector Unfolding}

Proton kinetic energy distribution after background subtraction is shown in figure 4.15. Bin width is changed considering the bin width of $Q^{2}$ distribution which is reconstructed from proton kinetic energy.

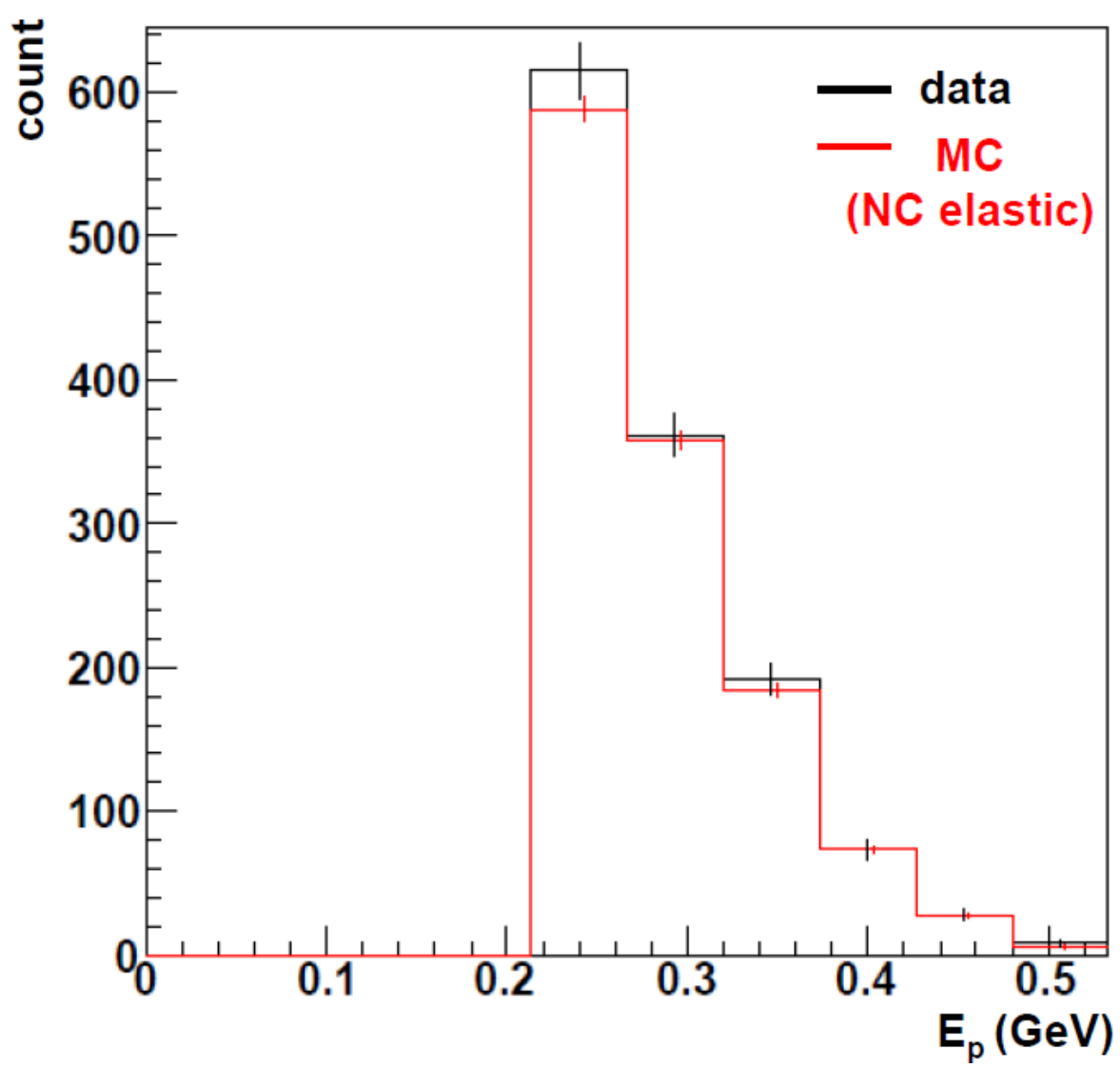

Figure 4.15: Proton kinetic energy distribution after background subtraction.

Proton kinetic energy is smeared by track length resolution of the detector. Detector unfolding is applied to the $\mathrm{NC}$ elastic sample after background subtraction. Figure 4.16 shows distribution of true proton kinetic energy v.s. proton kinetic energy reconstructed from measured track length using tracking algorithm (left figure). Both quantities are from MC simulation. From this relationship, detector unfolding matrix was made (right figure). 

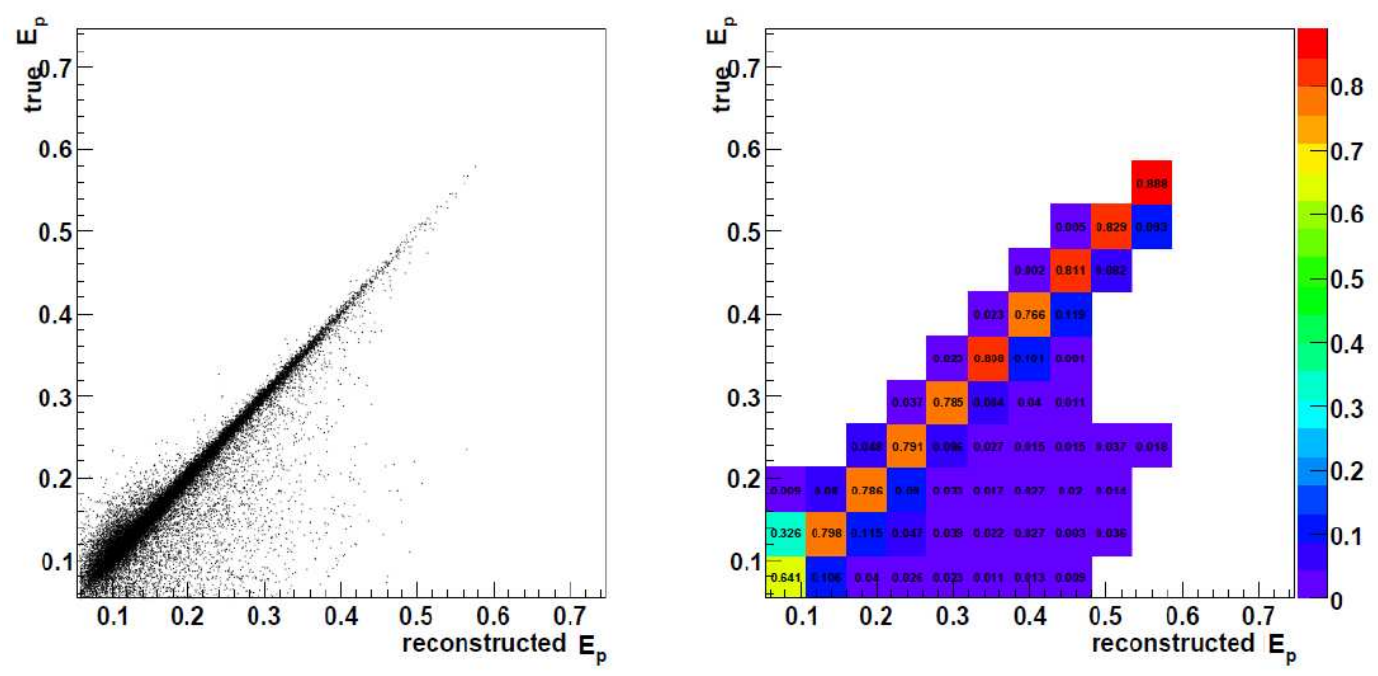

Figure 4.16: Proton kinetic energy reconstructed from track length using Monte-Carlo simulation v.s. proton kinetic energy reconstructed from measured track length using tracking algorithm (left figure). Both quantities are from MC simulation. From this relationship, unfolding matrix was made (right figure). 
Detector unfolding matrix $U_{\text {det }}$ is defined as

$$
U_{\text {det }}=\left(\begin{array}{cccccccccc}
0.64 & 0.11 & 0.04 & 0.03 & 0.02 & 0.01 & 0.01 & 0.01 & 0 & 0 \\
0.33 & 0.80 & 0.12 & 0.05 & 0.04 & 0.03 & 0 & 0 & 0 & 0 \\
0.01 & 0.08 & 0.79 & 0.09 & 0.03 & 0.02 & 0.03 & 0.02 & 0.01 & 0 \\
0 & 0 & 0.05 & 0.79 & 0.09 & 0.03 & 0.02 & 0.02 & 0.04 & 0.02 \\
0 & 0 & 0 & 0.04 & 0.79 & 0.08 & 0.04 & 0.01 & 0 & 0 \\
0 & 0 & 0 & 0 & 0.02 & 0.81 & 0.10 & 0 & 0 & 0 \\
0 & 0 & 0 & 0 & 0 & 0.02 & 0.77 & 0.12 & 0 & 0 \\
0 & 0 & 0 & 0 & 0 & 0 & 0 & 0.81 & 0.08 & 0 \\
0 & 0 & 0 & 0 & 0 & 0 & 0 & 0.01 & 0.83 & 0.09 \\
0 & 0 & 0 & 0 & 0 & 0 & 0 & 0 & 0 & 0.89
\end{array}\right)
$$

Number of events in the $i$ th bin of true proton kinetic energy is obtained using the unfolding matrix as follows:

$$
n_{i}^{\text {true }}=\sum_{j} u_{j i} n_{j}^{r e c}
$$

where $j$ is the bin of reconstructed proton kinetic energy, $n_{j}^{r e c}$ is the number of events in $j$ th bin, and $u_{j i}$ is the $(j, i)$ element of the unfolding matrix.

Figure 4.17 shows proton kinetic energy distribution after detector unfolding.

\subsection{Efficiency Correction}

After detector unfolding, proton kinetic energy distribution is still biased by detector acceptance and tracking efficiency. To obtain unbiased distribution, correction for detection efficiency is needed. Detection efficiency is defined using $\mathrm{MC}$ for each true proton kinetic energy bin as number of events in NC elastic sample divided by total number of events generated in SciBar. Figure 4.18 shows the detection efficiencies for each proton kinetic energy bin.

Yield of $i$ th bin $Y_{i}$ is written as

$$
Y_{i}=n_{i}^{\text {true }} \epsilon_{i}
$$

where $\epsilon_{i}$ is the detection efficiency for $i$ th true proton kinetic energy bin.

Proton kinetic energy distribution after efficiency correction is shown in figure 4.19. 


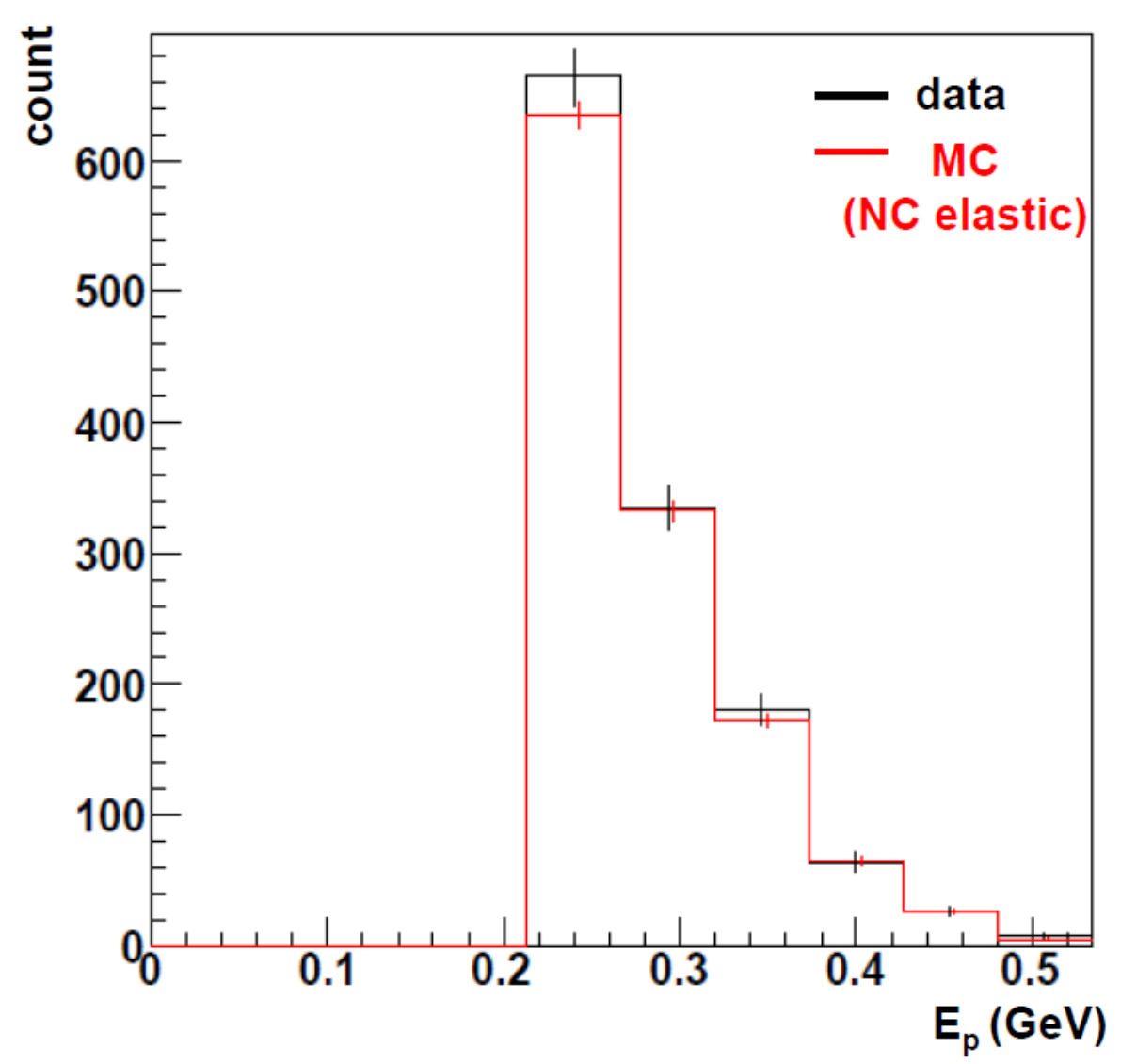

Figure 4.17: Proton kinetic energy distribution after detector unfolding. Data are compared to MC simulation. 


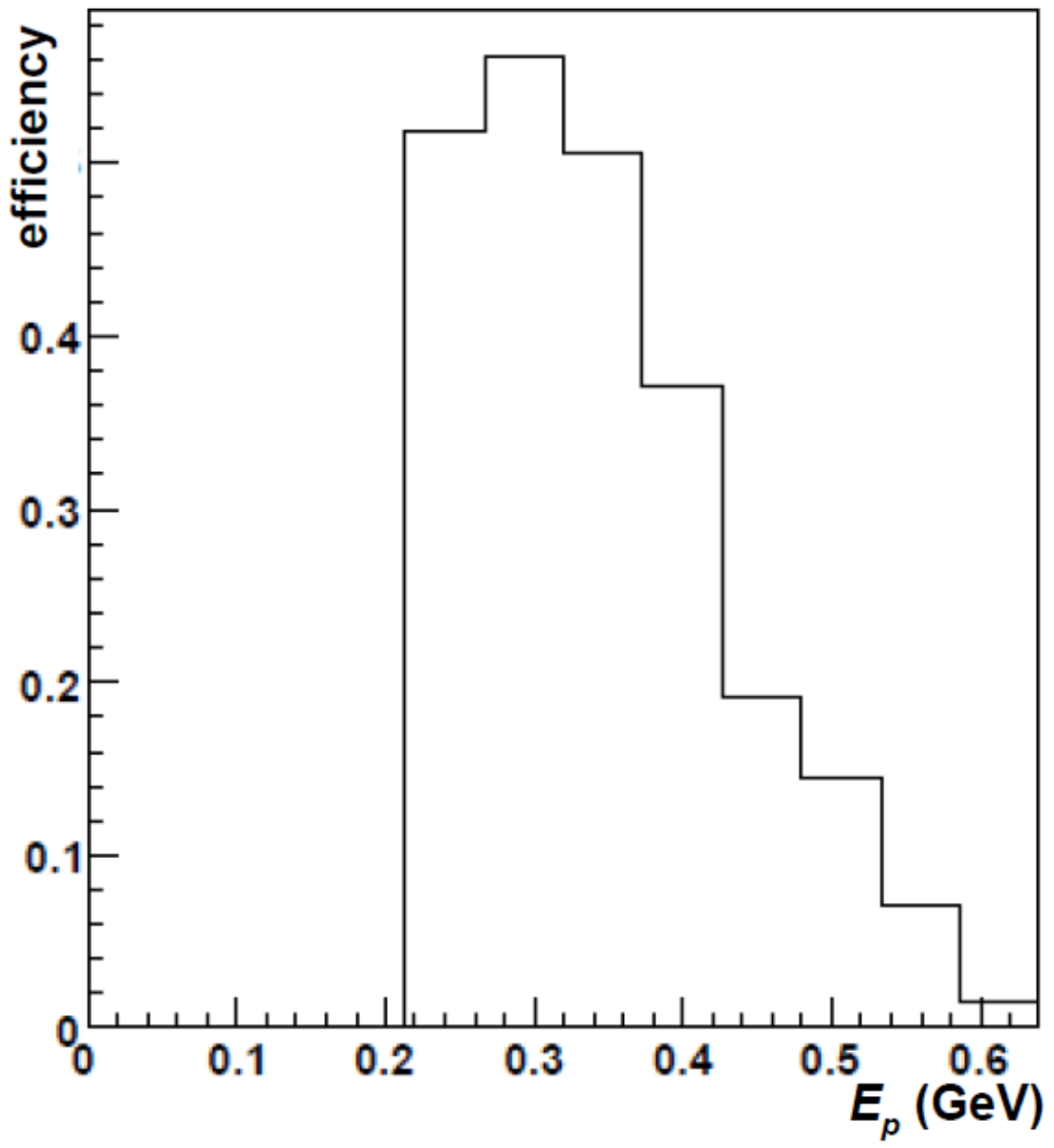

Figure 4.18: MC true proton kinetic energy v.s. detection efficiency. 


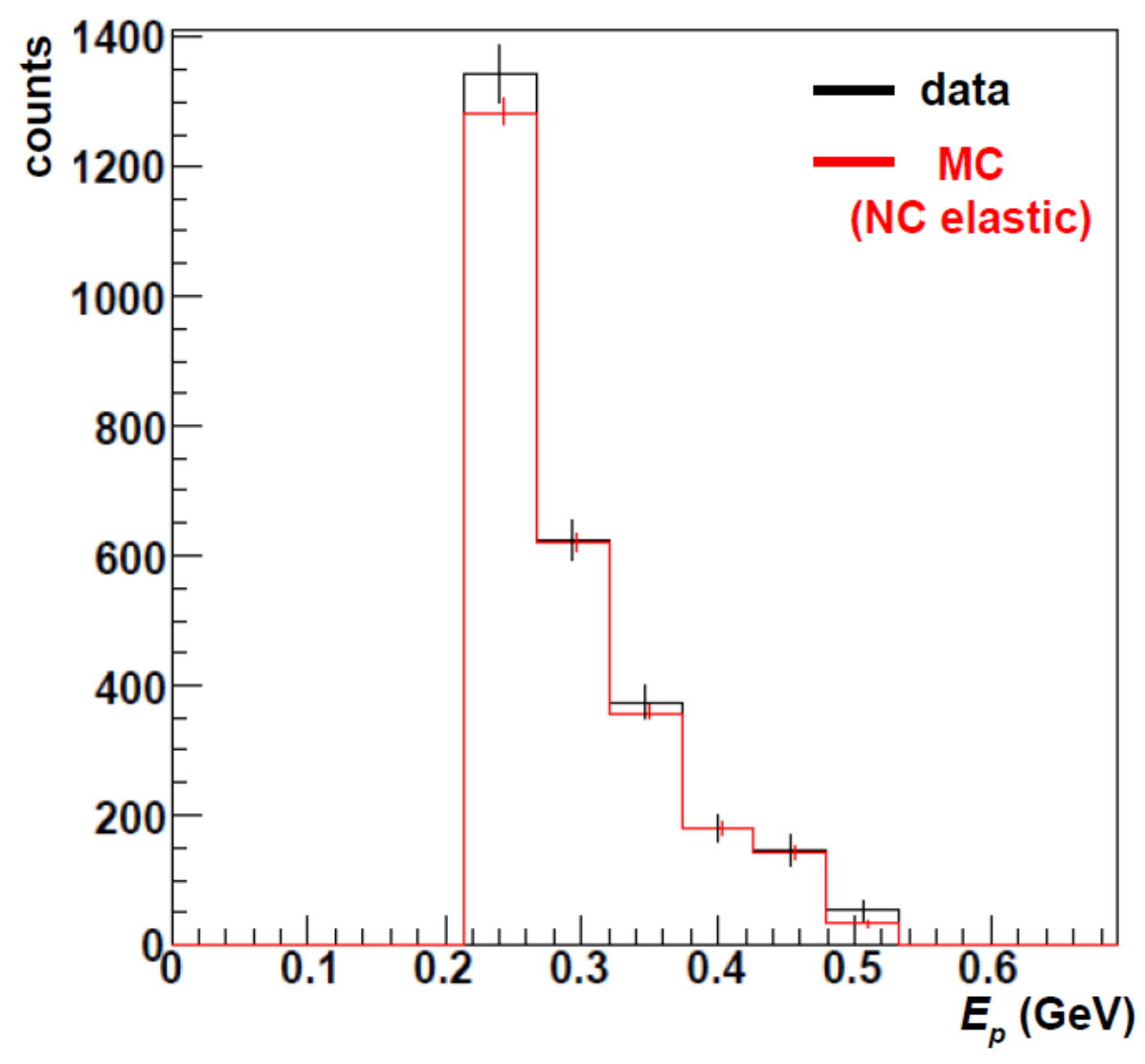

Figure 4.19: Proton kinetic energy distribution after efficiency correction. Data are compared to MC simulation. 


\subsection{Cross Section}

Cross section is evaluated from the yield, the number of neutrinos that entered the detector, and the number of targets:

$$
\frac{d \sigma_{i}}{d E_{p}}=\frac{Y_{i} /\left(\Delta E_{p}\right)}{N_{\text {POT }} N_{\text {target }} \int \phi_{\nu}\left(E_{\nu}\right) d E_{\nu}}
$$

where $d \sigma_{i} / d E_{p}$ is $i$ th $E_{p}$ bin of the differential cross section. $Y_{i}$ is a yield of $i$ th bin after several corrections. $\Delta E_{p}$ is the bin width of the $E_{p}$ distribution. $N_{P O T}$ and $N_{\text {target }}$ are the number of proton on beryllium target and the number of nucleons in SciBar FV. $\phi_{\nu}\left(E_{\nu}\right)$ is the neutrino flux. For this analysis, data sample of $9.9 \times 10^{19}$ POT are used. Neutrino flux cannot be measured directly. It is evaluated using neutrino beam MC simulation. Integrated flux $\int \phi_{\nu}\left(E_{\nu}\right) d E_{\nu}$ is determined to be $1.91 \times 10^{-8}\left(1 /\left(\mathrm{cm}^{2} \cdot \mathrm{POT}\right)\right)$ using beam MC simulation. $N_{\text {target }}$ is calculated by $N_{\text {target }}=N_{A} M_{S B}$ where $N_{A}$ is Avogadro's number, $M_{S B}=9.38 \times 10^{6} \mathrm{~g}$ is the mass of SciBar FV.

Differential cross section as a function of proton kinetic energy is shown in figure 4.20, Error bars include statistical error only. Systematic error is estimated in Section 4.9. 


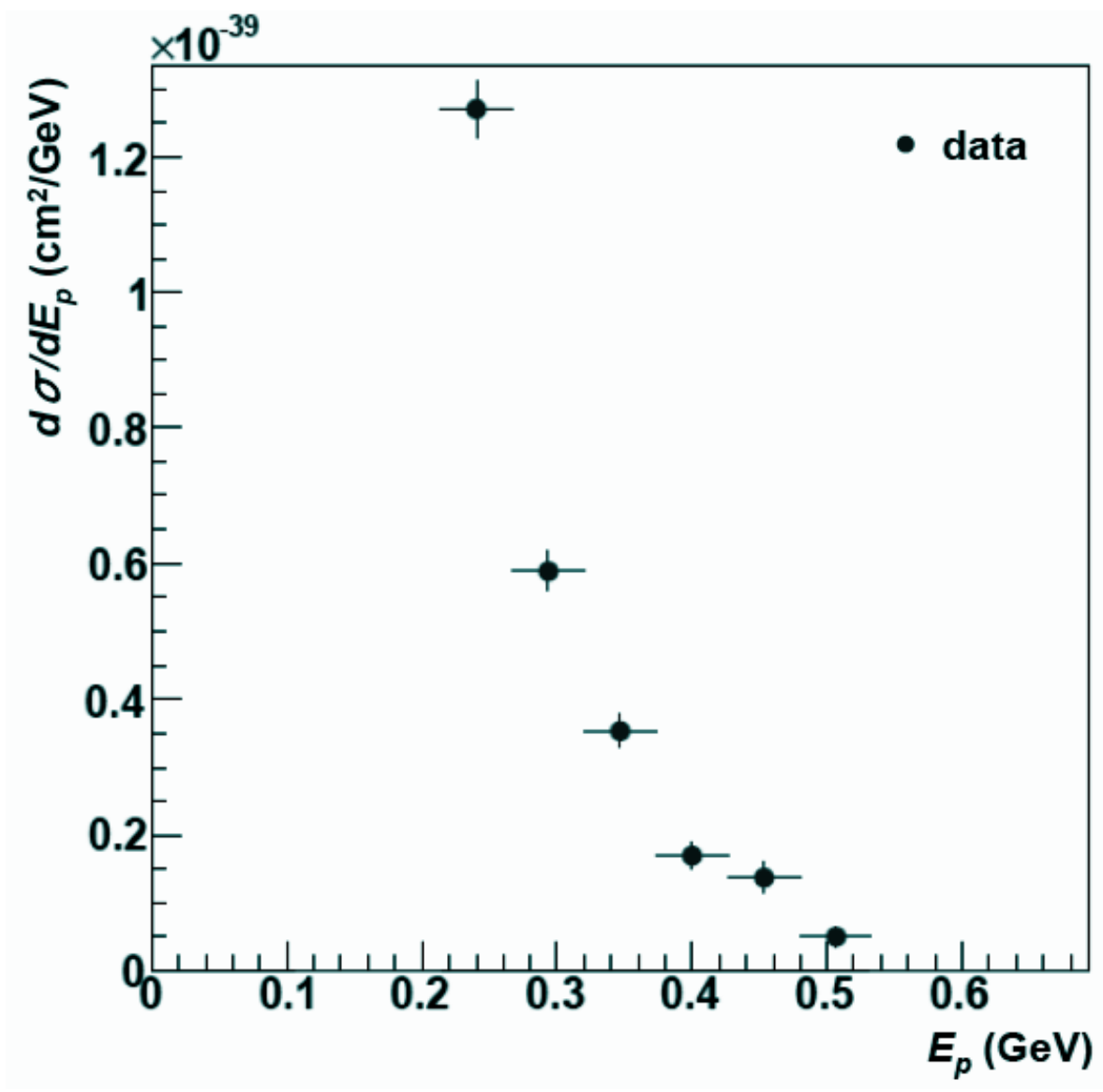

Figure 4.20: Differential cross section as a function of proton kinetic energy. 


\subsection{Systematic Uncertainty}

\subsubsection{Neutrino Beam}

The sources of the systematic uncertainty in the neutrino beam mainly come from secondary particle production, hadronic interactions, horn magnetic field and number of protons on target. Details are written in reference [20].

\section{Secondary Particle Production}

Secondary particles such as pions and kaons from the proton-beryllium target interaction is simulated by the beam simulation using two models. The Sanford-Wang model is used for $\pi^{+}, \pi^{-}$and $K^{0}$ productions. The Feynman scaling model is used for $K^{+}$production. The Sanford-Wang parameterization and the Feynman scaling parameterization are discussed in Section 3.6. The uncertainty in the fitted parameters is evaluated by varying the parameters of the models.

\section{Hadronic Interactions:}

The uncertainty in the hadronic interactions such as proton-beryllium interactions and the probability for mesons to survive possible hadronic interactions in the target or horn and decay to produce neutrinos. Uncertainties in the cross sections of these interactions affect both the rate and shape of the flux.

\section{Horn Magnetic Field:}

The uncertainty in the modeling of the current within the inner cylinder in the magnetic focusing horn is taken into account. The horn current has uncertainty of $\pm 1 \mathrm{kA}$.

\section{Protons On Target:}

The uncertainty in the measurement of the number of protons delivered to the target is evaluated to be $\sim 2 \%$. This uncertainty is considered as a normalization error of the cross section.

The change in the neutrino flux due to these uncertainties is calculated by using random parameter vectors and weighting each event by a factor corresponding to the variation of the yield of the parent meson with the given 
momentum and angle. 1,000 parameter vector sets are used for the calculation. Systematic uncertainty in the $\mathrm{NC}$ elastic cross section due to the uncertainty in the neutrino beam is shown in Figure 4.21,

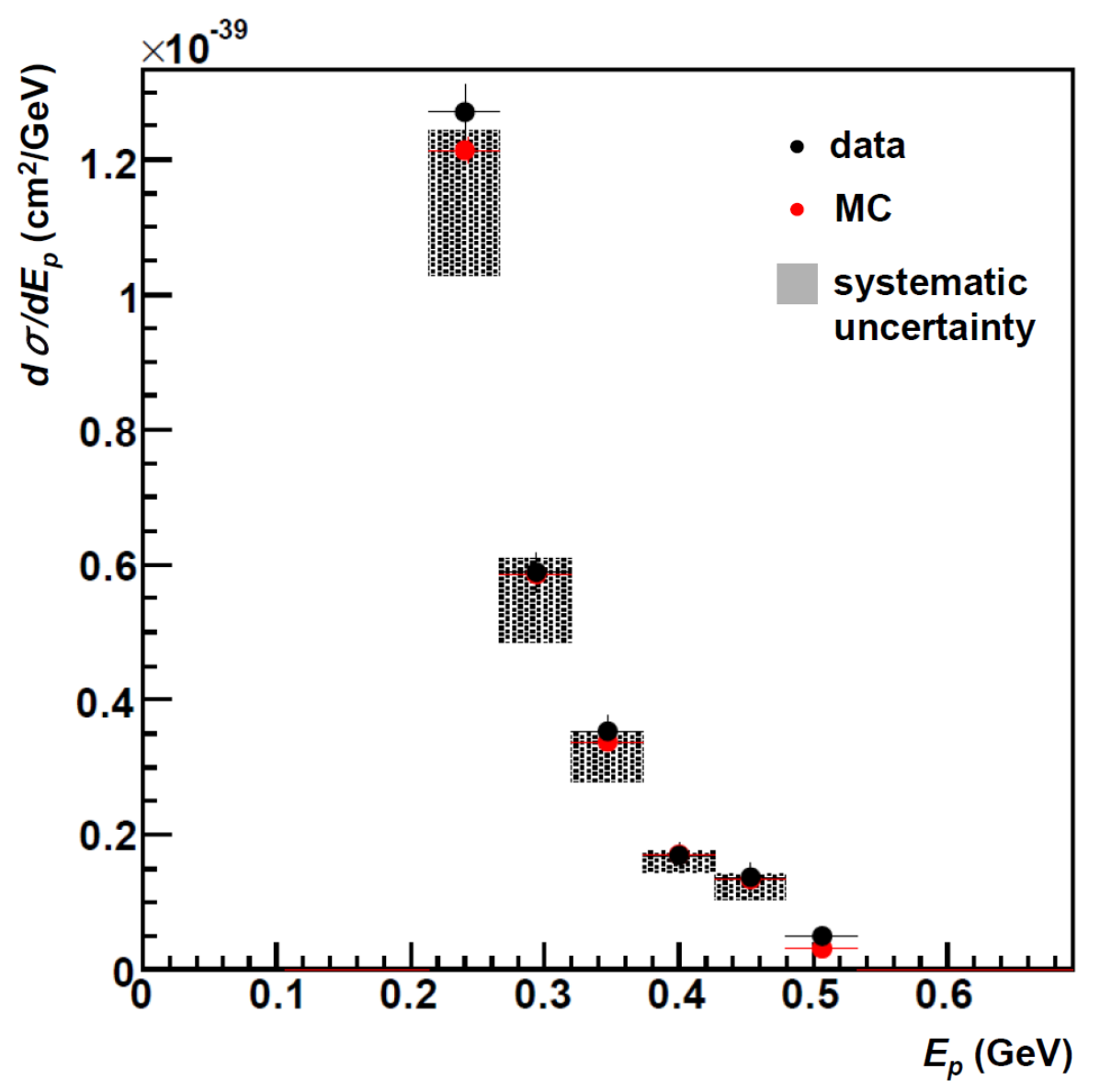

Figure 4.21: Systematic uncertainty in the NC elastic cross section due to the uncertainty in the neutrino beam.

\subsubsection{Neutrino Interaction}

Axial vector mass $M_{A}$ is set to $1.1 \mathrm{GeV} / c^{2}$. The uncertainty in $M_{A}$ is estimated to be approximately $\pm 0.1 \mathrm{GeV} / c^{2}$ based on results from $\mathrm{K} 2 \mathrm{~K}$ [68] and MiniBooNE [69] experiments. $M_{A}$ is varied to $1.2 \mathrm{GeV} / c^{2}$ considering 
the results from these experiments and the other results from the past experiments [70] to estimate the systematic uncertainty due to $M_{A}$ value. Figure 4.22 shows the systematic uncertainty due to $M_{A}$ uncertainty.

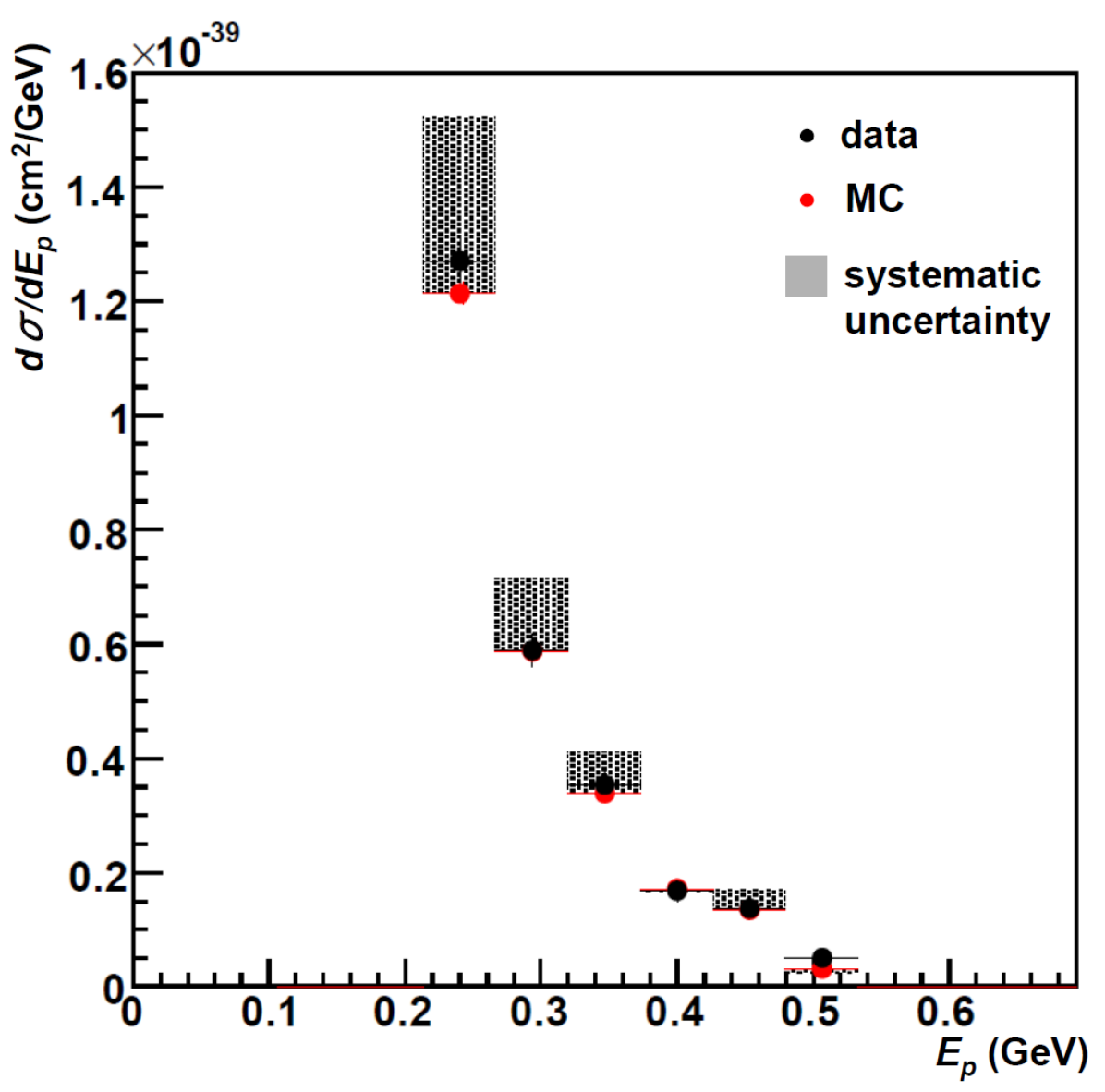

Figure 4.22: The systematic uncertainty due to $M_{A}$ uncertainty.

\subsubsection{Detector Response}

The uncertainty in detector response comes from the measurement error of the cross talk, the scintillator quenching, single photoelectron resolution and the hit threshold for track reconstruction. Since only tracks with large energy deposit are used for $\mathrm{NC}$ elastic analysis, the uncertainty in the crosstalk and 
the scintillator quenching are dominant.

\section{Crosstalk}

Crosstalk of the MAPMT is measured for an adjacent channel, a diagonal channel and a next-to-next channel. The amount of the crosstalk for an adjacent channel and an diagonal channel are $3.15 \%$ and $0.7 \%$, respectively. The amount of the crosstalk for a next-to-next channel is from $0.1 \%$ to $0.3 \%$. The absolute error of the crosstalk to the adjacent channel is estimated to be $0.4 \%$.

\section{Scintillator Quenching}

Scintillator quenching is simulated using Birk's law 64]. Birk's constant of the SciBar scintillator is measured to be $0.0208 \pm 0.0003$ (stat.) \pm 0.0023 (sys.) $\mathrm{cm} / \mathrm{MeV}$ [65].

The uncertainty in NC elastic cross section due to the crosstalk is estimated by varying crosstalk value to $2.75 \%$ and $3.55 \%$ in the simulation. The uncertainty in NC elastic cross section due to the scintillator quenching is estimated by varying the Birk's constant by $\pm 0.0023 \mathrm{~cm} / \mathrm{MeV}$ in the simulation.

Figure 4.23 shows the systematic uncertainty due to the uncertainty in the detector response.

\subsubsection{Total Systematic Uncertainty}

All the systematic uncertainties discussed in this section are combined. Each systematic uncertainty is independent to others. Figure 5.1 shows total systematic uncertainty in $\mathrm{NC}$ elastic scattering cross section as a function of proton kinetic energy. 


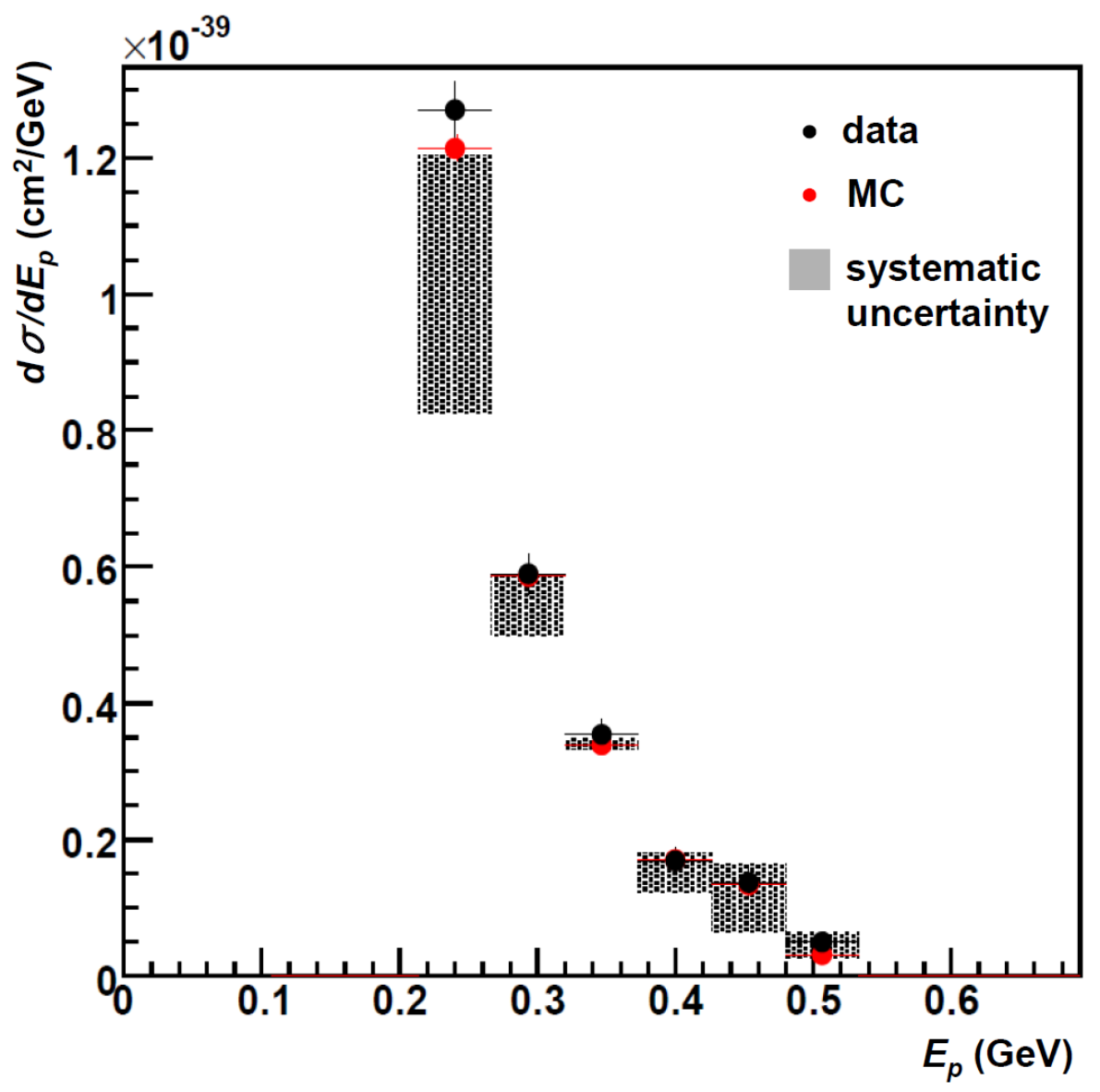

Figure 4.23: The systematic uncertainty due to the uncertainty in the detector response. 


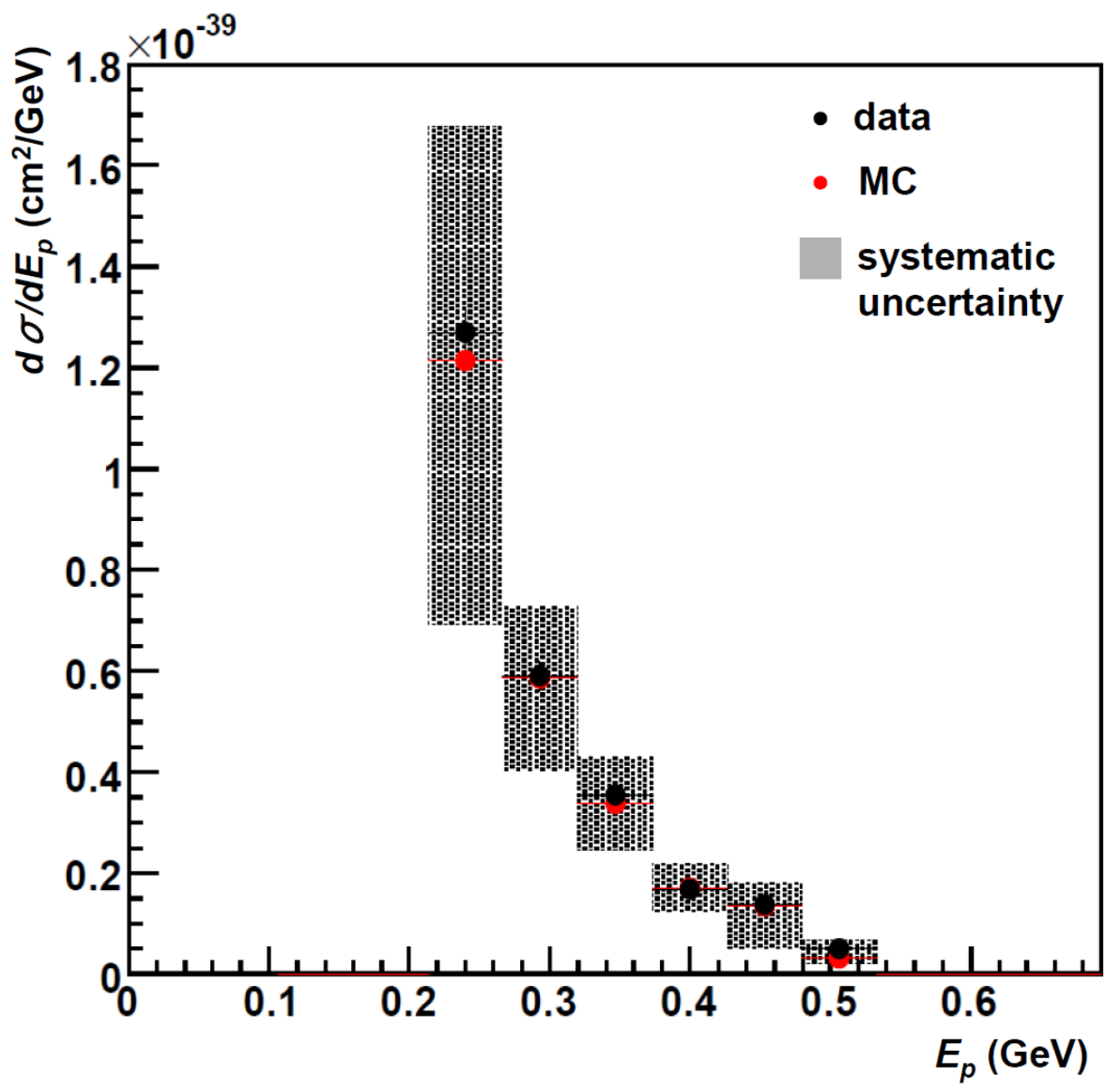

Figure 4.24: Total systematic uncertainty in NC elastic scattering cross section. 


\subsection{Nuclear Effect Correction}

$Q^{2}$ is calculated correctly from recoiled proton kinetic energy if the target proton is at rest in the initial state and does not interact with any nucleons. However, in the real case, the target protons have Fermi momentum. They are also bound with nuclear binding energy. Furthermore, they are occasionally rescattered by other nucleons in the same nucleus. Recoiled proton kinetic energy or scattering angle change due to these effects. These effects are called nuclear effects. It mainly comes from Fermi momentum in the nuclei. Since $Q^{2}$ is reconstructed from recoiled proton kinetic energy, $Q^{2}$ is smeared. Figure 4.25 shows a distribution of $Q^{2}$ reconstructed from proton kinetic energy v.s. $Q^{2}$ calculated from the neutrino momentum using Monte-Carlo simulation.
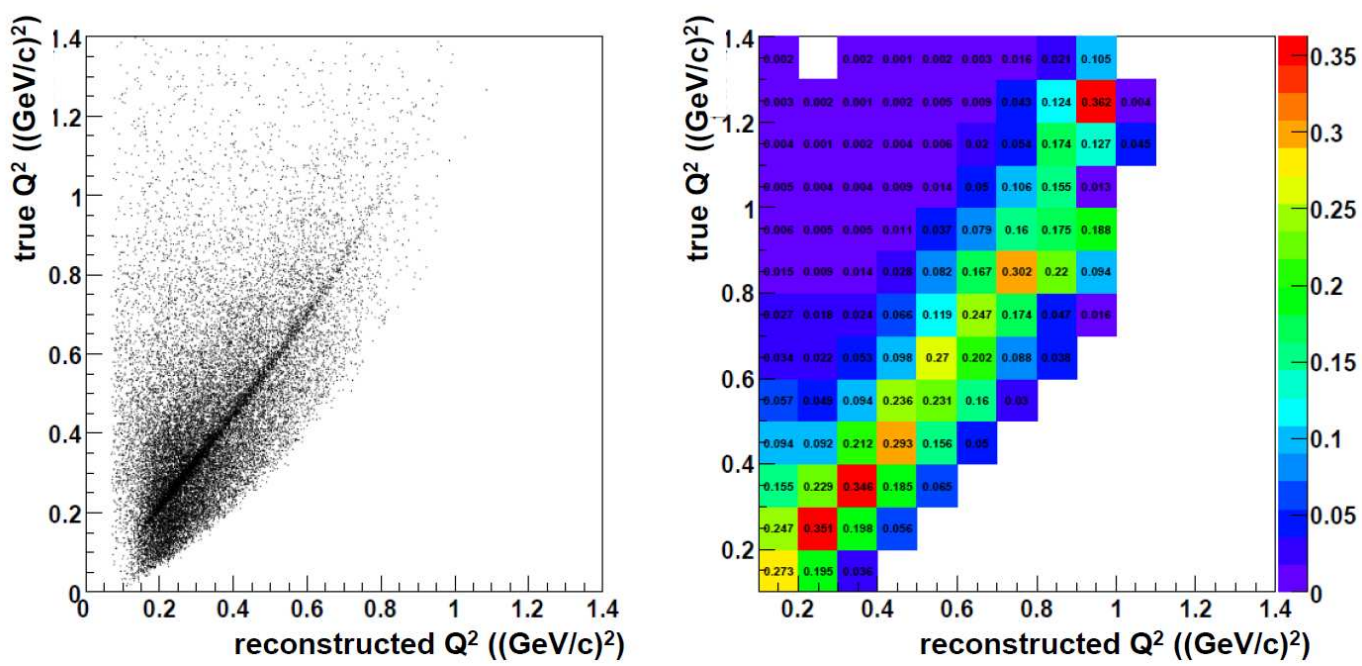

Figure 4.25: $Q^{2}$ reconstructed from proton kinetic energy (left) v.s. $Q^{2}$ calculated from the neutrino momentum using Monte-Carlo simulation (right).

Unsmearing matrix for nuclear correction $U_{n u c l}$ is made from this distribution. $U_{\text {nucl }}$ is defined as 


$$
U_{\text {nucl }}=\left(\begin{array}{ccccccccc}
0.27 & 0.20 & 0.04 & 0 & 0 & 0 & 0 & 0 & 0 \\
0.25 & 0.36 & 0.19 & 0.06 & 0 & 0 & 0 & 0 & 0 \\
0.16 & 0.23 & 0.35 & 0.19 & 0.09 & 0 & 0 & 0 & 0 \\
0.09 & 0.09 & 0.08 & 0.28 & 0.17 & 0.06 & 0 & 0 & 0 \\
0.07 & 0.05 & 0.06 & 0.24 & 0.23 & 0.18 & 0.03 & 0 & 0 \\
0.03 & 0.02 & 0.02 & 0.09 & 0.27 & 0.20 & 0.09 & 0.04 & 0 \\
0.03 & 0.02 & 0.01 & 0.04 & 0.12 & 0.25 & 0.17 & 0.05 & 0.02 \\
0.02 & 0.01 & 0.01 & 0.03 & 0.08 & 0.19 & 0.30 & 0.22 & 0.09 \\
0.01 & 0.01 & 0 & 0.01 & 0.04 & 0.08 & 0.18 & 0.18 & 0.19 \\
0 & 0 & 0 & 0.01 & 0.01 & 0.06 & 0.11 & 0.17 & 0.01 \\
0 & 0 & 0 & 0 & 0.01 & 0.02 & 0.06 & 0.17 & 0.13 \\
0 & 0 & 0 & 0 & 0.01 & 0.01 & 0.04 & 0.12 & 0.38
\end{array}\right)
$$

Reconstructed $Q^{2}$ can be corrected using unsmearing matrix. The same technique as the detector unfolding matrix is used. Figure 4.26 shows $Q^{2}$ distribution after nuclear effect correction.

Differential cross section as a function of $Q^{2}$ is evaluated using $Q^{2}$ distribution after nuclear effect correction. Differential cross section as a function of $Q^{2}$ is shown in figure 4.27. Error bars include statistical error only.

Since nuclear effects that generated in MC are used for nuclear effect correction, this $Q^{2}$ distribution is dependent on the model of the nuclear effects in MC. Study of the systematic uncertainty in $Q^{2}$ distribution after nuclear effect correction must include the systematic uncertainty in the model of nuclear effects in MC. 


\section{$Q^{2}$ (after nuclear effect correction)}

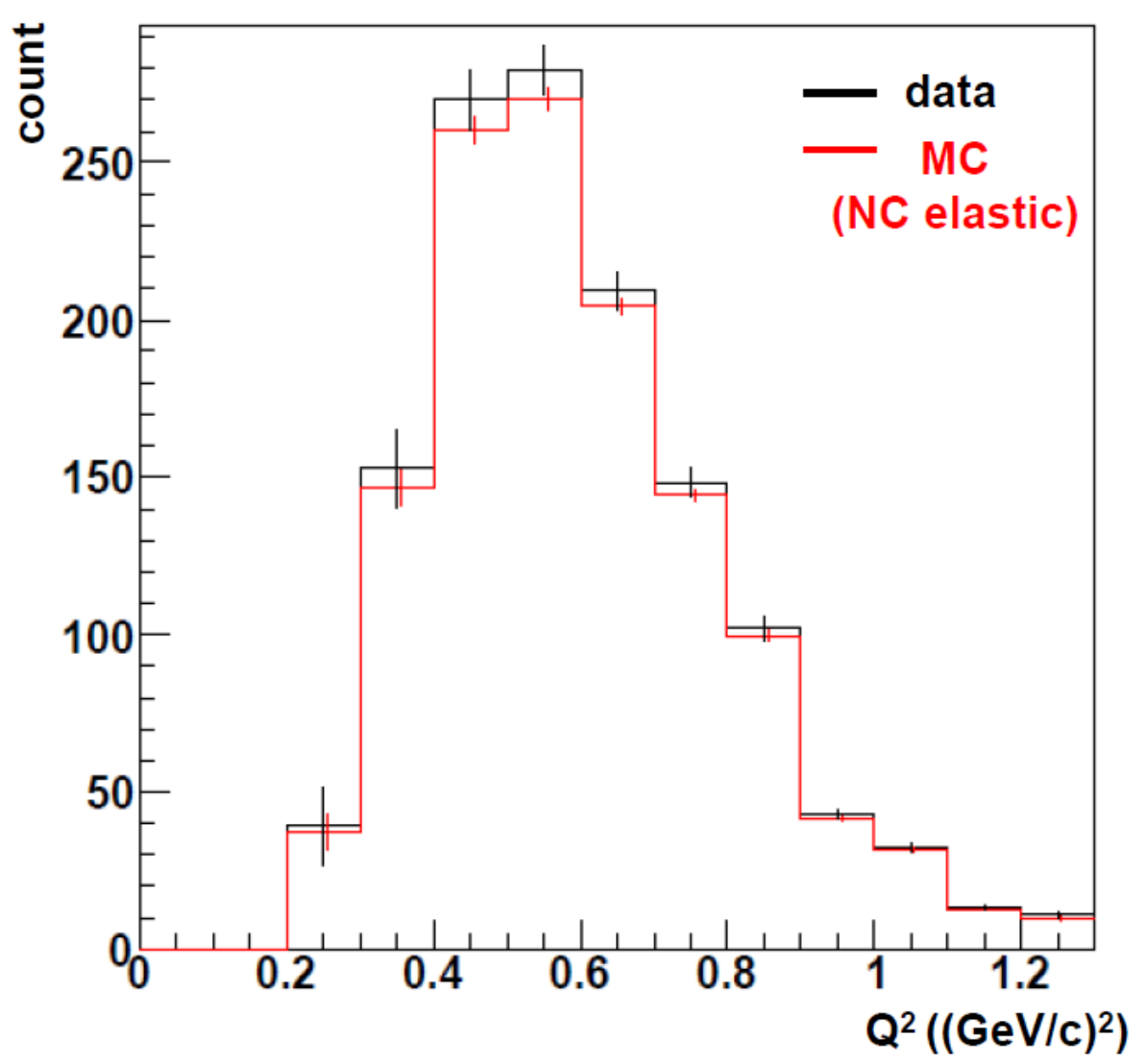

Figure 4.26: $Q^{2}$ distribution after nuclear effect correction. Data are compared to $\mathrm{MC}$ simulation. 


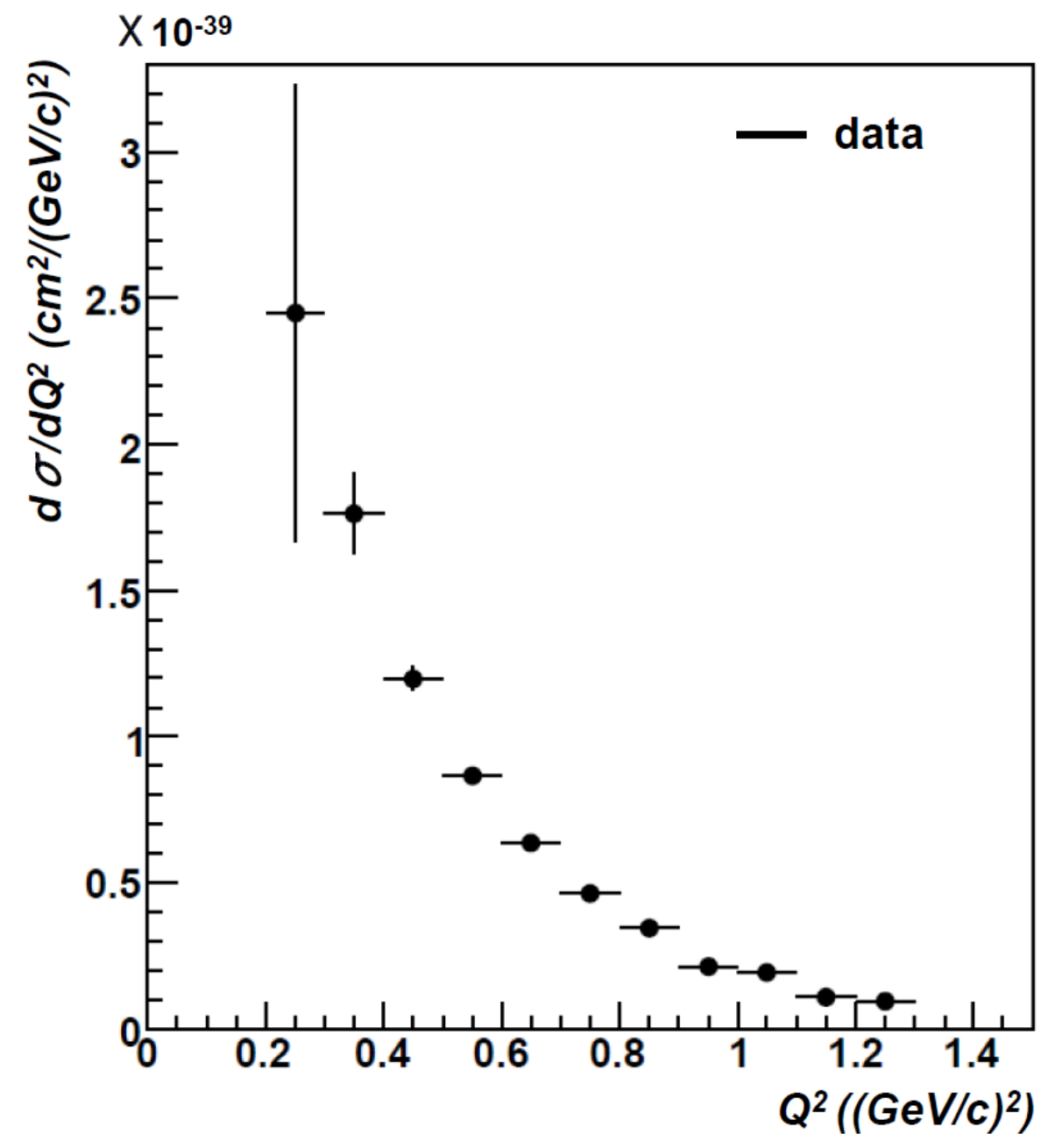

Figure 4.27: Differential cross section as a function of $Q^{2}$. Error bars include statistical error only. 



\section{Chapter 5}

\section{Result}

Differential cross section as a function of proton kinetic energy and $Q^{2}$ is measured using NC elastic data sample taken in SciBar. Signature of this event is single proton track. 8441 events in total are selected from all neutrino mode run. Those data correspond to $9.9 \times 10^{19}$ POT. Number of events in MC simulation is normalized by number of SciBar-MRD matched tracks. SciBar-MRD matched tracks are candidates of muons from $\mathrm{CC}$ reactions inside SciBar.

NC elastic data sample includes backgrounds mainly from NC pion production and dirt events. Those backgrounds are estimated and subtracted using the MC simulation. After the background subtraction, proton kinetic energy distribution of pure NC elastic data sample is obtained. Since proton kinetic energy distribution is biased by tracking resolution and detection efficiency, some corrections is performed to obtain unfolded distribution. $Q^{2}$ is reconstructed using proton kinetic energy. Nuclear effect correction need to be done to obtain the true $Q^{2}$ distribution by comparing true $Q^{2}$ and reconstructed $Q^{2}$ using MC. However, nuclear effect is dependent on the nuclear model in MC. First, NC elastic cross section as a function of proton kinetic energy is evaluated as the model independent result. Differential cross section is evaluated using proton kinetic energy distribution after detector unfolding and efficiency correction. Integrated flux is determined to be $1.91 \times 10^{-8}$ $\left(1 /\left(\mathrm{cm}^{2} \cdot \mathrm{POT}\right)\right)$ using beam MC simulation. Systematic uncertainty in $\mathrm{NC}$ elastic scattering cross section is estimated from uncertainties in neutrino beam, neutrino interaction and detector response.

Figure 5.1 shows total systematic uncertainty in NC elastic scattering cross section as a function of proton kinetic energy.

Differential cross section $d \sigma / d Q^{2}$ is evaluated using $Q^{2}$ distribution after nuclear effect correction.

Figure 5.2 shows differential cross section evaluated from data. Error bars 


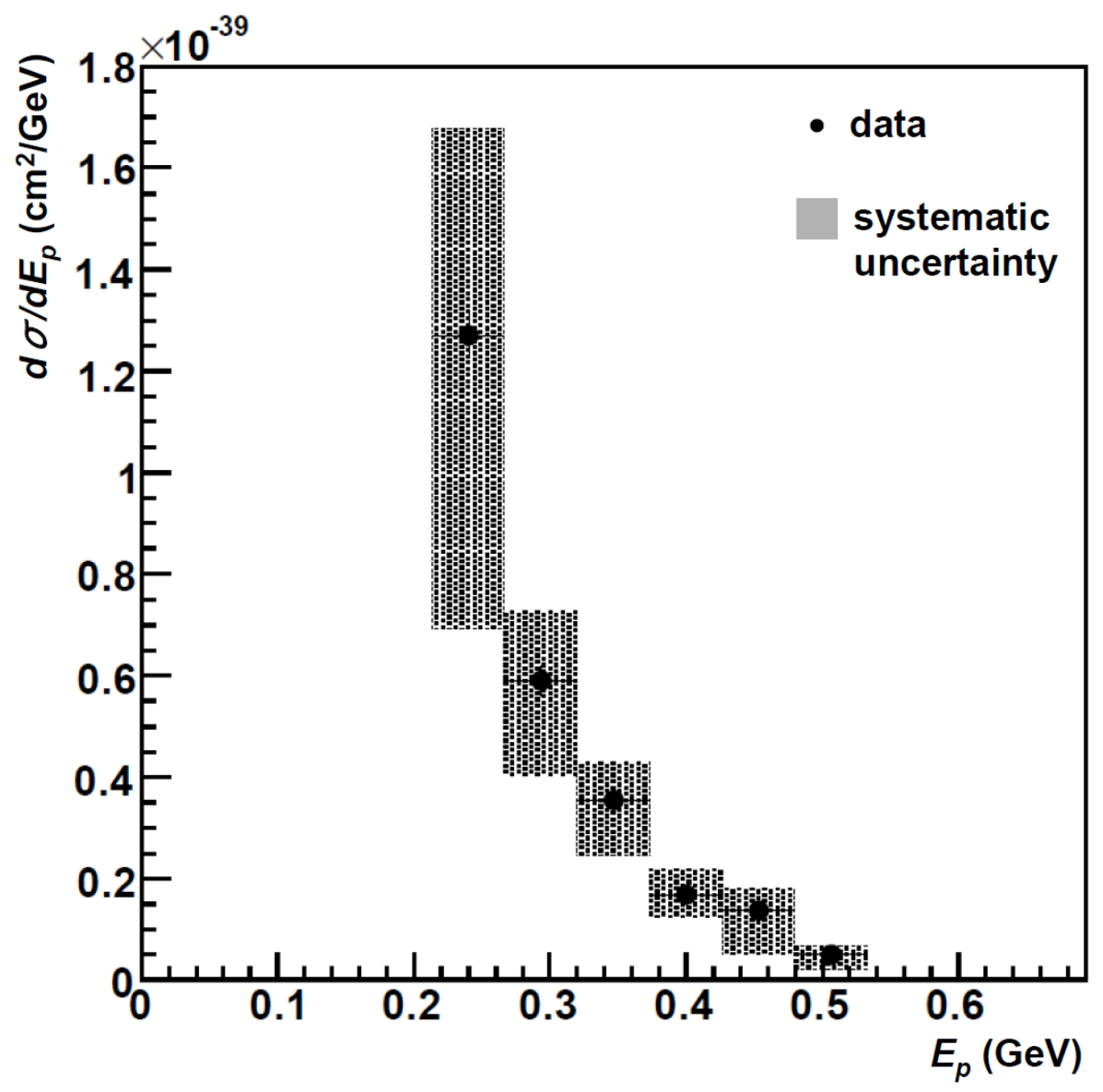

Figure 5.1: Total systematic uncertainty in NC elastic scattering cross section. 
include statistical error only. Systematic errors will be evaluated. Figure 5.3 shows differential cross section with the prediction from the MC simulation.

Figure 5.4 shows differential cross section compared with the experimental data from BNL E734 and MiniBooNE experiments. Error bars of E734 and MiniBooNE data include statistical and systematic errors. BNL E734 data shows the cross section for $\nu p \rightarrow \nu p$ scattering while SciBooNE and MiniBooNE data show the cross section per nucleon $(\nu N \rightarrow \nu N)$. Since MiniBooNE uses the same neutrino beam, it can be compared directly to the SciBooNE result. The result is in good agreement.

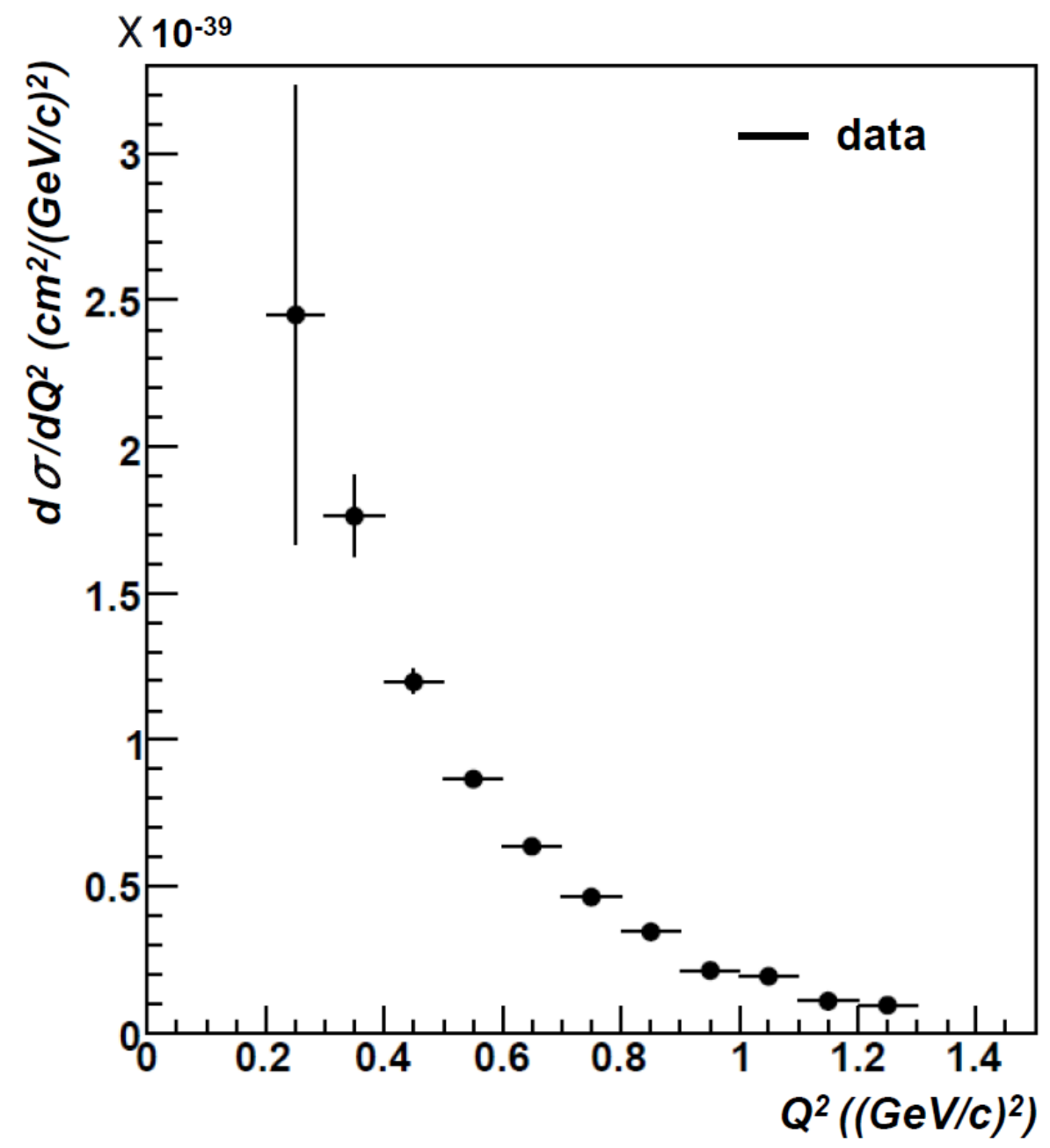

Figure 5.2: Differential cross section as a function of $Q^{2}$. Error bars include statistical error only. 


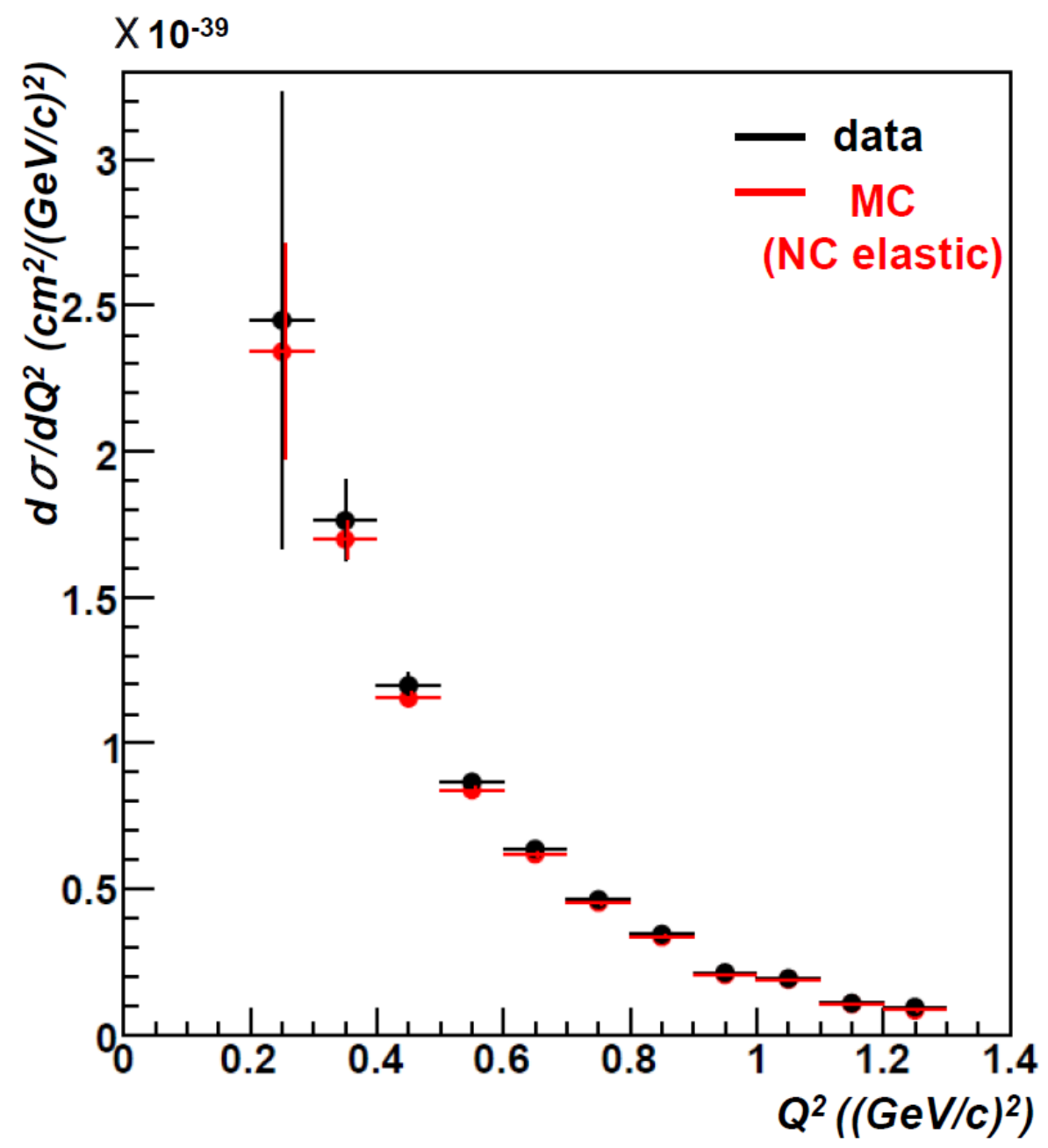

Figure 5.3: Differential cross section after corrections. Cross section from data is compared with MC simulation. MC plot is from NC elastic sample and processed in the same way as data. Error bars include statistical error only. 


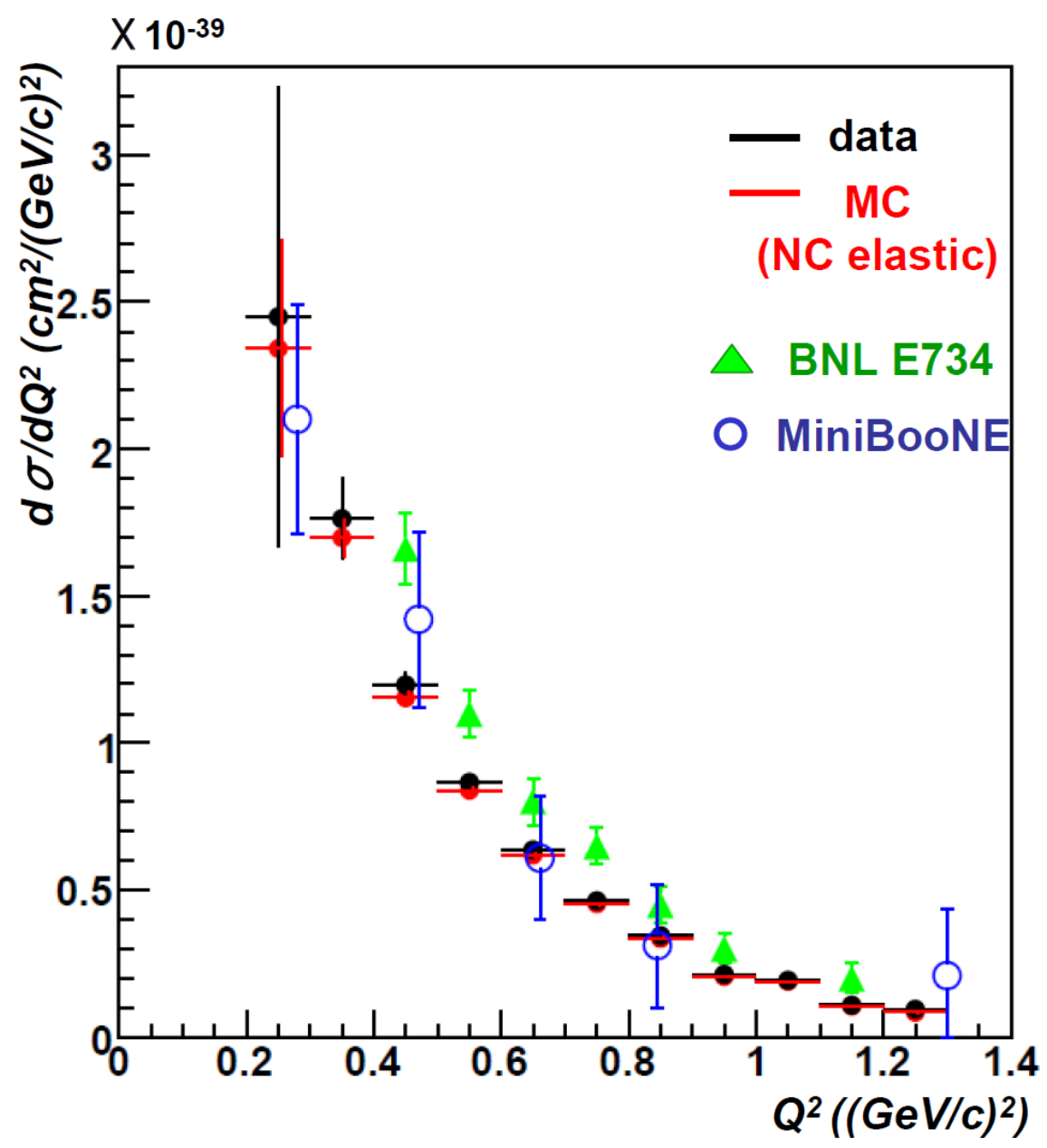

Figure 5.4: Figure 5.4 shows differential cross section compared with the experimental data from BNL E734 and MiniBooNE experiments. Error bars of E734 and MiniBooNE data include statistical and systematic errors. BNL E734 data shows the cross section for $\nu p \rightarrow \nu p$ scattering while SciBooNE and MiniBooNE data show the cross section per nucleon $(\nu N \rightarrow \nu N)$. 



\section{Chapter 6}

\section{Conclusion}

Cross section for $\mathrm{NC}$ elastic scattering is measured as a function of proton kinetic energy and $Q^{2}$. Data taken by SciBooNE detector in neutrino mode with POT of $9.9 \times 10^{19}$ are used.

Signal of NC elastic scattering is a single proton track. In $\nu p \rightarrow \nu p$ process, the recoil proton is detected. On the other hand, most of $\nu n \rightarrow \nu n$ is invisible because there are only neutral particles in final state, but sometimes recoil neutron is scattered by proton and recoil proton is detected. Signal of this event is also single proton track. Geometrical information is used for the event selection for the single track contained in SciBar fiducial volume. $d E / d x$ information of reconstructed track is used for proton identification.

After the event selection, NC elastic scattering sample which includes $\nu p \rightarrow \nu p$ and $\nu n \rightarrow \nu n$ is obtained.

The summary is:

- Neutrino is a clean probe to study nucleon and nuclear structure as it interacts only with weak force. The cross-section for neutrino-nucleon $\mathrm{NC}$ elastic scattering was measured in $Q^{2}>0.4 \mathrm{GeV}^{2}$ region by E734 experiment at BNL. Result from this experiment was the only published data for NC elastic scattering cross-section before our experiment.

- SciBooNE is an experiment for the measurement of neutrino-nucleon scattering cross-section in the region of the neutrino energy below 1 $\mathrm{GeV}$. In this energy region, the fraction of $\mathrm{NC}$ elastic scattering is evaluated to be $17.6 \%$ of total neutrino interaction.

- Signature of NC elastic scattering is single proton track. Event selection using geometrical information, multiple TDC hits, the stopping range 
and $d E / d x$ information was performed.

- 8441 events in total are selected from all neutrino mode run. Those data correspond to $9.9 \times 10^{19}$ POT.

- Major backgrounds are NC pion production and dirt neutrons. Dirt neutrons are neutrons recoiled by neutrino beam inside the walls of experimental hall or dirt around the detectors. Those backgrounds were estimated using MC simulation.

- After the proton kinetic energy distribution of pure NC elastic data sample was obtained, detector unfolding and efficiency correction were performed. These corrections are for the proton kinetic energy distribution smeared by tracking resolution and biased by detection efficiency.

- Differential cross-section was evaluated using unfolded proton kinetic energy distribution, the number of neutrinos that entered the detector, and the number of nucleon target. The number of neutrinos is determined using the beam MC simulation.

- Systematic uncertainty in NC elastic scattering cross section comes from uncertainties in neutrino beam, neutrino interaction and detector response.

- Nuclear effect correction was performed to obtain $Q^{2}$ distribution.

- Differential cross-section was compared with the results of previous NC elastic scattering cross-section measurement from BNL E734 and MiniBooNE. The SciBooNE result agrees well with them.

This thesis presents differential cross-section measurement of NC elastic scattering at SciBooNE. The result agrees well with previous NC elastic scattering cross-section measurement from BNL E734 and MiniBooNE. Further analysis such as detailed estimation of the nuclear effects are in the scope as a next stage of analysis. 


\section{Acknowledgement}

I would like to express my gratitude for Professor Toshi-Aki Shibata who has been my supervisor. He provided me the opportunities of working on my research. He has continuously encouraged, advised and supported me at every stage of this work. His advice through my thesiswork was essential for me to accomplish my research.

I am grateful to all members of the SciBooNE collaboration. I especially wish to acknowledge the spokespersons - Dr. Tsuyoshi Nakaya and Dr. Morgan Wascko -, the project manager - Dr. Richard Tesarek -, the analysis coordinators - Dr. Michel Sorel and Dr. Hidekazu Tanaka -, run coordinators - Dr. Masashi Yokoyama and Dr. Hidekazu Tanaka -, beam coordinator - Dr. Tom Kobilarcik -, detector coordinators - Dr. Hidekazu Tanaka, Dr. Lucio Ludovici and Dr. Robert Napora -, DAQ coordinator - Dr. Masashi Yokoyama -, and offline software coordinator - Dr. Michel Sorel. They organized all operations necessary for experiments and analyses, and supported my activities. I also would like to thank all peoples who shared really good time at FNAL and who discussed with me about physics, my analysis and everything else.

I thank all members of Shibata Lab. at Tokyo Institute of Technology. Especially, Dr. Yoshiyuki Miyachi, the assistant professor, helped me in my analysis and computing. Mr. Yoshimitsu Imazu, Dr. Xiaorui Lu and Mr. Kouichi Sakashita discussed with me about physics.

I appreciate all smooth and heartful supports by Ms. Akiko Okawa and Ms. Ami Saito as the secretaries at Tokyo Institute of Technology, Ms. Misato Kodaka as the secretary at KEK, Ms. Kyoko Kunori and Ms. Crae Tate as the secretaries at FNAL. I was able to efficiently perform this work by the corporation of them. 



\section{Bibliography}

[1] L. A. Ahrens et al., Phys. Rev. D 35, 785 (1987).

[2] A. A. Aguilar-Arevalo, et al., hep-ex/0601022 (2006).

[3] F. J. Hasert et al., Phys. Lett. B 46:121 (1973).

[4] F. J. Hasert et al., Phys. Lett. B 46:138 (1973).

[5] W. Lee et al., Phys. Rev. Lett. 37:186 (1976).

[6] D. Cline et al., Phys. Rev. Lett. 37:252 (1976).

[7] D. Cline et al., Phys. Rev. Lett. 37:648 (1976).

[8] J. Horstkotte et al., Phys. Rev. D 25:2743 (1982).

[9] M. Bernardini et al., Phys. Lett. B 46, 261 (1973).

[10] G. T. Garvey, W. C. Louis, and D. H. White, Phys. Rev. C 48, 761 (1993).

[11] Y. Itow, et al. hep-ex/0106019 (2001).

[12] A. A. Aguilar-Arevalo, et al. Phys. Rev. Lett. 98, 231801 (2007).

[13] The K2K Collaboration Phys. Rev. D 74, 072003 (2006).

[14] L.A.Ahrens et al. Phys. Rev. D35, 785 (1987).

[15] Nitta K et al. Nucl. Instrum. Meth. A535, p147 (2004).

[16] C. Giganti AIP Conf. Proc. 967, p301 (2007).

[17] J. Walding AIP Conf. Proc. 967, p289 (2007).

[18] S. Buontempo et al. Nucl. Instrum. Meth. A349 (1994). 
[19] S. Agostinelli et al. [GEANT4 Collaboration], Nucl. Instrum. Meth. A 506, 250 (2003).

[20] A. A. Aguilar-Arevalo et al. [MiniBooNE Collaboration], arXiv:0806.1449[hep-ex].

[21] M. G. Catanesi et al., Eur. Phys. J. C 52, 29 (2007) [arXiv:hepex/0702024].

[22] I. Chemakin et al. [E910 Collaboration], Phys. Rev. C 77, 015209 (2008) [Erratum-ibid. C 77, 049903 (2008)] [arXiv:0707.2375[nucl-ex]].

[23] J. R. Sanford and C. L. Wang, BNL AGS Internal Report p. 11299 (1967).

[24] M. H. Shaevits, Determination of The $K^{+}$Production Cross Section at 8 GeV Using External Data, BooNE Technical Note 209 (2006).

[25] A. Aleshin et al., ITEP-77-80 (1977).

[26] J. V. Allaby et al., CERN 70-12, Nuclear Physics Division (1970).

[27] D. Dekkers et al., Phys. Rev. 137:962 (1965).

[28] R. A. Lundy et al., Phys. Rev. Lett. 14:504 (1965).

[29] G. J. Marmer et al., Phys. Rev. 179 (1969).

[30] I. A. Vorontsov et al., ITEP-88-11 (1988).

[31] [E-802 collaboration], T. Abbott et al., Phys. Rev. D 45:3906 (1992).

[32] P. A. Piroue et al., Phys. Rev. 148 (1966).

[33] T. Eichten et al., Nucl. Phys. B 44 (1972).

[34] Y. Hayato, Nucl. Phys. Proc. Suppl. 112, 171 (2002).

[35] G. Mitsuka, AIP Conf. Proc. 981, 262 (2008).

[36] C. H. Llewellyn Smith, Phys. Rept. 3, 261 (1972).

[37] R. A. Smith and E. J. Moniz, Nucl. Phys. B 43, 605 (1972) [Erratumibid. B 101, 547 (1975)].

[38] D. Rein and L. M. Sehgal, Annals Phys. 133, 79 (1981). 
[39] C. Berger and L. M. Sehgal, Phys. Rev. D 76, 113004 (2007) [arXiv:0709.4378[hep-ph]].

[40] K. S. Kuzmin, V. V. Lyubushkin and V. A. Naumov, Mod. Phys. Lett. A 19, 2815 (2004) [Phys. Part. Nucl. 35, S133(2004)[arXiv:hep$\mathrm{ph} / 0312107]]$.

[41] D. Rein, Z. Phys. C 35, 43 (1987).

[42] T. Kitagaki et al. [KAMIOKANDE Collaboration], J. Phys. Soc. Jap. $\mathbf{5 5}, 3786$ (1986).

[43] S. K. Singh, M. J. Vicente-Vacas and E. Oset, Phys. Lett. B 416, 23 (1998) [Erratum-ibid. B 423, 428 (1998)].

[44] D. Rein and L. M. Sehgal, Nucl. Phys. B 233, 29-44 (1983).

[45] M. Hasegawa et al., Phys. Rev. Lett. 95, 252301 (2005).

[46] D. Rein and L. M. Sehgal, hep-ph p.0606185 (2006).

[47] A. Kartavtsev, E. A. Paschos, and G. J. Gounaris, Phys. Rev. D 74, 054007 (2006).

[48] H. W. Bertini, Phys. Rev. C 6, 631 (1972).

[49] S. J. Lindenbaum, Phys. Rev. 105, 1874 (1957).

[50] V. Ammosov for the SKAT Collaboration, NuInt01 Workshop at KEK, Tsukuba, Japan (2001).

[51] A. Heikkinen, N. Stepanov and J. P. Wellisch, In the Proceedings of 2003 Conference for Computing in High-Energy and Nuclear Physics (CHEP03), La Jolla, California, 24-28 Mar 2003, pp MOMT008 [arXiv:nucl-th/0306008].

[52] H. W. Bertini, Phys. Rev. 131, 1801 (1963); 138, AB2 (1965).

[53] H. W. Bertini, Phys. Rev. 188, 1711 (1969).

[54] D. V. Bugg et al., Phys. Rev. 146, 980 (1966).

[55] U. E. Kruse et al., Phys. Rev. 103, 211 (1956).

[56] T. Ferbel et al., Proceedings of the 1962 International Conference on High-Energy Physics at CERN, p.76 (1962). 
[57] S. P. Kruchinin et al., Yadern. Fiz. 1, 317 (1965).

[58] J. H. Atkinson et al., Phys. Rev. 123, 1850 (1961).

[59] P. H. Bowan et al., Nucl. Phys. 22, 640 (1961).

[60] S. Focadi et al., Nuovo Cimento 39, 405 (1965).

[61] A. F. Donaitsev et al., Zh. Eksperim. i Teor. Fiz. 36, 1656 (1959).

[62] J. G. Rushbrooke et al., Nuovo Cimento 33, 1509 (1964).

[63] Yu. D. Bayukov et al., Nucl. Phys. 4, 61 (1957).

[64] J. Birks, Theory and Practice of Scintillation Counting, Pergamon Press, 1964.

[65] M. Hasegawa, Ph.D. thesis, Kyoto University, 2006.

[66] H. Maesaka, Ph.D. thesis, Kyoto University (2005).

[67] Y. Kurimoto, NOW2008 Workshop at Otranto, Leece, Italy (2008).

[68] R. Gran et al., [K2K Collaboration], Phys. Rev. D 74, 052002 (2008) [arXiv:hep-ex/0603034].

[69] A. A. Aguilar-Arevalo et al., [MiniBooNE Collaboration], Phys. Rev. Lett. 100, 032301 (2008) [arXiv:0706.0926].

[70] V. Bernard, L. Elouadrhiri, and U. G. Meissner, J. Phys. G 28, R1-R35 (2002) [arXiv:hep-ph/0107088]. 